\title{
Review
}

Wei Shi*, Ye Tian and Antoine Gervais

\section{Scaling capacity of fiber-optic transmission systems via silicon photonics}

https://doi.org/10.1515/nanoph-2020-0309

Received May 31, 2020; accepted September 21, 2020;

published online October 22, 2020

Abstract: The tremendous growth of data traffic has spurred a rapid evolution of optical communications for a higher data transmission capacity. Next-generation fiberoptic communication systems will require dramatically increased complexity that cannot be obtained using discrete components. In this context, silicon photonics is quickly maturing. Capable of manipulating electrons and photons on the same platform, this disruptive technology promises to cram more complexity on a single chip, leading to orders-of-magnitude reduction of integrated photonic systems in size, energy, and cost. This paper provides a system perspective and reviews recent progress in silicon photonics probing all dimensions of light to scale the capacity of fiber-optic networks toward terabits-per-second per optical interface and petabits-per-second per transmission link. Firstly, we overview fundamentals and the evolving trends of silicon photonic fabrication process. Then, we focus on recent progress in silicon coherent optical transceivers. Further scaling the system capacity requires multiplexing techniques in all the dimensions of light: wavelength, polarization, and space, for which we have seen impressive demonstrations of on-chip functionalities such as polarization diversity circuits and wavelength- and space-division multiplexers. Despite these advances, large-scale silicon photonic integrated circuits incorporating a variety of active and passive functionalities still face considerable challenges, many of which will eventually be addressed as the technology continues evolving with the entire ecosystem at a fast pace.

*Corresponding author: Wei Shi, Université Laval, Québec, QC, Canada, E-mail: wei.shi@gel.ulaval.ca. https://orcid.org/00000001-5781-9912

Ye Tian and Antoine Gervais, Centre d'optique, photonique et laser (COPL) and the Département de génie électrique et génie informatique, Université Laval, Québec, QC G1V 0A6, Canada, E-mail: ye.tian.2@ulaval.ca (Y.Tian), antoine.gervais.1@ulaval.ca (A. Gervais). https://orcid.org/0000-0001-7210-152X (Y. Tian). https://orcid.org/0000-0002-3704-1303 (A. Gervais)
Keywords: fiber-optic transport networks; high-capacity optical transmissions; nanophotonic devices; optical communications; optical multiplexing; optical transceivers; photonic integrated circuits; silicon photonics.

\section{Introduction}

Global Internet traffic has been growing exponentially over the past 20 years [1-4]. Anticipating a 40\% compound annual growth rate in data demand, a "capacity crunch" in fiber-optic transmission that could severely constrain the Internet growth was predicted for around 2020 [2]. The actual growth of Internet traffic was at a lower rate over the last few years (20-30\%) [3] but shows a spike in 2020 due to the COVID-19 pandemic that has caused a significant traffic increase during the lockdown period [5]. The amount of bytes flowing around the world will continue to scale up fast in the era of Internet of Things and 5G. It is predicted that the number of devices connected to networks will be more than three times the global population by 2023 [6].

The tremendous growth of Internet traffic has spurred a rapid evolution of optical communication technology for higher data transmission capacity, as shown in Figure 1. Digital signal processing (DSP) enabled coherent optical communications with advanced modulation formats [7], which led to giant leaps in single-channel data rates from 10 to $400 \mathrm{~Gb} / \mathrm{s}$ in optical transport networks [8]. Leading system vendors have recently announced commercial solutions for $600 \mathrm{~Gb} / \mathrm{s}$ across $1000 \mathrm{~km}$ and $800 \mathrm{~Gb} / \mathrm{s}$ for shorter reach distances. Next-generation fiber-optic communication systems exceeding 1 terabits-per-second $(\mathrm{Tb} / \mathrm{s})$ per wavelength will require dramatically increased complexity that cannot be obtained using discrete optical components. InP-based $500 \mathrm{~Gb} / \mathrm{s}$ coherent transceivers already contain hundreds of optical components and connectors [9]. An ultrahigh-capacity transmission system exceeding petabits-per-second $(\mathrm{Pb} / \mathrm{s})$ will comprise a vast number of wavelength-spatial channels on the 10,000 scale (e.g., 100+ wavelengths times 10+ spatial paths; see Figure 1). Due to this unprecedented complexity, optical transceivers dominate the cost of coherent transmission 
systems. Photonic integrated circuits (PICs), especially on the silicon platform, provide a promising solution to resolve the dilemma of scaling system complexity while reducing cost per bit.

Along with the explosion of data traffic, silicon photonics has quickly emerged as an enabling technology to drastically reduce the energy consumption and cost of optical communications systems [21-26]. Capable of manipulating electrons and photons on the same platform, silicon photonics promises to cram more complexity on a single chip. It uses the advanced manufacturing process of microprocessors, known as the complementary metaloxide-semiconductor (CMOS) process, where tens of billions of electronic components have been integrated on a single chip. This disruptive technology is based on highindex-contrast materials allowing for extremely strong optical confinement on the nanometer scale, leading to orders-of-magnitude reduction in size, energy, and cost. Furthermore, silicon photonic integrated circuits (Si-PICs) can be readily integrated with advanced electronics. These advantages make the technology immensely appealing to a vast number of applications, such as portable sensors [27], quantum computing [28], and artificial intelligence [29]. In particular, large-scale silicon PICs are essential to the capacity scaling of future optical transmission systems.

The capacity of a fiber-optic transmission link $C$ can be described as follows [1]

$$
C=2 M B \log _{2}(1+\mathrm{SNR})
$$

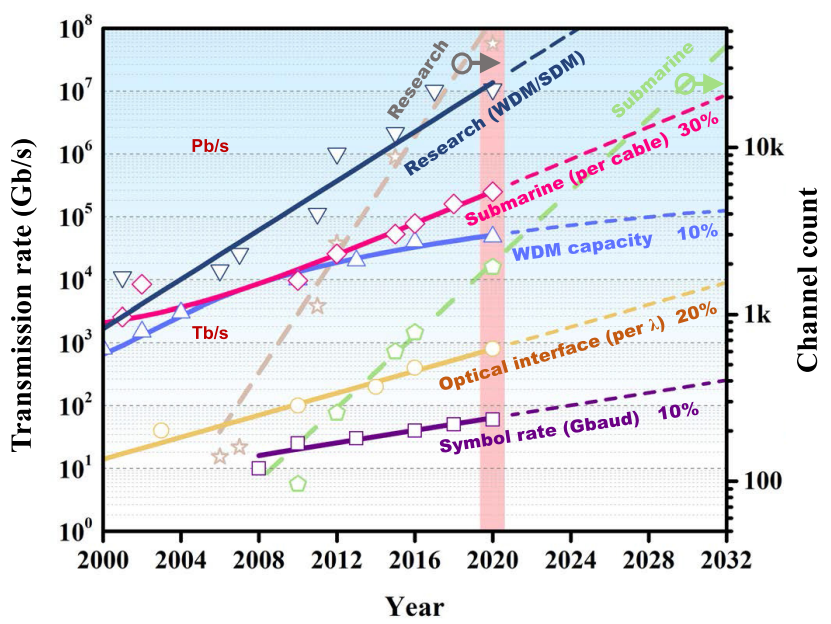

Figure 1: Evolution of fiber-optic transmission capacity. The channel counts are estimated based on the product of amounts of wavelengths and spatial paths. The data of commercial systems (including symbol rate, optical interface, WDM capacity and submarine systems) are extracted from $[1,10]$ for 2000-2018 and from $[11,12]$ for 2019-2020. The lab research results are extracted from [13-20]. WDM, wavelengthdivision multiplexing; SDM, space-division multiplexing; $\lambda$, wavelength. where $M, B$, and SNR represent the number of spatial paths, the total system bandwidth, and the signal-to-noise ratio (i.e., the ratio of average received signal power over the bandwidth to the average power of the noise and interference over the bandwidth), respectively. Apparently, this equation is a simple expansion of the classic ShannonHartley theorem [30] taking into consideration the multiplexing techniques in various physical dimensions: polarization, optical frequency (wavelength), and space.

The scaling of the overall transmission capacity relies on advances in these following aspects (Figure 2):

- Spectral efficiency: Leapfrogging in spectral efficiency (SE) and single-carrier transmission rate has been achieved using coherent detection with higher-order modulation formats, such as quadrature phase-shift keying (QPSK) and higher-order quadrature amplitude modulation (QAM) enabled by advanced DSP to compensate for impairments of the transmission link [31, 32]. Digital pulse shaping in time domain has pushed the SE to the Nyquist limit for intersymbol interference-free transmission [33]. However, due to the logarithmic (sublinear) scaling law (Eq. (1)), only marginal gain in capacity can be achieved by further increasing SNR [34]. For example, in a recent transmission experiment using a silicon modulator, an optical signal-to-noise ratio (OSNR) improvement of 4-dB (i.e., 2.5 times) allows to push the modulation from 16-QAM to 32-QAM at 60 GBaud, indicating an enhancement of $25 \%$ in SE [35]. Recent advances in constellation shaping techniques have achieved a tiny gap of down to $0.1 \mathrm{~dB}$ from the Shannon limit [36]. Therefore, further scaling the capacity of optical transmission systems relies on massive parallelism in the space-division multiplexing $(\mathrm{SDM}) \times$ wavelengthdivision multiplexing (WDM) space.

- Polarization-division multiplexing: Exploriting two orthogonal states of polarization doubles the transmission capacity. Compact, broadband, and highperformance polarization splitters/combiners and rotators can be readily implemented on the submicrometer silicon platform and monolithically integrated with coherent transceivers for polarizationdivision multiplexing (PDM), which has been a remarkable advantage compared to InP.

- Wavelength-division multiplexing: The total spectral resource sets the upper bound of the transmission capacity via a single-mode fiber. It is usually determined by the limited bandwidth of optical amplifiers placed periodically along the transmission link. Erbium-doped fiber amplifiers typically cover the $C$ band (1530-1565 nm) and can also be extended to the $L$ 


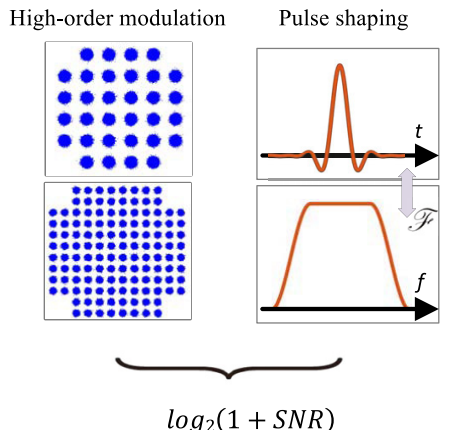

- IQ modulator

- Balanced PD

- Optical hybrid

- Thermo-optic phase shifter

- Tap PD monitor

- Broadband coupler/splitter

- CMOS ASIC (DSP)

(a)

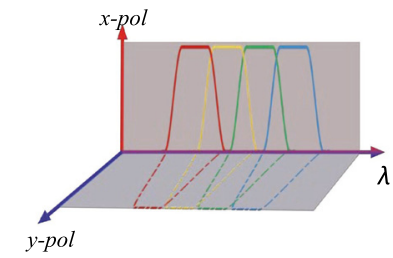

PDM (capacity $\times 2$ )

- Polarization splitter/combiner

- Polarization rotator

- 2D grating couplers or emitters

(b)

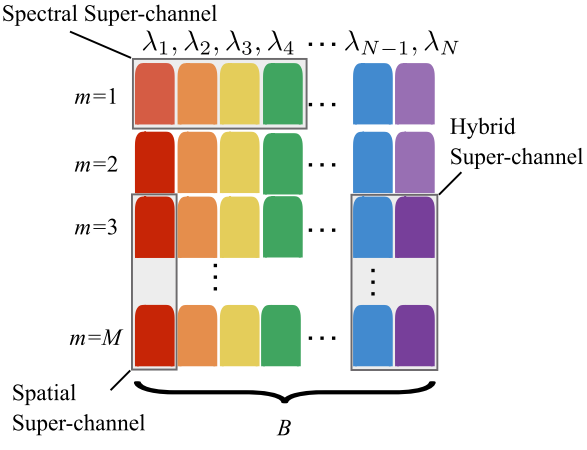

WDM $\times$ SDM

- Optical filters: AWG, MRR, MZI, etc.

- Thermo-optic phase shifter

- 2D/3D optical interface for mode conversion

- Varied optical attenuator

- Waveguide crossing

(c)

Figure 2: Scaling the transmission capacity of an optical link in various dimensions, following Eq. (1), including (a) augmenting the spectral efficiency through higher modulation formats with coherent detection (quadrature) and pulse shaping (time); (b) capacity doubling by PDM (polarization); (c) WDM $\times$ SDM (frequency and space). The required PIC building blocks are listed under respective functions. WDM, wavelengthdivision multiplexing; SDM, space-division multiplexing; PDM, polarization-division multiplexing; PIC, photonic integrated circuit.

band (1565-1625 nm). $C+L$ transpacific transmission systems have been recently deployed [37]. Recent advances in optical amplifiers have enabled demonstration of ultrawide-band transmissions [38-40]. Compatibility with ultrawide-band operation will be essential for integrated photonic devices and circuits.

- Space-division multiplexing: Space is the only remaining dimension that promises a long-term scaling of system capacity by orders of magnitude [1]. Parallel single-mode fibers (SMFs) have been used for point-to-point links such as submarine cables and data center interconnects, which are regarded as the first-generation SDM systems [37]. As SMFs are approaching their fundamental limits, SDM has become the new research frontier of optical multiplexing techniques with the emergence of new types of optical fibers. Large-scale integration of photonic functions such as arrays of in-phase/quadrature (IQ) modulators and coherent receivers will become crucial for the evolution of optical transmission systems toward massive parallelism.

An envisioned SDM-WDM transceiver system is shown in Figure 3. Assuming each of the transceivers delivers $1 \mathrm{~Tb} / \mathrm{s}$, a $1 \mathrm{~Pb} / \mathrm{s}$ system consisting of $1000 \mathrm{sub}$ channels (e.g., 100 wavelengths $\times 10$ spatial paths) will require 4000 modulators and 8000 photodetectors
(4 modulators and 4 balanced photodetectors for dualpolarization quadrature) and a large number of other active and passive components (e.g., wavelength and mode (de-)multiplexers, variable optical attenuators (VOAs), splitters/combiners, couplers, polarization splitters and rotators, etc.). All these components can be integrated on silicon chips, either monolithically or in a hybrid manner. Given the very limited gain possibly achieved by SNR improvement as discussed above, integration scalability is more critical than performance of individual functions. Silicon photonics provides an unbeatable platform to scale the complexity of integrated WDM $\times$ SDM systems.

In this paper, we review recent progress and challenges in silicon photonics, probing all dimensions of light to scale the capacity of fiber-optic transmission systems toward terabit per second and more $(\mathrm{Tb}+/ \mathrm{s})$ per optical interface and petabit per second and more $(\mathrm{Pb}+/ \mathrm{s})$ per transmission link. The rest of this paper is organized as follows. In Section 2, we overview fundamentals and the evolving trends of silicon photonic fabrication processes. Section 3 reviews recent progress in silicon photonic coherent transceivers. We will discuss challenges facing ultrahigh-speed transceivers exceeding $\mathrm{Tb} / \mathrm{s}$ and possible solutions. In Section 4, we review recent demonstrations of on-chip multiplexing functionalities in polarization, wavelength, and space, such as polarization circuits, and 


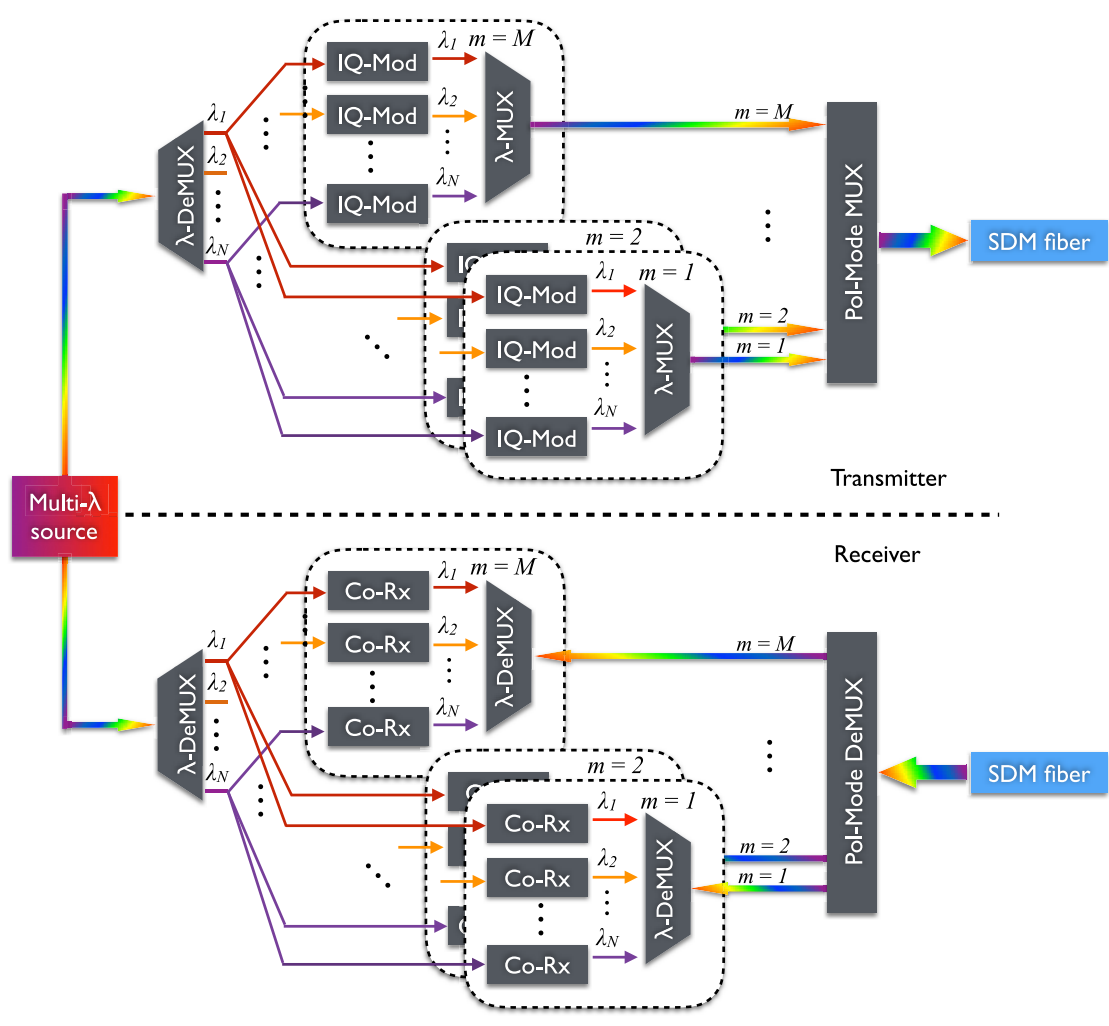

Figure 3: Schematic of an envisioned SDM-WDM transceiver system that supports $N$ wavelengths $\times M$ spatial paths in a fiber link. The arrows indicate the optical signal flows. For example, the transmission capacity can reach $1.2 \mathrm{~Pb} / \mathrm{s}$ using 120 wavelength (covering $C$ and $L$ bands) times 10 spacial modes at $1 \mathrm{~Tb} / \mathrm{s}$ per channel (e.g., 100 Gbaud DP-64QAM with a 20\% forward error correction (FEC) overhead). A multiwavelength laser system is used for both the input (at the transmitter side) and the local oscillators (at the receiver side). It can be either a high power optical frequency comb or an array of multiplexed single-mode lasers. Its power (including all the wavelengths) is split, equally in the ideal case, into $M$ spatial paths. Each transceiver array consists of $N \times$ IQ modulators (IQ-Mod) and coherent receivers (CoRXs). Pol-Mode (De)MUX, polarizationmode (de)multiplexer. wavelength- and space-division multiplexers. We conclude the diversity paper in Section 5.

\section{Overview of silicon nanophotonic fabrication}

In addition to the technological advantages, such as high-density photonic integration and cointegration with electronics, the economic promise has motivated the development of silicon photonics: low-cost per unit manufacturing can be achieved when chips are massively produced. This is enabled by the reuse of the mature CMOS processes (65-130 nm nodes), leveraging the huge capital expenditures in infrastructure and development already made by the microelectronics industry [41]. In this section, we give a brief overview of the silicon photonic material platforms, fabrication process, ecosystem, and future trends. We focus solely on material platforms that (1) support the optical communication windows $(\sim 1.3-1.6 \mu \mathrm{m}$ for current systems and may be extended to $2 \mu \mathrm{m}$ in the future) and (2) are CMOS compatible and supported by open-access foundries. Both the silicon-on-insulator (SOI) and silicon-nitride-on-insulator (SNOI) platforms respect these conditions. Other silicon-based or hybrid material platforms are in active development for UV [42] to the midIR applications $[43,44]$ but beyond the scope of this paper.

\subsection{Material platforms and CMOS- compatible processes}

Most recent research and development efforts in both academia and industry were made on the submicron SOI, where PICs are defined on a crystalline silicon thin film having a thickness usually well below micrometer. While other options are available, the most popular silicon thickness adopted by foundries is $220 \mathrm{~nm}$, even though some critics suggest that a thicker layer may lead to a better tradeoff between general performance metrics and fabrication tolerance [45]. Thanks to the large refractive index contrast between the silicon waveguide core $(\sim 3.5$ near $1550 \mathrm{~nm})$ and its oxide clad, the submicron SOI allows for low-loss passive strip and rib waveguides and very tight bends (below $10 \mu \mathrm{m}$ ) for a high device density. A diversity of passive components have been demonstrated for signals routing, filtering, mixing, (de)multiplexing, and polarization control [21, 46, 47] (Section 4). Efficient optical inputs/outputs for fiber coupling are available in the forms of grating couplers and edge couplers [48, 49]. The grating couplers provide a convenient means of high-tolerance vertical surface 

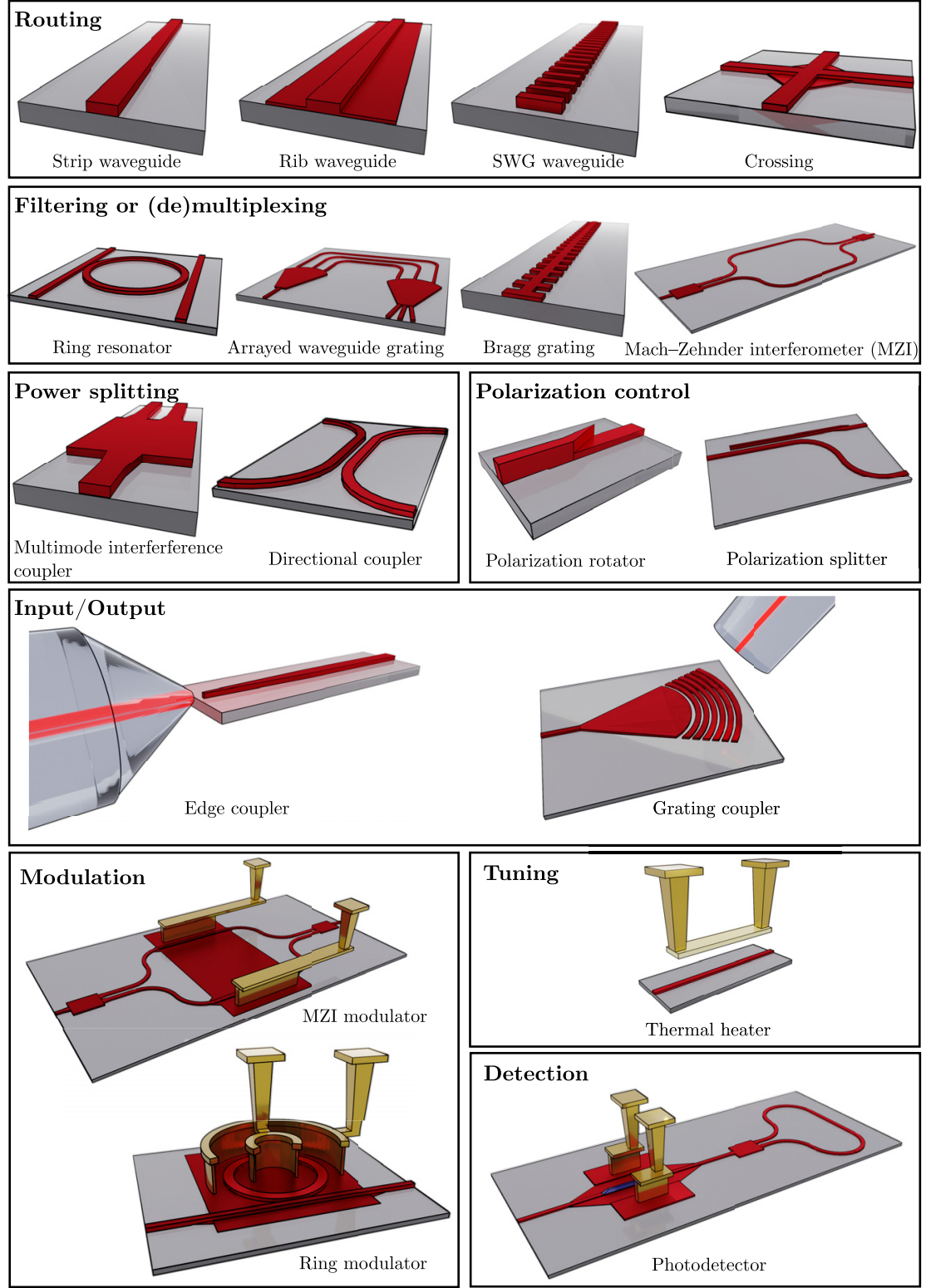

Figure 4: Mock-ups of the most common building blocks used in silicon photonics, classified by functionality. The structures are not to scale, and the top cladding layer is absent for visibility. The colors of the materials are the same as in Figure 5. coupling, enabling fast wafer-level test and 2D integration of optical interfaces [50]. Efficient edge coupling used to be a big challenge due to the large mode mismatch between a silicon waveguide and an optical fiber. This challenge has been well mitigated via techniques such as oxide cantilever with a silicon inverse taper [51], multilayer thin-film mode conversion [52], and subwavelength taper engineering [53]. Active devices such as high-speed modulators and photodetectors are available through CMOS-compatible doping process and Ge-on-Si epitaxy. The building blocks required to obtain those functionalities are shown in Figure 4.

These passive and active functionalities are implemented monolithically by multiple process steps on submicron SOI wafers (see Figure 5), usually including multi-level (partial and full) deep UV photolithography etch of silicon thin films, ion implantation for multi-level $p$-type and $n$-type doping, germanium deposition on silicon, etching and implantation for photodetectors, multilevel metals, oxide deposition, and deep trench for edge coupling. SOI platforms with a thicker silicon layer, usually a few micrometers (e.g., $3 \mu \mathrm{m}$ [54]), also exist for highpower applications, but they do not offer the high-speed active functionalities required for datacom and telecom applications.

The other integrated platform of interest is SNOI, as it provides complimentary functionalities and strengths [55, 

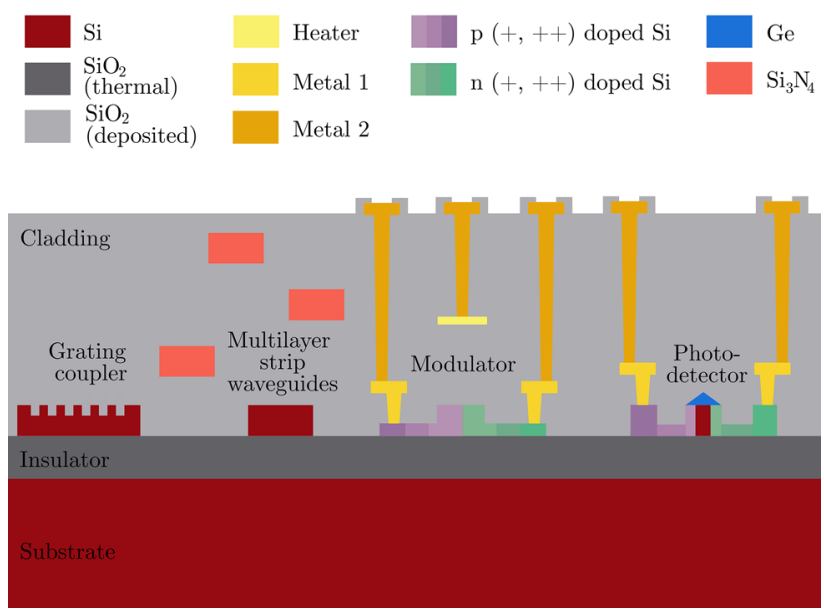

Figure 5: Schematic of the cross section of different integrated photonic devices fabricated with multilayer silicon nitride on submicron silicon-on-insulator process, comprising a grating coupler, strip waveguides, a modulator, and a photodetector (not to scale). It illustrates the different materials and process steps required during the fabrication.

56]. It was also demonstrated to be compatible with fabrication by deposition on top of patterned SOI wafers as an additional back-end-of-line process step [57-59]. Silicon nitride $\left(\mathrm{Si}_{3} \mathrm{~N}_{4}\right)$ is a mid-index contrast optics (MiDex) material, transparent from the visible $(\sim 0.4 \mu \mathrm{m})$ to the mid-IR $(4 \mu \mathrm{m})$ and has a lower thermo-optic coefficient compared to silicon. These material properties lead to waveguides with lower propagation loss, phase noise, backscattering, and sensitivity to temperature variation, which can be used for high quality and stable passive devices, such as filters $[60,61]$ and optical frequency comb generators [62] among others.

The fabrication compatibility of SOI and SNOI with the CMOS process is a major advantage of these material platforms over other alternatives, such as InP. From an economic perspective, silicon photonic manufacturing offers a low-cost solution for high-volume production by reusing the legacy CMOS infrastructure, with few or even no (zero-change) additional process steps. As opposed to microelectronics [63], the performance of most of the photonic building blocks does not scale with the critical dimensions of the fabrication process. However, they can benefit from lower process variations. While most of the feature size requirements are met with the $130-\mathrm{nm}$ or $90-\mathrm{nm}$ CMOS nodes (using 248 and $193 \mathrm{~nm}$ wavelength lithography, respectively), the 65- and 40-nm CMOS nodes can achieve better accuracy and therefore better performances and yield. This is due to the fact that these nodes make uses of immersion lithography and 300-mm wafers. Immersion lithography can substantially enhance the photolithography resolution and thus reduce the waveguide linewidth variations and lower the waveguide propagation loss. Larger wafers (300 vs. $200 \mathrm{~mm}$ ) benefit from lower silicon thickness variations [47]. Designers can then select the technology node offering the best trade-off between cost and performance.

Another key benefit of CMOS fabrication is the prospect of cointegration of photonics and microelectronics, for which various approaches have been demonstrated. Stateof-the-art monolithic cofabrication down to the $28 \mathrm{~nm}$ node process has been demonstrated [64], but this approach requires expensive and major process changes. Other approaches aim to decouple the photonic fabrication process from electronics so that each of them can be optimized [21]. The latter benefits from advanced nodes for high speed and efficient transistors, while the former can be fabricated at lower costs and higher yield on more mature CMOS lines. This approach of interfacing two wafers with electrical connections for heterogeneous photonic-microelectronic integration should remain the dominant paradigm in the immediate future. Various solutions for dual and multichip interfacing include wire bonding, flip-chip integration [6567], through silicon vias [68], or fine pitch copper pillars interconnect [69].

\subsection{Ecosystem and trends}

The silicon photonics market is growing at a fast pace and expected to accelerate in the near future [70]. While integrated device manufacturers, such as Intel, have their inhouse manufacturing capabilities to fabricate their selfdesigned PICs, a large number of fabless actors rely on a few open-access foundries with standardized processes and platforms. Therefore, the manufacturing players are evolving to the open-access model and translating the fabrication technology from research institutes to pureplay industrial foundries for high-volume throughput. In this model, pure-play foundries specialize only in the manufacturing steps. A detailed review of the open-access status is given in the study by Rahim et al. [22].

Different types of manufacturing process are offered to meet the needs of fabless designers, based on different material platforms, technology nodes, volumes of chips, turnaround time, and cost. For proof-of-concepts, rapid prototyping services (ex: AMO, Applied Nano Tool, Cornerstone, CNM/VLC, LIGENTEC) offer fast turnarounds but low functionalities and low volumes of chips, in part because their fabrication process often relies on e-beam lithography [71] instead of photolithography (exceptions include Cornerstone that uses $248 \mathrm{~nm}$ photolithography). 
CMOS pilot lines and research institutes propose lowvolume open-access manufacturing while improving the maturity level of their platform and process (ex: AIM, CEA-LETI, IMEC, IHP, LIGENTEC, Sandia Lab, VTT). Since 2018, pure-play industrial foundries (ex: AMF, CompoundTek, Global Foundry, LioniX, SilTerra, ST Micro, TSMC, TowerJazz) have granted open access to silicon photonic fabrication. They propose prototyping and R\&D through multiproject wafer runs, as well as low- to highvolume manufacturing for commercialization ramp up.

Silicon photonic manufacturing is advancing toward both standardization and diversification in terms of materials, layer thickness, and process. Standardization is essential for improved yield, increased system complexity, and reduced costs for the most common applications, such as telecommunications and data center interconnects. On the other hand, the diversification will be pushed by the emergence of other application markets, such as mid-IR sensing for chemical, biological, and trace gas monitoring, bioimaging in the visible, quantum photonics, highperformance computing, etc.

\section{Silicon coherent optical transceivers}

The quick evolution of the optical transport systems toward the elastic network paradigm has motivated the development of higher baud rate transmitters that can be dynamically adapted to various modulation formats and transmission distance. InP PICs and integrated coherent transceivers have achieved remarkable performance and commercial success in coherent optical transmission systems [72-75]. After decades of falling behind, silicon photonics has been able to catch up quickly over the last decade, thanks to the widely accessible foundry processes leveraging legacy CMOS facilities, as discussed in Section 2. Silicon photonics is well suited for monolithic coherent transceivers with performance comparable to discrete devices. Coherent transceivers require a variety of optical passive and active functions, all of which, except for the laser, can be tightly integrated on silicon chips. In this section, we first review the state-of-the-art silicon photonic coherent transmitters and receivers and discuss about the paths to higher speed operation through either higher baud rates or spectral/spatial superchannels. For system capacity scaling, important considerations, such as compatibility with ultrabroadband operation and scaling the bandwidth density, are presented near the end of this section.

\subsection{Single-carrier coherent transceivers}

\subsubsection{Monolithic coherent transceivers: state of the art}

Silicon photonic coherent transceivers are among the most successful commercial silicon photonic products. Figure 6 shows the typical configuration of a dual-polarization (DP) coherent optical transceiver fully integrated on a silicon chip. A continuous wave (CW) input is split into two paths, performing as a light source and local oscillator (LO) for the transmitter and receiver, respectively. The transmitter compromises two parallel IQ modulators for dual polarizations. The outputs of modulators are multiplexed using a polarization splitter-rotator (PSR) and coupled to the fibers through an edge coupler. Alternatively, a 2D grating coupler can replace the PSR and edge coupler for polarization multiplexing [76]. The coherent receiver consists of a PSR, two $90^{\circ}$ hybrid mixers, and four sets of balanced photodiodes (BPDs). The incoming complex optical signal interferes with the LO in the optical hybrid so that the inphase (I) and quadrature (Q) components can be separately measured using BPDs. Modern coherent systems use intradyne receivers [77], where the frequency difference between the transmitter and LO is within the signal bandwidth and can be estimated in the digital domain using CMOS application-specific integrated circuits.

Table 1 provides a summary of recently demonstrated monolithically integrated silicon photonic coherent transceivers. Acacia is a pioneer in commercializing silicon photonic coherent transceivers and recently reported their first $400 \mathrm{~Gb} / \mathrm{s}$ transmission over $6600 \mathrm{~km}$ in a field demonstration via a transatlantic submarine cable [78]. Elenion (recently acquired by Nokia) is another active player in this field. In 2018, they reported a high baud rate transceiver with a hybrid integrated tunable laser using an InP gain chip coupled to an external cavity on silicon (Figure 7(a)) [79]. At OFC 2020, NTT demonstrated a coherent optical subassembly (Figure 7(b)) that features a high bandwidth of over $50 \mathrm{GHz}$ in both the transmitter and receiver and has achieved up to 96 Gbaud in 16 QAM [80]. This high bandwidth has been enabled by a codesigned driver with variable gain and peaking control.

\subsubsection{Ge-on-Si photodetectors and coherent receivers}

Ge-on-Si photodetectors (PDs) have shown excellent performance in the telecommunication bands with high speed (>60 GHz), high responsivity (>1 A/W with over a 90\% quantum efficiency), and low dark current (down to the nA 


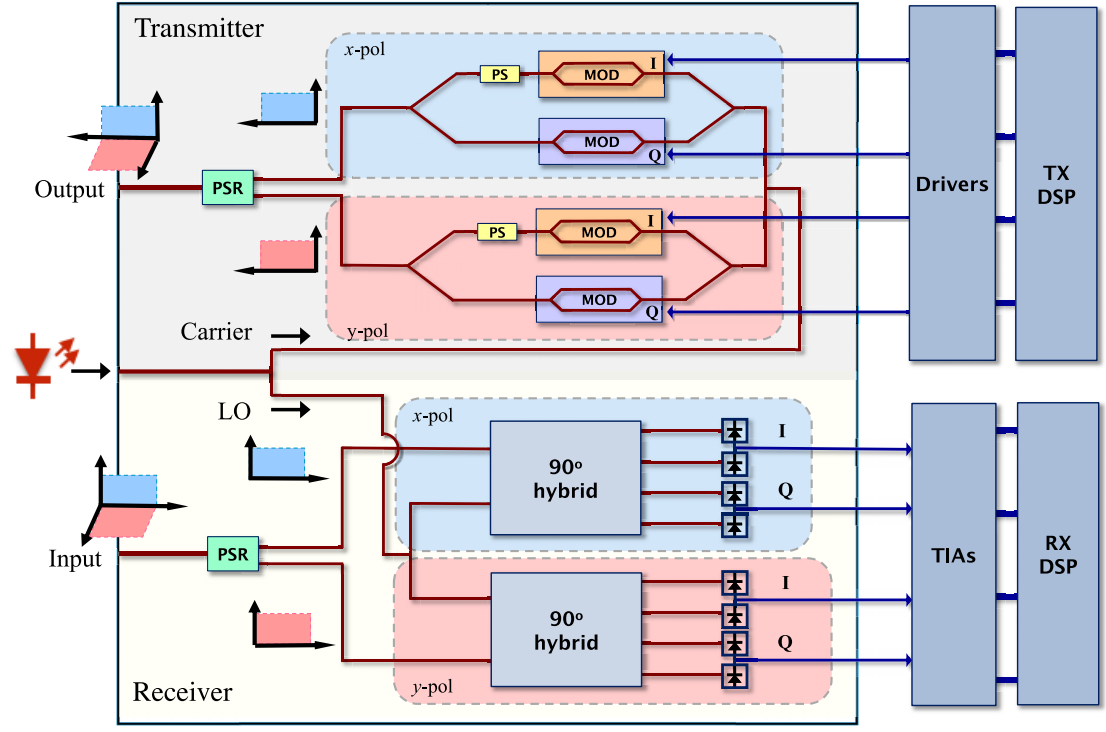

Figure 6: Block diagram of a fully integrated photonic coherent transceiver.

Table 1: Recent demonstrations of integrated silicon coherent optical transceivers.

\begin{tabular}{llll}
\hline Ref. & Year & Performance & Feature \\
\hline$[81,82]$ & 2016 & DP QPSK/16/64-QAM, 30 Gbaud & altrabroad band $(O, E, S, C, L)$ \\
{$[78]$} & 2020 & $400 \mathrm{~Gb} / \mathrm{s}$, near 70 Gbaud & $6.41 \mathrm{~b} / \mathrm{s} / \mathrm{Hz}$ over $6600 \mathrm{~km}$ \\
{$[79]$} & 2018 & DP QPSK/16-QAM, 64 Gbaud & Hybrid integrated tunable laser \\
{$[80]$} & 2020 & DP 16-QAM, 96 Gbaud & $50+$ GHz electro-opticelectro bandwidths
\end{tabular}

${ }^{a}$ The authors claimed 60 Gbaud under deployment with constellations up to 64 QAM for $600 \mathrm{~Gb} / \mathrm{s}$ net per wavelength [82]. QPSK, quadrature phase-shift keying.
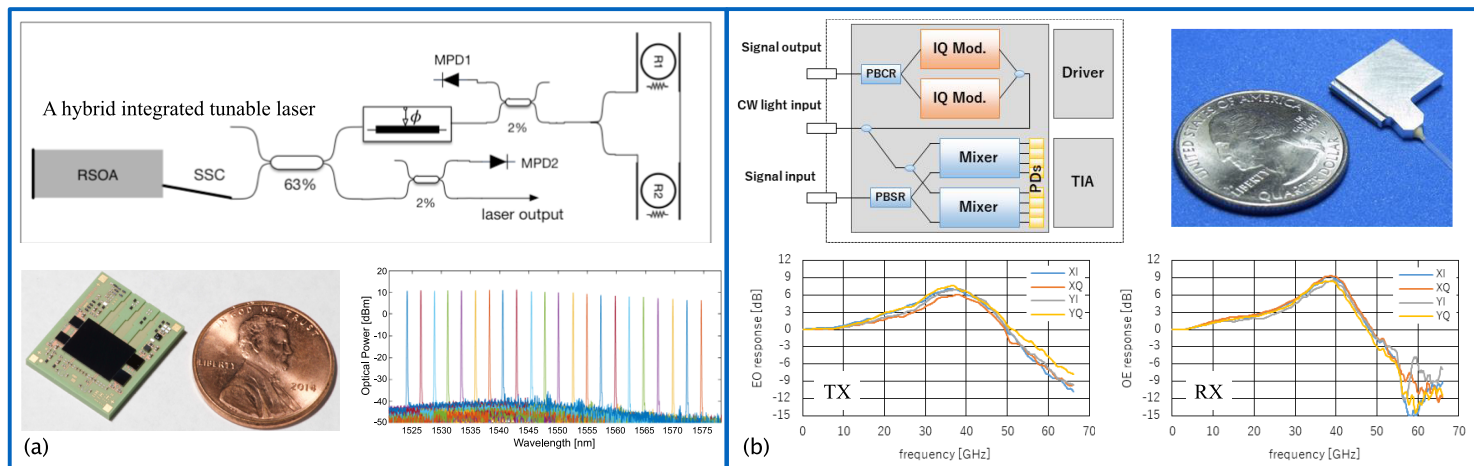

Figure 7: Silicon photonic coherent transceiver subassemblies. (a) a 64-Gbaud coherent transceiver with a hybrid integrated tunable laser [79] (top: block diagram of the hybrid integrated tunable laser; bottom left: photograph of the transceiver subassembly; bottom right: tuning performance of the laser); (b) a 95-Gbaud coherent transceiver with a bandwidth $>50 \mathrm{GHz}$ [80] (top left: block diagram of the coherent optical transceiver; top right: photograph of the packaged transceiver; bottom: transceiver (TX) and receiver (RX) frequency response).

level) [83-87]. Like other high-speed PDs, they are designed in a PIN diode structure operated at a zero or reverse bias, where the bandwidth of a PD is limited by (1) the carrier transit time through the depletion region and (2) the RC constant involving the junction and the load. The transit time-limited bandwidth $f_{t}$ is given as follows [88]

$$
f_{t r}=\frac{0.44}{\tau_{t r}}=0.44 \frac{v_{d}}{W}
$$


where $\tau_{t r}$ is the transit time dominated by the carrier drift in presence of an electrical field, $v_{d}$ is the drift velocity, and $W$ is the width of the depletion region. For example, $f_{t r}$ is greater than $65 \mathrm{GHz}$ for a 400-nm depletion region with a saturation drift velocity of $6 \times 10^{6} \mathrm{~cm} / \mathrm{s}$.

Both vertical and lateral junctions have been studied for high-frequency operations with respective advantages and drawbacks. Figure 8 shows one example for each case. The vertical junction typically has one contact formed on top of $n$-doped $\mathrm{Ge}$ and another on $p$-doped silicon. However, heavily doped Ge and metal-Ge contact induce optical absorption, resulting in a loss of responsivity. In addition, the metal-Ge contact also suffers from a higher resistance that limits the bandwidth [89], which can be improved by using a SiGe cover layer [90]. To address these issues, lateral junction structures without heavily doped Ge were developed, firstly on a largesection waveguide platform [86] and later on the submicron SOI [85, 91]. Eliminating the need for a Ge-doping process, the lateral junction design also simplifies the overall fabrication process. A high bandwidth of up to $67 \mathrm{GHz}$ for $100 \mathrm{~Gb} / \mathrm{s}$ in a silicon-contact lateral junction was reported [87]. Nevertheless, the cross section of the lateral junction design is usually very small (a few hundred $\mathrm{nm}$ to a couple of $\mu \mathrm{m}$ in width) to reduce $\tau_{t r}$, which limits its power handling capacity. In this sense, vertical junction is advantageous because $\tau_{t r}$ is only limited by the thickness of $\mathrm{Ge}$ and, therefore, the width can be as large as several $\mu \mathrm{m}$. This is particularly useful for coherent detection with a high input from the LO. In the study by Uetake et al. [90], a $8-\mu \mathrm{m}$-wide vertical junction
Ge-on-Si PD achieved a 40-GHz bandwidth for an input optical power of up to $5.8 \mathrm{dBm}$ while maintaining a high responsivity of near $1 \mathrm{~A} / \mathrm{W}$ and a low dark current density of $0.05 \mathrm{nA} / \mu \mathrm{m}^{2}$.

Ge-on-Si avalanche photodiodes (APDs) also attracted considerable attention for high-sensitivity receivers [9297]. Compared to PIN diodes, APDs usually have lower bandwidths and require more complex driving circuits, making them less popular for high-speed coherent detection. Nevertheless, they may be useful for circumstances when the link power budget is tight or a high-power LO is unavailable. Important figures of merit of APDs include gain-bandwidth product, bandwidth, and excess noise factor (depending on $k$, the ratio of the ionization coefficients of electrons and holes). To suppress the excess noise, an important consideration is to use silicon as the multiplication region for its exceptional $k$ value $(<0.1)$, while maintaining a low electrical field in the absorption region (Ge). The bandwidth is determined by the transit times over the absorption and avalanche multiplication regions and the avalanche built-up time. Separate absorption, charge, and multiplication (SACM) structures have been explored in both vertical $[92,93]$ and lateral junctions [94, 96]. A vertical junction SACM-APD achieved a gain-bandwidth product of $276 \mathrm{GHz}$ with a bandwidth of $23 \mathrm{GHz}$ [93]; the device shows more than $3 \mathrm{~dB}$ improvement in sensitivity at $100 \mathrm{~Gb} / \mathrm{s}$ PAM-4 [97]. An ultrahigh gainbandwidth product exceeding $430 \mathrm{GHz}$ was achieved using a lateral junction SACM structure; however, its bandwidth is less than $10 \mathrm{GHz}$, limited by the carrier drift time in the multiplication region [94]. A PIN-based APD was recently
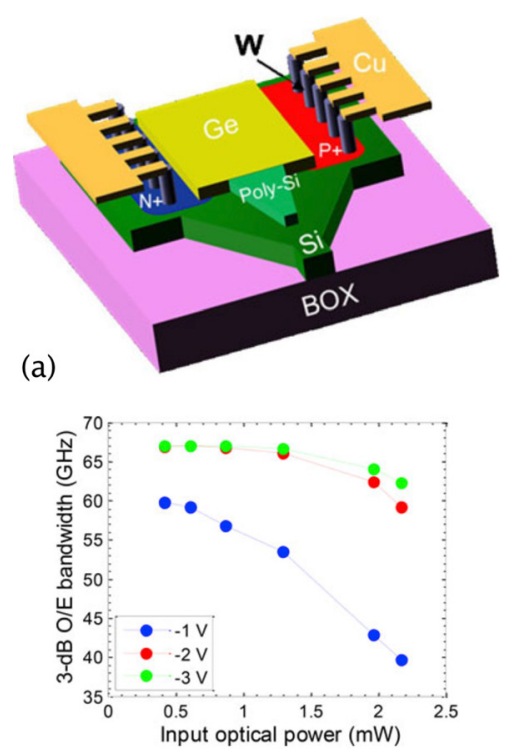

(b)

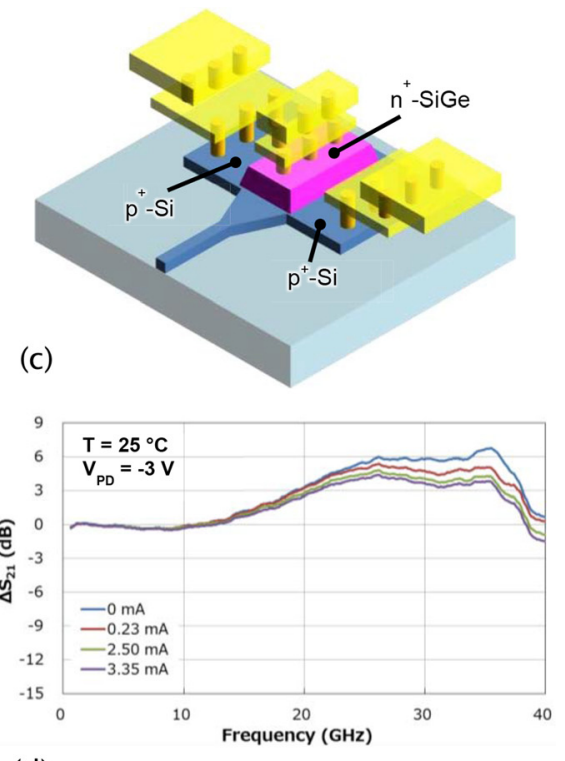

(d)
Figure 8: (a) Schematic of a lateral junction Ge-on-Si PD with (b) its 3-dB bandwidth as function of input power at various bias voltages [87]. (c) Schematic of a vertical junction Ge-on-Si PD with (c) its frequency response (measured with a transimpedance amplifier) at various input output powers [90]. 
demonstrated and achieved a bandwidth of up to $33 \mathrm{GHz}$ and 40-Gbaud, 16-QAM operation with a moderate effective $k$ of around 0.2 [96].

In addition to high-speed PDs, coherent receivers also require high-performance optical hybrids. Optical hybrids can be realized using a $4 \times 4$ multi-mode interferometer (MMI) $[98,99]$ or a $2 \times 4$ MMI serially connected with a $2 \times 2$ MMI [100, 101], as shown in Figure 9(a) and (b), respectively. They are fully passive and inherently suitable for optical hybrids because of the specific phase relation at outputs. Nevertheless, they are vulnerable to the wavelength dependence and fabrication errors due to the fact that the length of MMI couplers used in these hybrids is usually as long as hundreds of micrometers. An alternative way is to use a structure consisting of several compact components such as $1 \times 2 \mathrm{MMI}, 2 \times 2 \mathrm{MMI}$, and phase shifters (PSs). One example is shown in Figure 9(c) [102], where a $1 \times 2 \mathrm{MMI}$ separates the received signal to the I and $\mathrm{Q}$ arms of the receiver, meanwhile a 3-dB directional coupler (DC) splits the LO signal into these paths. In the I/Q arm, thermally tunable PSs enable precise control of the $90^{\circ}$ differential phase shift between the received signal and LO that are mixed by a 3-dB DC and then detected by a BPD. The study by Guan et al. [103] provides another example that has a similar configuration but with a more compact layout and optimized components (such as broadband $2 \times 2$ MMIs and $Y$ junction), avoiding the need for thermal PSs, as shown in Figure 9(d). The device has a compact footprint of $21.6 \times 27.9 \mu^{2}$ and an insertion loss of less than $0.5 \mathrm{~dB}$. The measured phase errors and common mode rejection ratio (CMRR) are of $<3^{\circ}$ and of $>30 \mathrm{~dB}$, respectively. Later on, a similar structure was also demonstrated using adiabatic $3 \mathrm{~dB}$ splitters/combiners instead of MMIs and achieved a phase error of $\angle 5^{\circ}$ and a CMRR of $>20 \mathrm{~dB}$ within the wavelength range of 1520-1620 $\mathrm{nm}$ [104].

Dual-polarization detection is routinely integrated with coherent receivers. It is implemented using monolithically integrated polarization splitters and rotators that will be reviewed in Section 4.1.

\subsubsection{Silicon IQ modulators}

Relying on the free-carrier plasma dispersion effect [106], silicon modulators suffer from relatively low efficiency and high optical attenuation. Therefore, there exists a stringent trade-off between the modulation efficiency and bandwidth. Challenges, design, and optimization of silicon modulators for general applications and, in particular, for pulse amplitude modulation form a broad and rich topic and have been detailed in recent review papers $[107,108]$. New materials heterogeneously integrated on silicon may effectively improve the modulation efficiency. However, when it comes to applications that require complex integrated circuits such as monolithically integrated coherent transceivers, other factors such as compatibility with a large variety of passive and active components and the overall yield and cost often become more crucial. Therefore, all-silicon modulators are preferred for a majority of applications that require compact, cost-efficient optical transceivers.

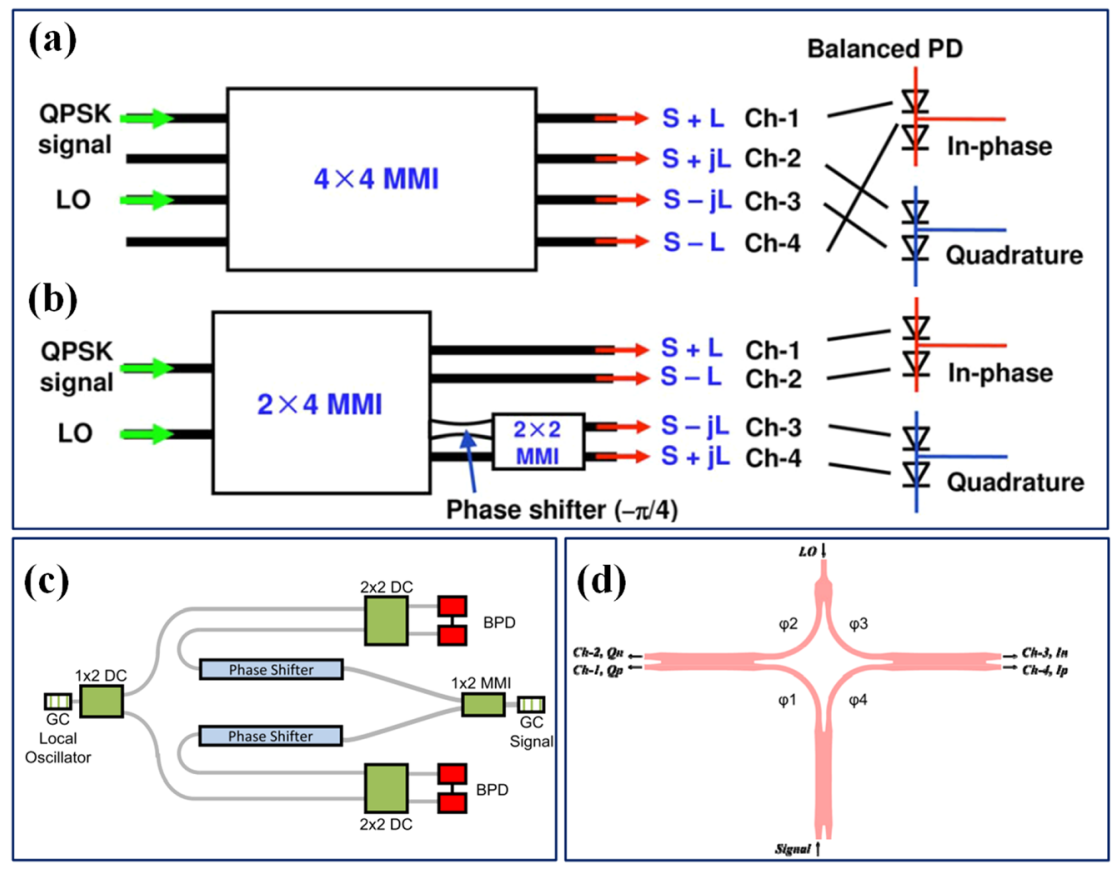

Figure 9: Schematic diagram of (a) optical hybrid based on a $4 \times 4 \mathrm{MMI}$ and (b) optical hybrid based on a $2 \times 4 \mathrm{MMI}$ serially connected with a $2 \times 2$ MMI [105]; (c) optical hybrid based on $1 \times 2 \mathrm{MMI}, 2 \times 2 \mathrm{MMI}$, and phase shifters [102]; (d) optical hybrid comprising one $Y$-junction and three $2 \times 2$ MMIs [103]. 
The system-level performance of a silicon modulator in a transmission link can be characterized by the modulator power penalty (MPP) [107] with contributions from (1) optical attenuation due to the waveguide background loss (dominated by sidewall scattering), free-carrier absorption (caused by waveguide doping for optical modulation) [106], and nonlinear optical processes such as two-photon absorption [109], (2) modulation loss due to a voltage swing smaller than $V_{\pi}$, and (3) intersymbol interference due to limited electro-optic bandwidth. These three penalty sources are intricately intertwined. Therefore, an optimal design for a given process can only be achieved by minimizing the total power penalty. A bandwidth-aware figure of merit (FOM) was recently proposed for silicon modulators in a pulse-amplitude modulation (PAM) link [107, 110] to minimize this penalty. A numerical approach was used for modulator design optimization (minimum MPP) for coherent transmissions at $400 \mathrm{~Gb} / \mathrm{s}$ and beyond [111].
Recent efforts have led to record-breaking demonstrations of coherent optical transmissions using all-silicon modulators for line rates of up to $1 \mathrm{~Tb} / \mathrm{s}$ in dual-polarization higher-order QAM with a spectral efficiency of $8.4 \mathrm{bits} / \mathrm{s} / \mathrm{Hz}$ (4.2 bits/s/Hz per polarization) [112]. Long-distance transmissions of 400-600 Gb/s net were also achieved for over $1500 \mathrm{~km}$ (Figure 10) [35]. We have seen a clear trend toward even higher symbol rates. Recent works have achieved up to 120 Gbaud 16-QAM [113] and 100 Gbaud 32-QAM transmissions [114]. Some recent results of coherent optical transmissions using silicon photonic transmitters are summarized in Table 2. For comparison, some recently demonstrated hybrid silicon modulators are also included in the table.

Silicon modulators also suffer from nonlinear patterndependent behavior that causes signal distortion in the presence of very high-order modulations or large driving voltages. It is higher than conventional $\mathrm{LiNbO}_{3}$ Mach-

Table 2: Some state-of-the-art demonstrations of high baud rate integrated silicon IQ modulators.

\begin{tabular}{|c|c|c|c|c|c|c|c|}
\hline Ref. & Year & Process & Format & Symbol rate (Gbaud) & Line rate (FEC overhead) & Link & Net SE/Pol ${ }^{\mathrm{a}}$ (bits/s/Hz) \\
\hline \multirow[t]{2}{*}{ [112] } & 2020 & Lateral $p-n$ & SP 32-QAM & 100 & $1 \mathrm{~Tb} / \mathrm{s}(20 \%)$ & $\mathrm{B} 2 \mathrm{~B}$ & 4.2 \\
\hline & & & SP 16-QAM & 100 & $800 \mathrm{~Gb} / \mathrm{s}(7 \%)$ & $\mathrm{B} 2 \mathrm{~B}$ & 3.8 \\
\hline \multirow[t]{2}{*}{ [113] } & 2020 & Lateral $p-n$ & SP 32-QAM & 100 & $500 \mathrm{~Gb} / \mathrm{s}(24 \%)$ & $\mathrm{B} 2 \mathrm{~B}$ & 4.0 \\
\hline & & & SP 16-QAM & 120 & $480 \mathrm{~Gb} / \mathrm{s}(15 \%)$ & $\mathrm{B} 2 \mathrm{~B}$ & 3.5 \\
\hline [115] & 2019 & Lateral $p-n$ & SP 256-QAM & 20 & $120 \mathrm{~Gb} / \mathrm{s}(20 \%)$ & $\mathrm{B} 2 \mathrm{~B}$ & 6.7 \\
\hline \multirow[t]{2}{*}{ [35] } & 2018 & Lateral $p-n$ & DP 32-QAM & 72 & $720 \mathrm{~Gb} / \mathrm{s}(20 \%)$ & $480 \mathrm{~km}$ & 4.2 \\
\hline & & & DP 16-QAM & 60 & $480 \mathrm{~Gb} / \mathrm{s}(20 \%)$ & $1520 \mathrm{~km}$ & 3.3 \\
\hline \multirow[t]{2}{*}{ [116] } & 2017 & SISCAP & DP 16-QAM & 40.13 & $321.4 \mathrm{~Gb} / \mathrm{s}(20 \%)$ & $\mathrm{B} 2 \mathrm{~B}$ & 3.3 \\
\hline & & & & 32.17 & $257.3 \mathrm{~Gb} / \mathrm{s}(20 \%)$ & $1214 \mathrm{~km}$ & 3.3 \\
\hline [117] & 2018 & $\mathrm{SOH}^{\mathrm{c}}$ & SP 16-QAM & 100 & $400 \mathrm{~Gb} / \mathrm{s}(20 \%)$ & $\mathrm{B} 2 \mathrm{~B}$ & 3.3 \\
\hline [118] & 2019 & $\mathrm{POH}^{\mathrm{d}}$ & SP 16-QAM & 100 & $400 \mathrm{~Gb} / \mathrm{s}(20 \%)$ & B2B & 3.3 \\
\hline
\end{tabular}

${ }^{a}$ Nyquist bandwidth is used in the calculation of spectral efficiency (SE) per polarization (Pol) for simplicity, although the actual pulse shaping filters used in experiments may be different.

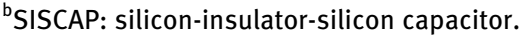

'SOH: silicon-organic hybrid.

${ }^{\mathrm{d}} \mathrm{POH}$ : Plasmonic-organic hybrid.

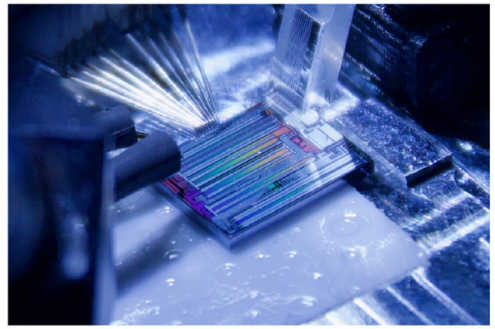

(a)

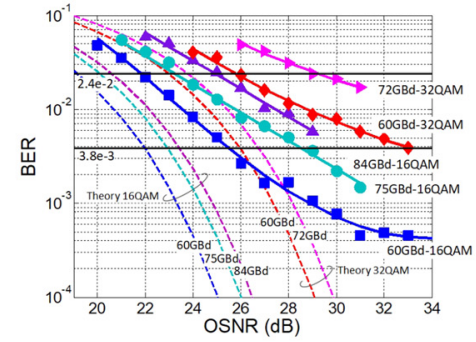

(b)

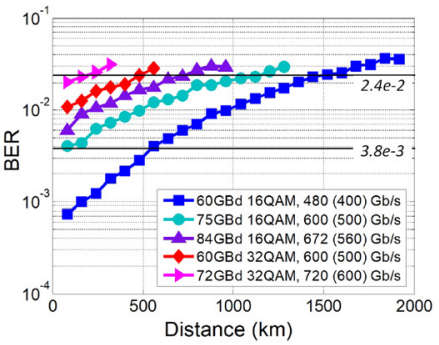

(c)

Figure 10: (a) Photograph of a silicon IQ modulator under test. (b) Back-to-back transmission performance for 60/75/84 Gbaud 16QAM and 60/72 Gbaud 32QAM. Dashed lines: theoretical prediction for an ideal transceiver system. (c) Transmission performance over distance for 60/ 75/84 Gbaud 16QAM and 60/72 Gbaud 32QAM. Raw bit rates are reported in the legend and post-FEC overhead bit rates in parentheses [35]. 
Zehnder modulator (MZM) due to the nonlinear dynamic response of the silicon PS in which the modulation bandwidth increases with reverse voltage across the depletion region $[119,120]$. To address this issue, a digital predistortion method has been developed based on the iterative learning control technique [121] and a behavior model [115]. Applying these methods with the silicon IQ modulator, very-high-order QAM operations, such as 256-QAM at 20-Gbaud and 128-QAM at 40-Gbaud, have been achieved with a spectral efficiency of up to $6.7 \mathrm{bit} / \mathrm{s} / \mathrm{Hz}$ in single polarization [115, 121].

\subsubsection{How far can we push the speed of a silicon transceiver?}

As next-generation commercial coherent transceivers will soon go beyond $100 \mathrm{Gbaud}$, it is pertinent to explore when we will hit the ceiling of speed in silicon photonics. Ge PDs for direct and coherent detection are among the most mature silicon photonic devices. As already discussed in Section 3.1.2, recent progress in Ge-on-SOI waveguide photodetectors has achieved a bandwidth of greater than $60 \mathrm{GHz}$ with a responsivity close to $1 \mathrm{~A} / \mathrm{W}[87,90,122,123]$. A bandwidth beyond $70 \mathrm{GHz}$ and even approaching $100 \mathrm{GHz}$ should be feasible with a moderate compromise of responsivity and power consumption. Constraints shall come more from the transmitter side.

The speed of a depletion-mode silicon photonic modulator depends on the $p n$-junction dynamics (carrier dynamics and RC constant), radio frequency (RF) loss (e.g., in traveling-wave [TW] electrodes), photon lifetime (in optical resonator and slow light waveguides), and mismatch between RF and optical waves. The intrinsic bandwidth of a reverse-biased $p n$-junction embedded in a silicon waveguide, depending on the junction's design and bias voltage, can readily go beyond $100 \mathrm{GHz}$ and typically does not limit the overall speed of the modulator. The other limiting factors put more constraints on the optical modulation speed. Photon lifetime is usually the primary limiting factor in resonator modulators. For example, a recently demonstrated resonator modulator using a lumped electrode shows an electrical bandwidth near $150 \mathrm{GHz}$ and a photon life time-limited bandwidth of about $50 \mathrm{GHz}$ [124].

Most demonstrated all-silicon IQ modulators apply TW electrodes to drive long PSs in MZM configuration to lower the driving voltage, where the bandwidth is limited by the loss (on the order of $1-10 \mathrm{~dB} / \mathrm{mm}$ ) of driving signals along the microwave transmission lines loaded by $p n$-junctions [111]. Bandwidths ranging from 30 to $60 \mathrm{GHz}[107,111,125$, 126] have been reported for all-silicon TW-MZMs, depending on the bias and the length of the modulator. Novel driving schemes and modulator designs are necessary to bypass the fundamental trade-off between modulation efficiency and bandwidth, requiring a higher complexity in electronic-photonic integrated circuits.

Segmenting the driving electrode provides a convenient solution for this trade-off. In the conventional TW modulator, the length of the PS limits the bandwidth, due to RF losses alongside the TW electrode and the velocity mismatch between the electrical and optical signals [110]. However, in the segmented modulator, the driving signals are applied sequentially to the segments with precisely tunable timing circuits to match the optical signal delay between different segments. As a result, the interaction length can be increased and the driving voltage can be lowered. Weighted binary codes can be used to determine the length of each segment for a minimized number of segments, simplifying the driving configuration. This binary-weighted segmentation (BWS) scheme has been examined for short-reach links with direct detection [107, 125, 127], where a PAM signal can be achieved using binary inputs directly applied to the RF drivers without a digital-to-analog converter (DAC). This scheme eliminates the need for an electrical DAC but makes it impossible to apply advanced DSP such as preemphasis and pulse shaping that have been crucial enablers of highcapacity coherent transmissions. To generate higher-order modulation formats (such as QAM), a PAM signal generated by a DAC can be applied to the MZM segments, which allows for the use of advanced DSP. Despite the addition of a DAC, the BWS segmentation allows the usage of low-cost, lowresolution DACs to achieve higher signal quality by generating higher modulation formats using lower order PAM signals. For example, to generate QAM-64, we can use either two segments with PAM-4 or one segment with PAM-8 (the conventional design).

Preemphasis is also an efficient means to compensate the limited bandwidth of the DAC and the silicon modulator. The use of an optical filter can reduce the burden of the digital preemphasis and lower quantization noise. Independently designed digital and optical filters cannot achieve the best trade-off of DAC quantization noise and SNR degradation across the signal frequency band. A joint optimization procedure of optical and digital preemphasis filters has proven to be able to effectively improve the signal quality [128]. Although the optical emphasis was implemented using a bench-top programmable optical filter, it can also be integrated on the silicon chip. A monolithically integrated optical preemphasis was demonstrated recently using a cascaded Mach-Zehnder interferometer (MZI) structure [129].

Realizing a transceiver system involves complex tradeoff between performance, energy consumption, and 
economy. Considering that heterogeneous integration of exotic materials onto silicon still faces the challenges of yield and cost, it is expected that researchers and the industry will continue to push the bandwidth of all-silicon modulators toward their physical limit. An analysis based on the carrier transit time across the depletion region in a typical depletion-mode silicon modulator predicted a bandwidth of $90 \mathrm{GHz}$ capable of achieving 140 Gbaud [130]. The overall bandwidth of a single-carrier silicon photonic transceiver will thus be limited by electronic functions such as a DAC and analog-to-digital converter or, in other words, the Moore's law or its end. At this point of time, it seems reasonable yet ambitious to envision a $1.6 \mathrm{~Tb} / \mathrm{s}$ silicon photonic transceiver in the coming years.

\subsection{Superchannel transceivers}

Beyond $40 \mathrm{GHz}$, the performance of the optical transmitter starts being severely limited by electronics such as the DAC and drivers. Signal multiplexing is needed to overcome this barrier. In this sense, the concept of superchannel [33] provides a promising solution for 100 Gbaud and beyond. A superchannel combines a number of subcarriers for a wide-band signal, each subcarrier being modulated at an electrically affordable baud rate. A configuration of a multisubcarrier superchannel transmitter is illustrated in Figure 11(a). For example, an aggregate $1.24 \mathrm{~Tb} / \mathrm{s}$ line rate (net rate of $1 \mathrm{~Tb} / \mathrm{s}$ ) can be achieved by multiplexing four optical carriers each carrying a subchannel of 40-Gbaud 16-QAM in two polarizations.
Different from the conventional WDM technique, a super-channel can be implemented with a zero guard band between adjacent optical carriers to maximize the spectral efficiency, e.g., using Nyquist-WDM for a seamless spectrum [32, 33]. Another remarkable advantage of the superchannel technique is its compatibility with the paradigm of elastic optical networking where the fixed frequency grid of conventional WDM systems is replaced by dynamic bandwidth allocation [131]. Compared to the single-carrier solution, this superchannel architecture also allows for a lower order modulation format (e.g., 16-QAM16 versus 64-QAM) for transmission over a longer distance. It also helps improve the tolerance to chromatic dispersion and nonlinear impairments [132, 133].

An MZM-based optical frequency comb generator was recently demonstrated on a silicon chip [134]. Using this integrated comb, an $800 \mathrm{~Gb} / \mathrm{s}$ superchannel (five carriers with 20-GBaud DP-QAM-16) was achieved, indicating a promising source solution for few-carrier superchannel transmitters. Monotonically integrated superchannels have been successfully demonstrated on $\operatorname{InP}$ for $C+L$ band transmission capable of supporting $1.2 \mathrm{~Tb} / \mathrm{s}$ per channel with six subcarriers [135] . To date, no fully integrated silicon superchannel transceiver has been demonstrated, largely due to the challenges of integration of lasers and amplifiers. With the rapid progress in integrated multiwavelength laser sources and the increasing demand for bandwidth, we expect to see a silicon photonic superchannel transceiver in the coming years.

A superchannel can also be implemented in the paradigm of SDM by grouping a number of spatial paths for

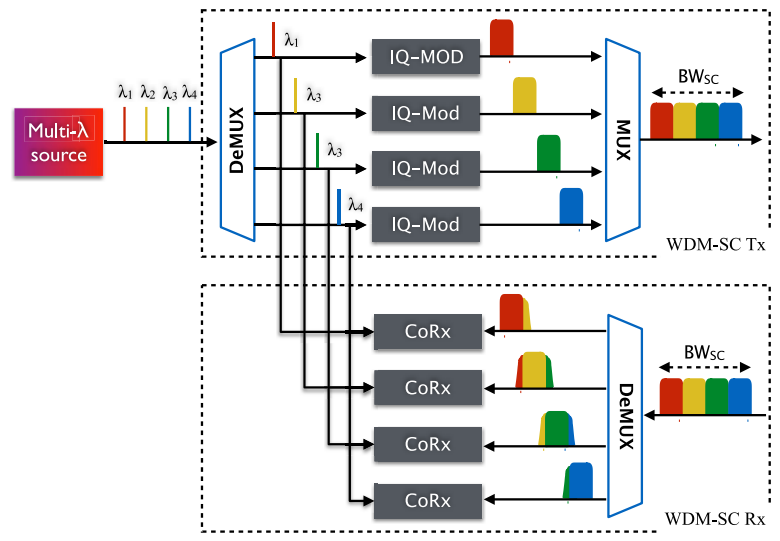

(a)

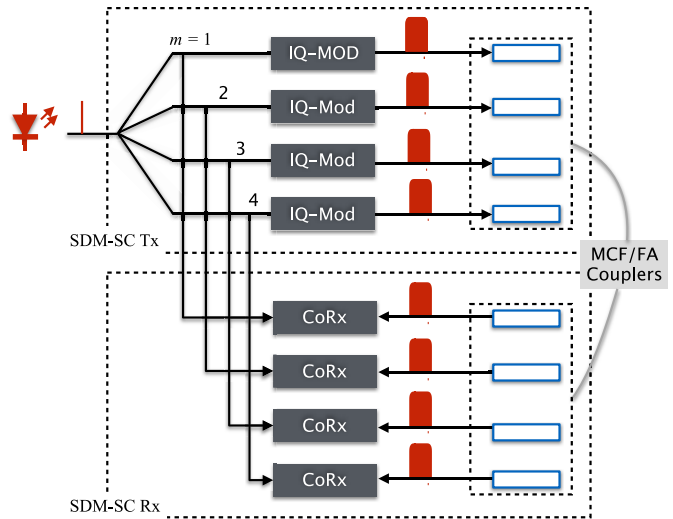

(b)

Figure 11: (a) Schematic of a spectral superchannel transceiver. Multiwavelength source can take the form of optical frequency comb or laser bank. The deMUX at the input side is not needed in the later case. (b) Schematic of a spatial superchannel transceiver for a single wavelength carrier. Only one polarization path is shown for simplicity. 
dynamic bandwidth allocation in a flexible optical network [136]. In a simple case as illustrated in Figure 11(b), a single laser output is split into a number of spatial subcarriers that can either be individual fibers coupled to the chip through a fiber array or different cores of a multicore fiber (MCF). Without using wavelength (de-)MUX, the architecture of the spatial superchannel (Figure 11(b)) appears to be simpler than that of spectral superchannel (Figure 11(a)). However, for a WDM $\times$ SDM system (Figure 2), the overall transceiver system complexity should be the same or similar for a given spectral efficiency.

Despite the advantages, the superchannel imposes a higher complexity in photonic circuits and RF connections. High-performance integrated wavelength- and spacedivision multiplexers are needed for superchannel transceivers, which will be discussed in Section 4. In addition, $\mathrm{RF}$ and optical crosstalks between subchannels may cause signal distortion leading to power penalty in DSP compensation.

\subsection{Compatibility with ultrawide-band systems}

Silicon photonic transceivers can well support the longterm evolution of ultrawide-band transmission systems. Crystalline silicon is transparent in a very wide spectral range from 1.0 to $8.5 \mu \mathrm{m}$ [137], well covering all the transmission bands of conventional single-mode fiber ranging from 1260 to $1675 \mathrm{~nm}$ (i.e., $54 \mathrm{THz}$ including $O, E, S, C, L$ and $U$ bands). Recent breakthroughs in low-loss photonic crystal hollow-core fibers have raised great interest in a transmission window around $2 \mu \mathrm{m}$, falling within the amplification range of thulium-doped fiber amplifiers $(1700-2100 \mathrm{~nm})[138,139]$, which promises a significant increase in the spectral resource [140]. To date, only a very limited amount of transmission experiments at relatively low rates were demonstrated $[141,142]$ due to the immaturity of the fiber and the lack of high-performance electrooptic components in this new band.

Thanks to the broadband nature of the free-carrier plasma dispersion effect, silicon modulators can work in a very broad range [143]. In a hero demonstration, $240 \mathrm{~Gb} / \mathrm{s}$ 16-QAM was achieved with a commercial silicon photonic coherent transceiver over a 370-nm wavelength span from $O$ to $L$ bands [81], as shown in Figure 12. High-speed silicon modulators in the depletion mode at $1950 \mathrm{~nm}$ were recently demonstrated [144, 145]. Although the data rates achieved so far were limited by available detection systems, the performance of these devices is expected to be comparable to their counterparts in $O$ and $C$ bands.

Performance of a silicon transceiver at longer wavelengths is limited by the Ge photodetectors whose responsivity shows a sharp roll-off after $1600 \mathrm{~nm}$ due to the bandgap of Ge grown on silicon (around $0.782 \mathrm{eV}$ ) [84]. Heterogeneously integrated InGaAs-on-Si photodetectors have shown exceptional performance $[84,146]$ but require complex wafer bonding processes that are generally not available in CMOS-photonics foundries. Fortunately, a resonator structure can significantly enhance the responsivity [147]. Assisted by a whispering-gallery cavity, a Geon-Si photodetector has achieved a responsivity of up to $0.45 \mathrm{~A} / \mathrm{W}$ at $1630 \mathrm{~nm}$ [148]. Toward even longer wavelengths, GeSn alloys grown on silicon have attracted a lot of attention [149, 150]. Recent works toward mid-IR imaging have extended the cut-off wavelength to $3.65 \mu \mathrm{m}$ [149].

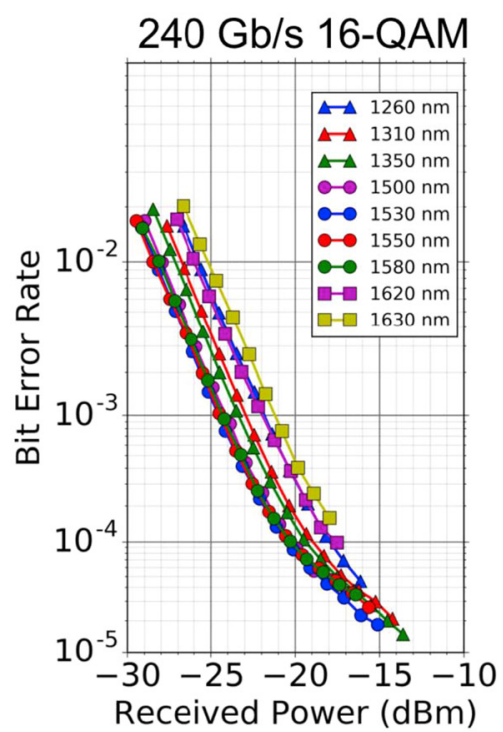

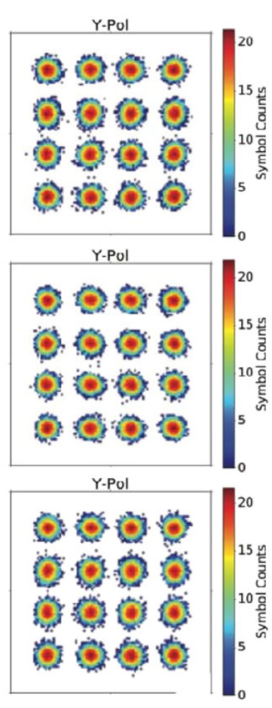

Figure 12: Performance of a commercial silicon photonic coherent transceiver over $370 \mathrm{~nm}$ at 240-Gb 16-QAM [82]. 
GHz-level GeSn-on-Si photodetectors were reported with a responsivity of $0.04 \mathrm{~A} / \mathrm{W}$ at $1900 \mathrm{~nm}$ and a dark current density of $31 \mathrm{~mA} / \mathrm{cm}^{2}$ [150]. Another interesting approach makes use of midgap states in silicon through implantation-induced defects, by which $20 \mathrm{~Gb} / \mathrm{s}$ at $1960 \mathrm{~nm}$ with a responsivity of $0.3 \mathrm{~A} / \mathrm{W}$ has been achieved [151]. Tensile strain in Ge can extend its wavelength responsivity; a recent work has achieved a responsivity of $0.31 \mathrm{~A} / \mathrm{W}$ at $1850 \mathrm{~nm}[152]$.

\subsection{Scaling the bandwidth density}

A high-capacity transmission system requires a large number of coherent transceivers (Figure 3). As the number of SDM-WDM channels increases, footprints of photonic chips become a crucial consideration. The essential question is how much bandwidth we can pack into a unit on-chip real estate (i.e., 2D bandwidth density in $\mathrm{Gb} / \mathrm{s} / \mathrm{mm}^{2}$ ). In extreme bandwidth-dense applications such as hyperscale data center switches $[153,154]$ where optical interconnect chips are copackaged with electronic switching circuits, the interface bandwidth density (i.e., 1D bandwidth density in $\mathrm{Gb} / \mathrm{s} / \mathrm{mm}$ ) becomes also crucial. Careful considerations are required to minimize $\mathrm{RF}$ crosstalk in densely integrated PICs. Ultracompact transceivers will be essential for the continuous scaling of bandwidth density.

\subsubsection{RF crosstalk}

Severe RF crosstalk may occur when two high-frequency electro-optic devices are placed closely on the same chip. In the study by Jiang et al. [155], a crosstalk of $-20 \mathrm{~dB}$ for frequencies greater than $10 \mathrm{GHz}$ was measured for a pair of parallel silicon TW-MZMs with a separation distance of $200 \mu \mathrm{m}$, resulting in a considerable OSNR penalty (about $2 \mathrm{~dB}$ ). A similar crosstalk was also reported in InP modulators [156]. It was shown that the impact on OSNR penalty becomes negligible when the separation is increased to $600 \mu \mathrm{m}$ with an RF cross talk of near $-30 \mathrm{~dB}$ [155].

The RF crosstalk also causes signal degradation in an IQ modulator, which put a constraint on its footprint. For example, in the study by Zhalehpour et al. [112], a spacing of $700 \mu \mathrm{m}$ between the I and Q branches is used in an IQ modulator, achieving a bandwidth density of $596 \mathrm{~Gb} / \mathrm{s} / \mathrm{mm}$ at a net data rate of $833 \mathrm{~Gb} / \mathrm{s}$. In the study by Chen et al. [157], DSP is applied to compensate for the strong coupling between I and Q branches of a silicon IQ modulator, leading to a $4.4 \mathrm{~dB}$ improvement in $Q^{2}$ factor on 64-QAM signals.

\subsubsection{Ultracompact, low-power devices}

While Ge-on-Si waveguide photodiodes are very compact (on the scale of $10 \mu \mathrm{m}$ in length), silicon optical modulators often require long PSs on the millimeter scale, not to mention the large spacing between driving electrodes due to RF crosstalk, as discussed above. Therefore, there exists a strong motivation to develop ultracompact silicon optical modulators. While long-reach transmissions systems have a less stringent power budget than short-reach applications such as intradata center optical interconnects, energy consumption or efficiency (in bits per joule) of optical transceivers is becoming increasingly important as the system scales up. Silicon modulators also dominate over detectors in energy consumption that should be lowered as their footprints. The energy efficiency of silicon TW-MZMs is inferior to that of the state-of-the-art $\mathrm{LiNbO}_{3}$ and InP modulators due to the relatively high $V_{\pi}$ and the inherent loss from the plasma dispersion effect. Their modulation efficiency is typically on the order of $100 \mathrm{fJ} / \mathrm{bit}$ to $1 \mathrm{pJ} / \mathrm{bit}$, depending on the modulation formats and driving configuration [127].

Resonator modulators provide an effective means of reducing the footprint and energy consumption, among which microring modulators (MRMs) [158-160] are most popular due to their easy implementation and low back reflections. Compared to the TW-MZMs, their energy efficiency can be improved by 10-100 times and down to the $\mathrm{fJ} / \mathrm{bit}$ level $[159,160]$. Bandwidth densities of over $1 \mathrm{~Tb} / \mathrm{s} /$ $\mathrm{mm}^{2}$ have been demonstrated using WDM MRM hybrids integrated with $14 \mathrm{~nm}$ FinFET [161]. In addition, MRMs can work simultaneously as MUX and modulators, thus greatly simplifying WDM transmitters $[162,163]$. However, resonator modulators suffer from impairments such as strong nonlinearity and chirp $[160,164]$. In the study by Dong et al. [164], a $56 \mathrm{~Gb} / \mathrm{s}$ QPSK generated by silicon microring modulators is demonstrated, where resonator-induced chirp has been identified as the factor limiting the overall performance. The chirp issue can be largely addressed using a dual-ring push-pull configuration [165] that has been proven effective in enabling a longer distance PAM transmission [166] but not yet demonstrated to date for coherent transmission.

There are in general two groups of nonlinear responses in a silicon modulator: (1) the static nonlinearity due to the spectral transmission shape determined by the optical transfer function of the interferometer (for MZM) or resonator (for MRM) and (2) dynamic nonlinearity due to the 
voltage-dependent frequency response that is stronger in MRMs compared to MZMs because the frequency response of an MRM is strongly dependent on the detuning from its resonance [160]. Both of them can be compensated digitally as shown in a recently demonstrated $112 \mathrm{~Gb} / \mathrm{s}$ PAM-4 MRM with a CMOS driver [167]. In addition, thermal stabilization (including the self-heating effect in presence of a high-power optical input $[168,169])$ and limited optical bandwidth are well-known challenges facing MRMs.

Slow light [170] provides another solution for compact, low-power modulators through enhanced light-matter interactions that can shrink the footprint of silicon modulators to the $100-\mu \mathrm{m}$ scale. Well-engineered slow-light waveguides allow for modulation enhancement, as well as operation insensitive to temperature fluctuations [171-173]. The enhancement in modulation efficiency is characterized by the enhancement factor $y$ defined as the ratio of group index of a slow-light waveguide over that of the conventional silicon rib waveguide (around 3.8), which can also be explained as the proportional decrease in the FOM $V_{\pi} \cdot L$. Using 2-D photonic crystal waveguides, a high group index in the range of 8-9 $(y>2)$ is reported over $42 \mathrm{~nm}$ covering the entire $C$-band; $25 \mathrm{~Gb} / \mathrm{s}$ was achieved with a shifter of $200 \mu \mathrm{m}$ in length [171]. An IQ modulator using 2D photonic crystal waveguides (Figure 13(a)) achieved 15 GBaud QPSK modulation [174]. Nevertheless, fabrication of 2-D photonic crystals is still challenging using optical lithography.

One-dimensional periodic photonic structures such as Bragg gratings are relatively easier for fabrication $[175,176]$. In the study by Brimont et al. [177], an MZM based on Bragg grating waveguides was demonstrated (Figure 13(b)). Working on the photonic band edge, the modulator is characterized by a group index of $8(\gamma \sim 2)$ over an optical bandwidth of $1.3 \mathrm{~nm}$. Cascaded resonators can also slow down light effectively, where low-quality factor cavities with a short photon lifetime can be designed for broader optical bandwidth [172, 173]. These resonators are usually overcoupled for simultaneous achievement of enhanced phase modulation and a broader optical bandwidth (and thus a broader temperature operational range). In the study by Romero-García et al. [172], an MZM with each branch coupled to a number of microring resonators (Figure 13(c)) achieved an enhancement $y \sim 7$ and an optical bandwidth of $3.8 \mathrm{~nm}$. Slow-light modulators in cascaded phase-shifted Bragg gratings were demonstrated $[173,178]$, which are essentially coupled resonators in 1D photonic crystals. In the study by Jafari et al. [173], an MZM assisted by cascaded phase-shifted Bragg gratings (Figure 13(d)) achieved an enhancement $\gamma \sim 7$ over an optical bandwidth of $3.5 \mathrm{~nm}$. Nevertheless, slow-light modulators usually suffer from a higher passive loss that scales nonlinearly with the group index $[179,180]$ and increased velocity mismatch between optical and RF signals, indicating a more complex trade-off between efficiency, loss, and bandwidth and requiring a thorough optimization in a system context.

Given the challenge of achieving high-bandwidth, low-power modulators in pure silicon, heterogeneous integration of advanced materials onto silicon for optical modulation has attracted considerable attention.

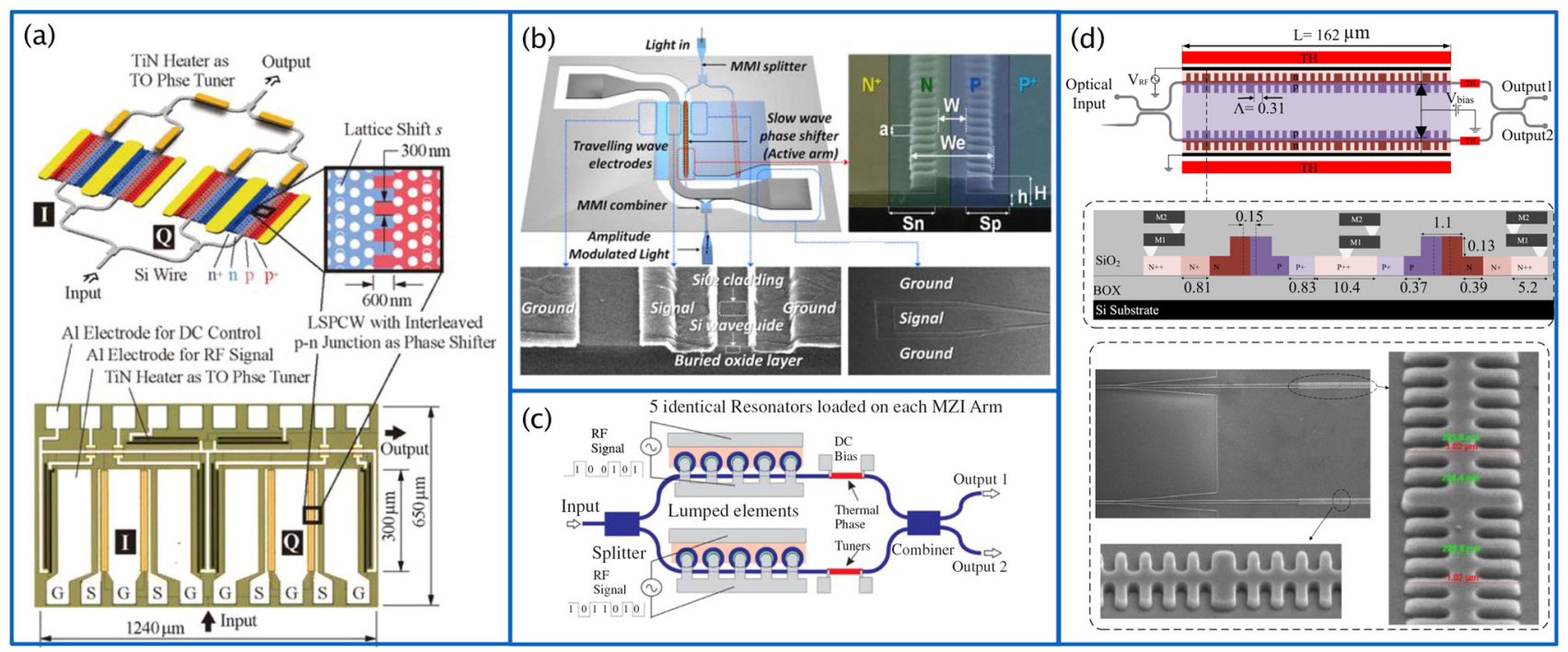

Figure 13: Slow light modulators.

(a) IQ modulator in 2D photonic crystals with interleaved $p$ - $n$ junctions [174]; (b) Bragg grating modulator working in the slow-light region (i.e., at the photonic band edge) [177]; (c) MZM modulator using slow-light waveguides in cascaded overcoupled microring resonators [172]; (d) MZM modulator using cascaded phased-shifted Bragg gratings (i.e., coupled 1D photonic crystals) [173]. MZM, Mach-Zehnder modulator. 
Exceptional performance has been demonstrated using InP $[181,182]$ and lithium niobate thin films [183] through wafer bonding and silicon-organic hybrid (SOH) [117] and plasmonic-organic hybrid ( $\mathrm{POH})$ structures [118]. Using SOH and POH IQ modulators, 100-Gbaud 16-QAM has been demonstrated [117, 118, 184]. An extremely compact $(4 \times 25 \times 3 \mu \mathrm{m})$ IQ modulator with an ultralow modulation energy consumption on the femtojoule-per-bit level has achieved $400 \mathrm{~Gb} / \mathrm{s}$ [118]. Other new materials, including 2D materials such as graphene [185], ferroelectric materials such as lead zirconate titanate [186] and barium titanate [187], and transparent conductive oxide such as ITO [188, 189], also hold great promises for compact, low-power optical modulators. Heterogeneous integration on silicon photonics involves rich physics and engineering techniques [190], which is beyond the scope of this paper. It is still an open question if and how these new materials will eventually be adopted into a silicon photonics foundry process for mass production. We foresee continuous progress and breakthroughs in this active research field.

\section{Silicon photonic devices for fiber-optic multiplexing}

As indicated by Eq. (1), the transmission capacity can be doubled using two orthogonal states of polarization and be scaled linearly by increasing spectral and spatial channels, leading to intensive studies of optical multiplexing technologies, including PDM, WDM, and, more recently, SDM. Hybrid multiplexing combining all these techniques is regarded as the only approach to boosting the data rate to $\mathrm{Pb} / \mathrm{s}$ level. A series of $\mathrm{Pb} / \mathrm{s}$ transmission experiments utilizing hybrid multiplexing technologies have been carried out in recent years [17-19, 191, 192]. In the study by Rademacher et al. [20], a record transmission capacity of $10.66 \mathrm{~Pb} / \mathrm{s}$ over $13 \mathrm{~km}$ was achieved with 114 spatial multiplicities through a 38-core three-mode fiber using $C+L$ bands. Among various multiplexing and demultiplexing techniques, silicon-based (de)multiplexers have attracted a tremendous interest because of their ultracompact footprints, decent performance, and low cost. They are expected to play a critical role in next-generation fiber-optic communication systems. PDM using silicon PSRs is already adopted in commercial monolithically integrated dualpolarization coherent transceivers [82].

A variety of (de)multiplexers have been proposed on SOI in the past several years [193-195]. In this section, we review recent progress in SOI-based (de)multiplexing technologies. In Section 4.1, we review the PDM devices on the SOI platform, which double the channel counts in a cost-effective way. In Section 4.2, we review wavelength (de)multiplexers with a focus on dense WDM (DWDM) that exploits tens or hundreds of wavelengths to augment the overall throughput. In addition to PDM and WDM, we have seen impressive demonstrations of SDM on silicon for multimode and multicore fibers to overcome the capacity limit of single-mode transmission, which will be reviewed in Section 4.3.

\subsection{Polarization splitters and rotators}

PDM can be realized using polarization-orthogonal modes in silicon waveguides, i.e., transverse electric (TE) and transverse magnetic (TM) modes. For instance, the capacity of an 8-channel WDM system can be effectively doubled by using a hybrid WDM-PDM (de)multiplexer with dual polarizations [196], as shown in Figure 14. A variety of polarization multiplexing circuits have been proposed in recent years [197] using either polarization beam splitters $[198,199]$ or PSRs. A PSR is often preferred in practical applications because, in addition to splitting the polarizations, it also converts one of the polarization (usually TM to TE) so that the rest of functional devices (such as modulators and PDs) can all work in the same polarization, eliminating the need for an additional set of designs for the

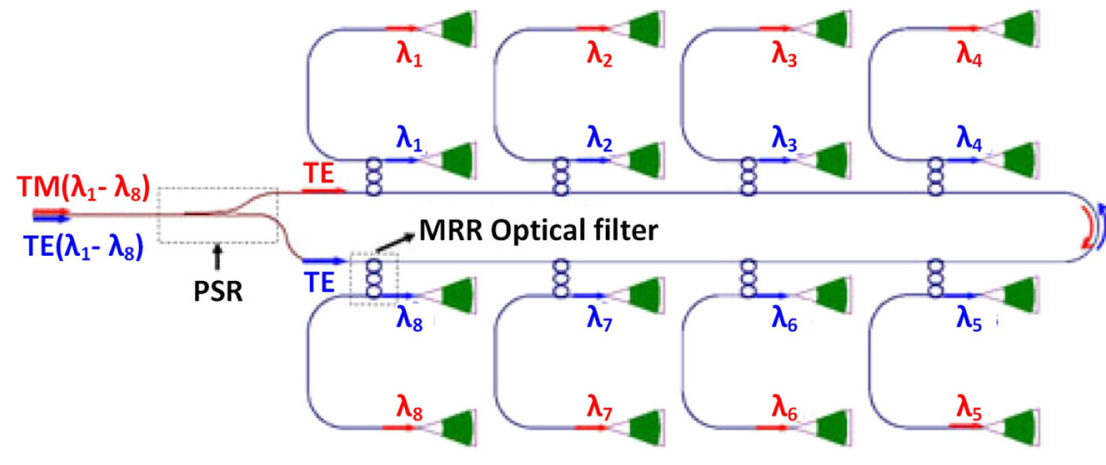

Figure 14: Schematic of a 16-channel hybrid WDM-PDM (de)multiplexer [196]. 


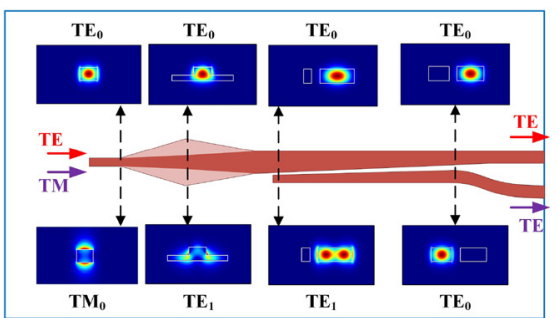

(a)

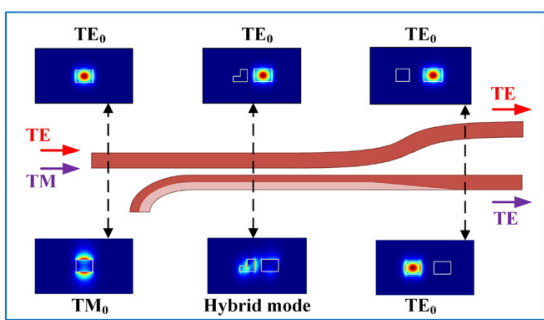

(b)
Figure 15: Schematics of two types of PSRs. (a) higher-order mode conversion: $\mathrm{TM}_{0}$ is firstly converted to $\mathrm{TE}_{1}$ using a bilevel taper and then to $\mathrm{TE}_{0}$ through adiabatic coupling; (b) hybrid-mode coupling: $\mathrm{TM}_{0}$ is converted to $\mathrm{TE}_{0}$ through the evolution of a hybrid mode. PSRs, polarization splitter-rotators; TE, transverse electric; TM, transverse magnetic. other polarization. At the transmitter side, the PSR is used as the polarization combiner in the reciprocal way, where the modulators can be designed in the polarization having the largest light-matter interaction and thus the highest efficiency (usually TE on submicron SOI).

To separate and rotate the fundamental TM mode, there are commonly two approaches: (1) higher-order mode conversion, where the input $\mathrm{TM}_{0}$ is firstly converted to the first-order quasi-TE $\left(\mathrm{TE}_{1}\right)$ mode that is then converted to fundamental $\mathrm{TE}\left(\mathrm{TE}_{0}\right)$ using structures such like $Y$ branches, MMI, or adiabatic couplers (ACs) [200-202]; (2) hybrid mode coupling, where the input $\mathrm{TM}_{0}$ is directly coupled into a hybrid mode (partial TE and partial TM) that is then converted adiabatically into TE [203-205]. The principles of these two approaches are illustrated in Figure 15(a) and (b), respectively. Both of them require a structure with an asymmetric refractive index profile in the vertical direction so that the fundamental TM can be effectively rotated. For strip waveguides, a simple way to break the vertical waveguide symmetry is to use an upper air cladding [206, 207]. However, a top cladding of silicon dioxide for protection is generally preferred in silicon industry packaging.

PSRs based on partially etched waveguides have been developed without requiring a clad other than $\mathrm{SiO}_{2}$. An experimental demonstration of PSRs compatible with standard foundry processes was presented by Guan et al. [203] in 2014. This PSR is based on a partially etched DC with a compact length of $27 \mu \mathrm{m}$. A low mode conversion loss of $0.5 \mathrm{~dB}$ and a low crosstalk of $-20 \mathrm{~dB}$ were observed over a wavelength regime from 1540 to $1570 \mathrm{~nm}$. In the same year, Sacher et al. [202] demonstrated a PSR using a bilevel tapered mode converter and an adiabatic coupler. The tested device has a crosstalk of less than $-13 \mathrm{~dB}$ over a bandwidth of $50 \mathrm{~nm}$. They also improved the crosstalk to $-22 \mathrm{~dB}$ over a bandwidth of $80 \mathrm{~nm}$ by integrating cascaded polarization filters. In 2017, Tan et al. [204] demonstrated an ultrabroadband PSR supporting $O-, C$-, and $L$-band operation. They verified the performance of the fabricated PSR within wavelength range of 1240-1360 and 1520-1620 nm, with the mode conversion efficiency of above $-0.97 \mathrm{~dB}$ and crosstalk of better than $-12 \mathrm{~dB}$. In 2018, Guo and Chu [208] demonstrated PSRs for both 1550 and $1310 \mathrm{~nm}$ using an optimized bilevel taper and a ridgewaveguide coupler to shrink the coupling length, showing an insertion loss of less than $1 \mathrm{~dB}$ and a crosstalk of less than $-20 \mathrm{~dB}$ within a $80-\mathrm{nm}$ bandwidth. We summarize some experimentally demonstrated PSRs with $\mathrm{SiO}_{2}$ cladding in the Table 3.

\subsection{Wavelength (de)multiplexers}

To increase the aggregate bandwidth, WDM is widely used in optical transmission systems [209]. As per the standard ITU G.694.1, a DWDM system can scale up to 160 channels with a channel spacing of $50 \mathrm{GHz}$ or even $25 \mathrm{GHz}$ [210]. Optical filter structures widely studied on silicon for DWDM

Table 3: Survey of PSRs on SOI with $\mathrm{SiO}_{2}$ cladding near $1550 \mathrm{~nm}$.

\begin{tabular}{|c|c|c|c|c|c|c|}
\hline Ref. & Year & Structure & Crosstalk (dB) & Insertion loss (dB) & Bandwidth $^{\mathrm{a}}(\mathrm{nm})$ & Length $(\mu \mathrm{m})$ \\
\hline [202] & 2014 & Bilevel MC $+\mathrm{AC}$ & -13 & 1.6 & 50 & 475 \\
\hline [203] & 2014 & Partially etched DC & -20 & 0.5 & 30 & 27 \\
\hline [204] & 2017 & Partially etched DC & -12 & 0.97 & 220 & 700 \\
\hline [205] & 2019 & Partially etched DC & -18 & 1.45 & 80 & 129 \\
\hline [208] & 2018 & Bilevel $M C+A C$ & -20 & 0.74 & 100 & 130 \\
\hline [104] & 2019 & Bilevel MC + AC & -20 & 1.5 & 85 & 375 \\
\hline
\end{tabular}

${ }^{a}$ Bandwidth is defined by the spectral range within which both the claimed crosstalk and insertion loss are achieved. PSRs, polarization splitter-rotators; MC, mode converter; AC, adiabatic coupler; DC, directional coupler; SOI, silicon-on-insulator. 
applications include arrayed waveguide gratings (AWGs) [211-214], echelle gratings (EGs) [215], cascaded MZIs [216], microring resonators (MRRs) [163, 217, 218], and Bragg grating filters such as contradirectional couplers [176, 219]. AWGs, EGs, and cascaded MZIs are classified as finite impulse response (FIR) filters, which split input light to dispersive paths and recombine them at different outputs with regard to wavelength. Usually, SOI-based multiplexers with a smaller channel spacing require longer and more delay lines. However, they are also more vulnerable to phase errors and losses [212, 220, 221]. While the channel spacing (determined by the group index) can be well controlled through a calibrated process, the alignment of center wavelengths to the ITU grid requires compensation for fabrication errors and temperature drift via, e.g., postfabrication trimming or thermo-optic tuning [222]. MRRs and Bragg gratings belong to infinite impulse response (IIR) filters. They are extremely compact [221] but suffer from sensitivity to fabrication errors and temperature variations. In contrast to AWGs and EGs, the center wavelength of each channel requires individual tuning and stabilization.

\subsubsection{FIR filters}

Recently reported SOI-based FIR filters are summarized in Table 4. AWGs outperform for a smaller channel spacing, while EGs are mostly used for multiplexing of fewer channels with a larger spacing. Different than AWGs, EGs implement reflective facets in a slab area to disperse and refocus light. Since the slab waveguide has significantly lower dispersion and group index compared to the waveguides used in an AWG, the delay length of EGs needs to be larger to obtain the same free spectral range (FSR) compared to AWGs, which causes a faster increase in footprint when many delay lines are needed. Besides, high- performance EGs also require high-quality reflecting facet, which is vulnerable to the thickness variation of waveguides [220]. In the study by Pommarede et al. [215], an EG with 16 channels and a 100-GHz spacing is presented on a 300-nm-thick SOI wafer, where Bragg mirrors are utilized to enhance the efficiency and the tolerance to fabrication variations.

Cascaded MZI filters route light through series of $2 \times 2$ MZIs, which stand for the wavelength splitter on each stage. Although their performance can be theoretically improved by cascading more MZI filters, the uncertainty in coupling coefficients and optical delays at each stage leads to increased crosstalk [221]. Therefore, postcorrection of phase errors is usually required. In the study by Munk et al. [216], photosensitive $\mathrm{As}_{2} \mathrm{Se}_{3}$ chalcogenide thin films deposited on top of silicon waveguides are used to compensate for fabrication errors in a cascaded MRR-assisted MZI filter; a narrow spacing of $17 \mathrm{GHz}$ has been achieved. Postfabrication phase trimming using germanium implanted waveguides is another means for fine tuning [223, 224]. In the study by Chen et al. [223], highaccuracy phase control was realized using a scanning laser that sequentially annealed sections of the implanted waveguide, by which an effective index change of 0.19 was achieved. However, as the footprint and complexity of cascaded MZIs scale quickly as the channel count increases, their suitability for massive DWDM channels remains questionable.

AWGs are among the most promising candidates for high-density wavelength multiplexing with massive channels. Several AWGs with a $25-\mathrm{GHz}$ spacing have been demonstrated on the SOI platform [211, 213]. In the study by Cheung et al. [213], a $512 \times 512$ silicon AWG router was demonstrated, as shown in Figure 16, covering $S, C$, and $L$ bands. Considering the high spectral density and ultrawide FSR, its footprint is actually quite compact $(11 \times 16 \mathrm{~mm}$ for

Table 4: Brief survey of FIR filters demonstrated on SOI.

\begin{tabular}{|c|c|c|c|c|c|c|}
\hline Ref. & Year & Type & Channel spacing (GHz) & Channel number & Crosstalk (dB) & Feature \\
\hline [215] & 2017 & EG & 100 & 16 & -15 & $\begin{array}{l}\text { 300-nm-thick wafer } \\
\text { Bragg mirrors as reflector }\end{array}$ \\
\hline$[216]$ & 2019 & Cascaded MZI & 17 & 8 & -22 & $\begin{array}{l}\text { Postcorrection using } \mathrm{As}_{2} \mathrm{Se}_{3} \\
\text { Nested MRR for band shaping }\end{array}$ \\
\hline [213] & 2013 & AWG & 25 & 512 & -4 & $\begin{array}{l}1200 \text { arrayed waveguides } \\
\text { Footprint of } 11 \times 16 \mathrm{~mm}^{2}\end{array}$ \\
\hline [214] & 2017 & AWG & 1 & 11 & -15 & $\begin{array}{l}\text { Postcorrection via heaters } \\
\text { Footprint of } 1.1 \mathrm{~cm}^{2}\end{array}$ \\
\hline$[225]$ & 2015 & Interleaved AWG & 200 & 18 & -15 & $\begin{array}{l}\text { Bidirectional AWG } \\
\text { MZI-based interleaver }\end{array}$ \\
\hline
\end{tabular}

AWG, arrayed waveguide grating; FIR, finite impulse response; SOI, silicon-on-insulator; EG, echelle grating; MZI, Mach-Zehnder interferometer; MRR, microring resonator. 
1200 arms). Due to the large channel count, the average delay length and phase errors rise up, leading to a relatively high crosstalk ( $-4 \mathrm{~dB})$. Similar to the study by Munk et al. [216], the performance can also be enhanced by phase error compensation. In the study by Gehl et al. [214], a record resolution of $1 \mathrm{GHz}$ is achieved by active phase correction using integrated thermo-optic PSs on the arrayed waveguides, at the cost of increased complexity and power consumption.

Another potential solution for a high-performance filter with massive channels is dual-stage or multistage filtering using a cascaded configuration $[226,227]$ or an interleaver [225, 228], whose principles are shown in Figure 17(a) and (b), respectively. For both approaches, the filter is disassembled to multiple components with less channels or lower resolution. Compared to the cascaded filters composing of a low-resolution primary filter and several high-resolution secondary filters, an interleaved structure offers a smaller footprint because only one highresolution interleaver is needed [228]. The major concern about an interleaved filter is the requirement for critically aligned central wavelengths for all secondary filters. In the study by Chen et al. [225], the authors proposed a multiplexer consisting of an MZI-based interleaver and a single bidirectional AWG (similar to an AWG router). The bidirectional AWG works equivalently as two unidirectional AWGs, enabling a halved channel spacing without doubling the footprint. It also eliminates the need for alignment between odd and even channels because they share the same arrayed waveguides connected to outputs of an MZI-based interleaver.

\subsubsection{IIR filters}

Serially coupled MRRs, often referred as microring coupled resonator optical waveguides, can achieve a flat response [229]. For DWDM applications, a large FSR is often needed to expand the total number of channels, along with a tunable filter response for channel allocation. Decreasing the ring radius is the most straightforward way to enlarge the FSR. With a tiny radius of $2.5 \mu \mathrm{m}$, a large FSR of $32 \mathrm{~nm}$ can be obtained [230]. For the tunability, microheaters are commonly used to change the effective index of silicon waveguides, by which the transmission spectrum of MRRs can be efficiently tuned. In the study by Bélanger-de Villers et al. [218], a thermally tunable filter in cascaded MRRs with

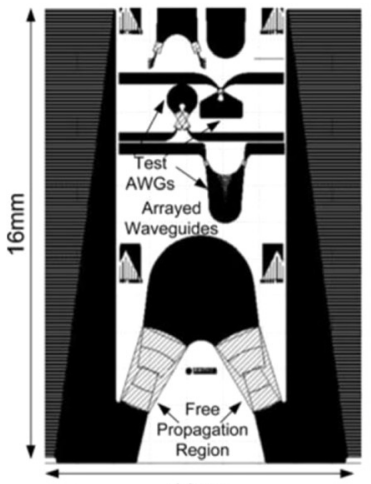

(a)

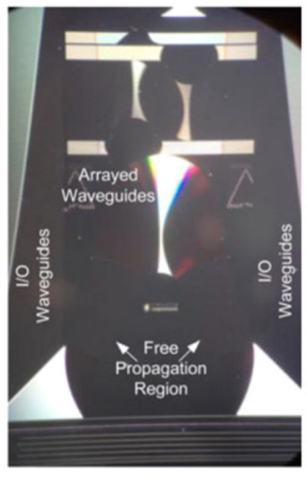

(b)

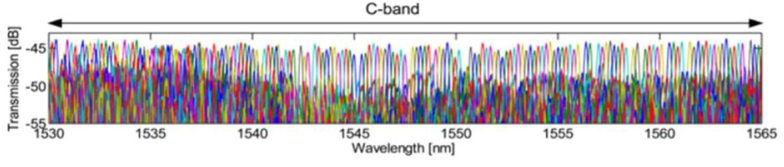

S-band

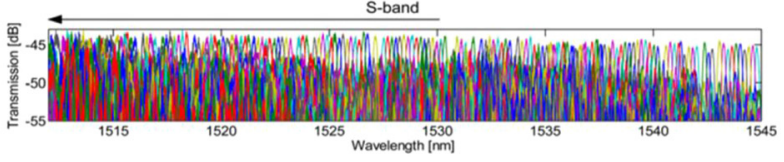

-ban

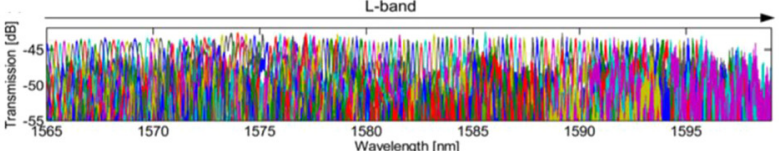

(c)

Figure 16: (a) Layout of $512 \times 512$ 25-GHz AWG. (b) Image of the fabricated device on the SOI platform. (c) Measured spectrum across the 512 channels, covering $S, C$, and $L$ bands [213]. AWG, arrayed waveguide grating; SOI, silicon-on-insulator.
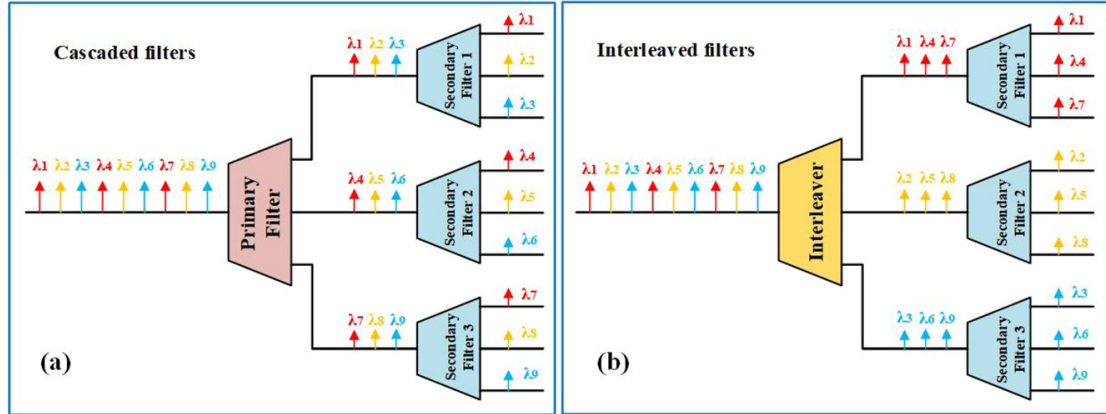

Figure 17: Schematic diagram of (a) cascaded filters and (b) interleaved filters. 


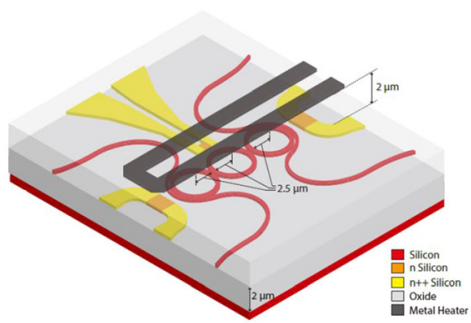

(a)

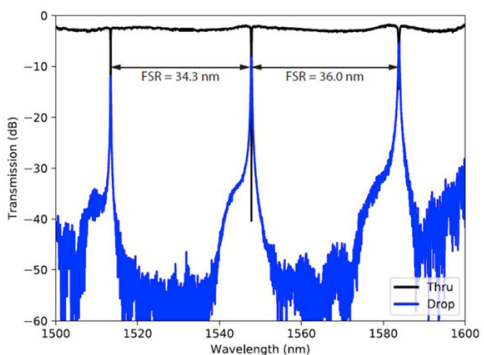

(b)

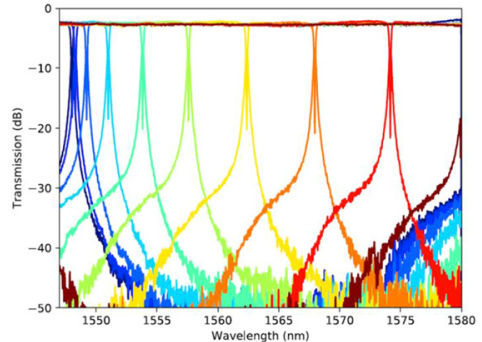

(c)

Figure 18: (a) Schematics of the cascaded microring filter; (b) transmission spectrum of the cascaded microring filter; (c) transmission spectrum of the cascaded microring under different applied power; [218].

(a)

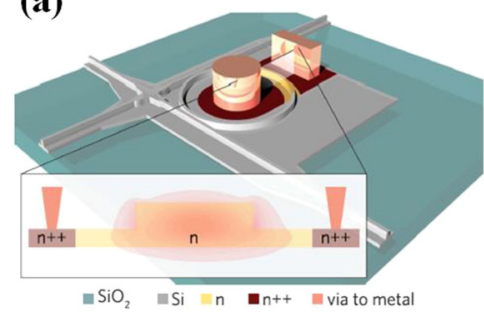

(b)

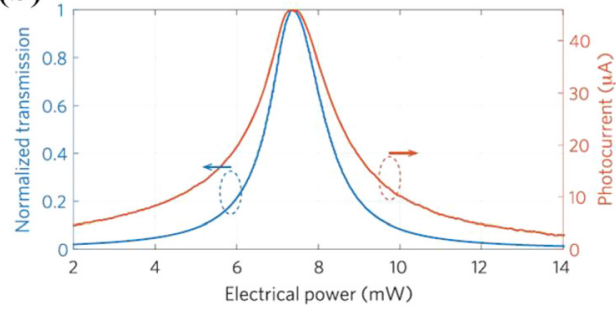

Figure 19: (a) Illustration of a photoconductive heater integrated into the MRR; (b) measured drop-port transmission (left axis) and photocurrent from the photoconductive heater (right axis) of the ring as a function of the supplied electrical power to the heater [234]. MRR, microring resonator. a small diameter and ultrawide FSR of $35 \mathrm{~nm}$ covering the entire C-band was demonstrated (Figure 18). The device consists of three serially cascaded rings with radii of $2.5 \mu \mathrm{m}$ and waveguide widths of $450 \mathrm{~nm}$. TiN heaters sit $2 \mu \mathrm{m}$ over the waveguides for simultaneous tuning of all the rings with a single control voltage. To precisely align the filter, an $\mathrm{N}$-doped silicon strip is placed close to each ring. By applying voltages to the heaters, the passbands can be redshifted over a $32 \mathrm{~nm}$ range with $120 \mathrm{~mW}$ power, as shown in Figure 18(c).

Silicon MRRs are sensitive to temperature variations due to the high thermo-optic coefficient of silicon $\left(1.86 \times 10^{-4} \mathrm{~K}^{-1}\right)$. They are also vulnerable to fabrication errors. For example, for a silicon microring with a waveguide width of $0.5 \mu \mathrm{m}$ and a height of $0.22 \mu \mathrm{m}$, a very small variation of $1 \mathrm{~nm}$ in the width would introduce a $0.8 \mathrm{~nm}$ $(100 \mathrm{GHz})$ shift in the resonant wavelength [231]. At the $130 \mathrm{~nm}$ node (200 $\mathrm{mm}$ wafers and $193 \mathrm{~nm}$ dry lithography), the $3 \sigma$ variation of the waveguide width and thickness is around 8 and $6 \mathrm{~nm}$, respectively [232]. It is thus crucial to precisely control the resonance for practical applications. For a few-MRR design, transmission spectra compensation via thermal tuning is relatively easy. However, the situation becomes complicated when hundreds of MRRs are integrated in a large-scale WDM system; hence, lowconsumption and robust solutions for resonance locking withstanding temperature variations are vitally important. Padmaraju and Bergman [233] provide a review on the thermal challenges for MRRs, in which both athermal solutions and control-based solutions are discussed in detail. Athermal solutions usually require adding an exotic material with a negative thermo-optic coefficient (e.g., $\mathrm{TiO}_{2}$ ). However, the hurdles in this solution lie in the difficulty of precisely tuning the thermo-optic coefficient and the incorporation with the standard fabrication process. An alternative way is embedding the MRR in a thermally matched MZI. This solution does not require any new materials and is compatible with current CMOS fabrication techniques. However, it also suffers from the susceptibility to fabrication errors and the footprint increasement of filters. Therefore, control-based solutions are generally preferred for large-scale integration of MRRs. Recently, Jayatilleka et al. [234] proposed a photoconductive heater toward automatic resonator locking for large-scale MRR circuits. Figure 19(a) illustrates the photoconductive heater integrated in an MRR. A lightly n-doped $\left(5 \times 10^{17} \mathrm{~cm}^{-3}\right)$ waveguide is embedded in the resonator, performing as a heater and power monitor concurrently. The doping creates interband defects in silicon for optical absorption and photocurrent generation. By sweeping the power applied onto the heater, a synchronization of intracavity optical power and photocurrent can be observed, as shown in Figure 19(b). Hence, the ring can be resonator locked automatically by maximizing the photocurrent [235]. This photocurrent heater allows individual rings to be precisely sensed and controlled simultaneously without any 
additional photodetector or fabrication steps, providing a low-cost solution for large-scale MRR-based multiplexing systems.

\subsection{Optical interfaces for SDM transmission}

Recently, the capacity of SMF has been boosted to $151 \mathrm{~Tb} / \mathrm{s}$ by using $400 \times 23.6$-GBaud WDM-PDM-256 QAM signal [236]. To break the capacity crunch, parallel SMFs have been currently used to substantially increase the transmission capacity of submarine systems, which is regarded as SDM 1.1 [37]. A "cost-per-bit cruch" is foreseen as the number of SMFs scales up, which creates a strong demand for disruptive technologies beyond the SMF-based SDM. In this context, MCFs, few-mode fibers (FMFs) or multimode fibers (MMFs), and few-mode multicore fibers (FM-MCFs) have been extensively studied, offering tens of spatial channels within a single strand. Channel numbers of 37 and 45 have been achieved using MCFs and MMFs, respectively [237, 238]. A large channel count exceeding 100 has been experimentally examined using FM-MCFs [239, 240]. Microscope photos of example MCFs and FM-MCFs are shown in Figure 20(a) and (b), respectively. However, achieving a larger channel count is challenging due to the requirement of a larger cladding diameter that induces instability of the fiber. On the other hand, multiplexing in orbital angular momentum (OAM) modes attracts much attention in recent years. Compared to linearly polarized (LP) modes, OAM modes are formed by eigenmodes with potentially lower interactions and thus lower cross talk, which facilitates a transmission system requiring less complex DSP. Many OAM fibers were designed and demonstrated during the past years [241243]. Figure 20(c) shows an OAM fiber that supports 36 OAM states (OAM order from -9 to 9 in two orthogonal states of polarization) [243]. For transmitting information from chips to these specially crafted fibers, novel optical interfaces are inevitable. These interfaces are usually composed of a number of grating couplers or 3D waveguides, coupling light to different cores or fiber modes. In this section, we review the recent process in silicon photonic interfaces for MMFs (LP modes and OAM modes) and MCFs.

\subsubsection{Optical interfaces for LP mode (de)multiplexing}

Most reports on LP modes (de)multiplexers use grating couplers (GCs) as the interface, taking advantages of flexible arrangement for mode shaping and multiplexing. The general principle is to project single-mode inputs onto the fiber modes through on-chip linear optical functions, which can be described using the transfer matrix method:

$$
\mathbf{E}_{o}=\mathbf{M}_{C} \mathbf{M}_{L} \mathbf{E}_{i}
$$

where $\mathbf{E}_{i(o)}=\left[E_{i(o), 1}, E_{i(o), 2 \ldots}\right]^{\prime}$ is the amplitude vector of the input (output) modes and $\mathbf{M}_{L}$ and $\mathbf{M}_{C}$ are the transfer matrices for the on-chip linear operation and coupling coefficients at the optical interface, respectively. In the ideal case of a perfect one-to-one mode conversion procedure, the product of $\mathbf{M}_{C}$ and $\mathbf{M}_{L}$ should be an identity matrix. In practice, its nondiagonal elements represent mode crosstalk.

The on-chip linear operation $\mathbf{M}_{L}$ can be realized using a number of waveguide couplers and PSs. For example, Figure 21(a) shows the schematic of a mode multiplexer based on a grating coupler array [244]. Light injected to $\mathrm{CH} 1$ is split into two beams, which reaches output ports 2 and 4 with a phase difference of $\pi$, thus exciting $\mathrm{LP}_{11-a y}$. Similarly, a total of $8 \mathrm{LP}$ modes can be generated, six of which can be clearly observed as shown in Figure 21(b) since the FMF used in experiment does not support $\mathrm{LP}_{21}$ modes. By employing an aluminum mirror, a coupling efficiency of $-10.6 \mathrm{~dB}$ with $3.7 \mathrm{~dB}$ mode-dependent coupling loss is achieved. In the study by Tong et al. [245], a single 2D grating coupler was used to multiplex modes into FMF, as depicted in Figure 21(c). Injecting $\mathrm{TE}_{0}$ mode to the $\mathrm{LP}_{01-y}$ launching port generates the $\mathrm{LP}_{01}$ mode at the output interface. If $\mathrm{TE}_{0}$ mode is fed into the $\mathrm{LP}_{11-y}$ launching port, the fundamental mode will be converted to $\mathrm{TE}_{1}$ mode and $\mathrm{LP}_{11-y}$ mode, instead. Similarly, $\mathrm{LP}_{01-x}$ and $\mathrm{LP}_{11-x}$ modes can be generated as light is injected to corresponding launching ports. The
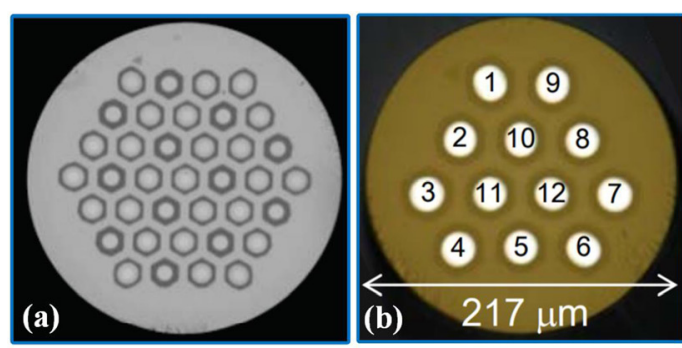

Figure 20: Optical microscope photo of (a) MCF with single-mode 37 cores [237]; (b) FM-MCF with 12 hexagonally arranged 10-mode cores [240]; (c) OAM fiber supporting 36 OAM states [243]. MCF, multicore fibers; FM-MCF, few-mode multicore fiber; OAM, orbital angular momentum. 


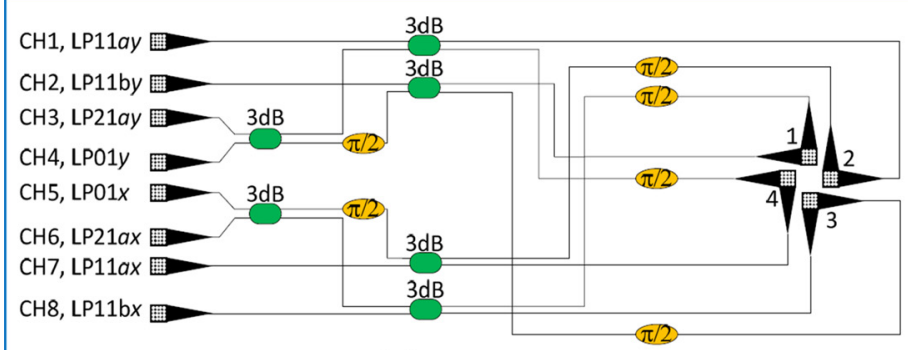

(a)

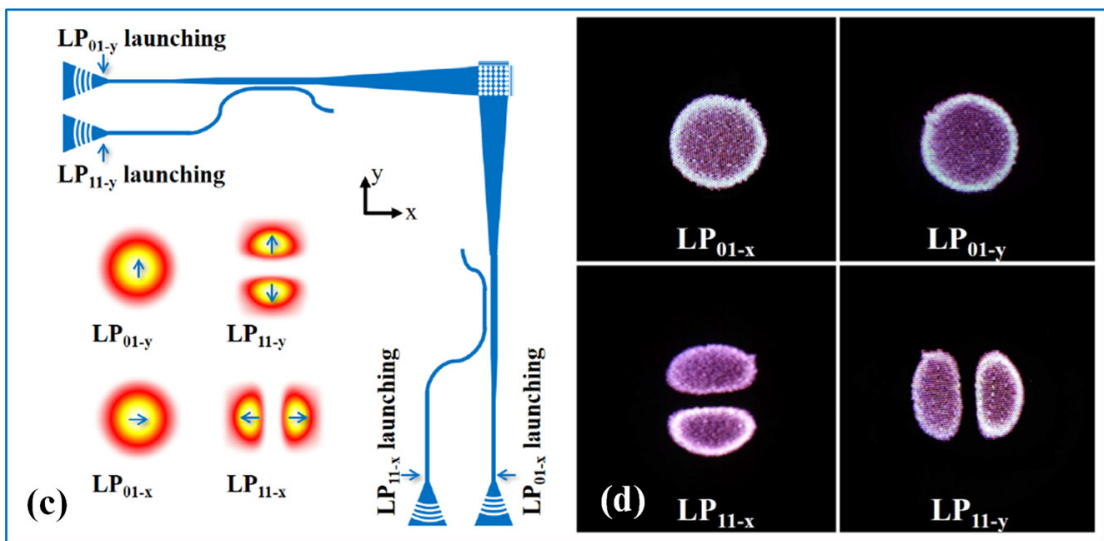

Figure 21: (a) Schematic of a mode multiplexer using a grating coupler array and (b) its generated modes observed after transmission through a 5-m long FMF that supports only $L P_{01}$ and $L P_{11}$ modes [244]. (c) Schematic of a $2 \mathrm{D}$ grating coupler-based multiplexer and (d) its generated mode profiles measured after fiber transmission [245]. FMF, few-mode fiber.

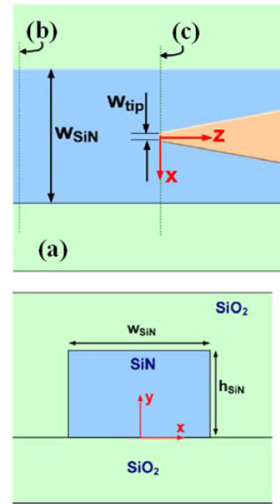

(b)

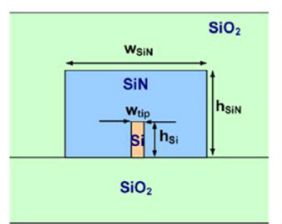

(c)

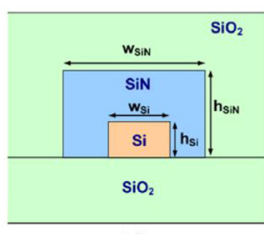

(d)
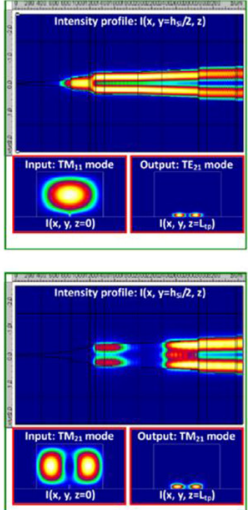
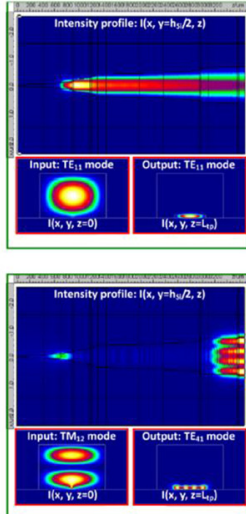

(e)
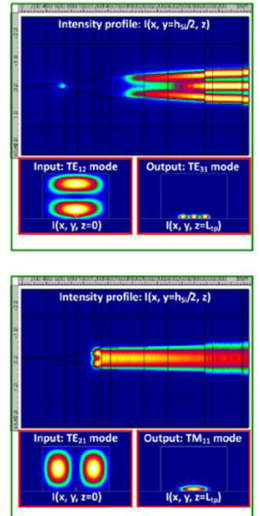

Figure 22: (a) Schematic of mode convertor; (b)-(d) cross section of a convertor at different locations; (e) simulated light propagation showing the mode conversion [246].

experimental results are shown in Figure 21(d). In this work, grating structures are optimized with genetic optimization algorithm, thus a coupling efficiency of $-4.9 \mathrm{~dB}$ with $1.2 \mathrm{~dB}$ mode-dependent loss of is achieved.

There are few reports on multiplexers using edge coupling, as the submicron-thick silicon wafer usually does not support higher-order modes in the vertical direction. However, GCs have a relatively narrow bandwidth (typically $30-50 \mathrm{~nm}$ ) and, therefore, side coupling is indispensable for ultrawide-band operation. In the study by Dai and Mao [246], they proposed a multimode convertor using a SOI inverse taper buried in a $3 \times 3 \mu \mathrm{m}^{2}$ multimode SiN strip waveguide, as shown in Figure 22, where six modes in the SiN waveguide (analogous to the $\mathrm{LP}_{01}, \mathrm{LP}_{11 a}, \mathrm{LP}_{11 b}$ modes in $x$ and $y$ polarizations of a FMF) can be respectively coupled to different modes of a multimode silicon waveguide (simulation results are shown in Figure 22(e)). Although the number of modes is limited by the geometry of the SiN waveguide, this technology is promising for FMF transmission. 
Thanks to the rapid development of heterogeneous 2D/ 3D photonic integration technology [247], SOI-PICs can be efficiently connected to 2D/3D waveguides on other material platforms via optical wire bonding [248] or interlayer coupling [249]. This opens the possibility of mode multiplexers via flexible polymer and glass waveguides among others [250-253]. 3D PICs can be formed by either ultrafast laser inscription or multilayer stacking. In the study by Gross et al. [250], a three-core asymmetric mode-selective coupler was fabricated using the femtosecond laser directwrite technique, multiplexing $\mathrm{LP}_{11 a}$ and $\mathrm{LP}_{11 b}$ modes across a wavelength range exceeding $C$ band, as illustrated in Figure 23(a). Similarly, a multilayer asymmetric coupler was fabricated with polymer materials, showing high coupling ratios of above $90 \%$ for the two $\mathrm{LP}_{11}$ modes, as shown in Figure 23(b) [251]. Taking advantage of edge coupling, both the couplers mentioned above have relatively low insertion loss, low wavelength sensitivity, and low polarization dependency, promising for multidimensional multiplexing in the near future.

\subsubsection{Optical interfaces for OAM modes (de) multiplexing}

OAM modes are characterized by helical phase fronts that can be obtained by MRRs with periodically coupled emitters [254-257], superposed holographic fork gratings [258], and star couplers [259-262]. OAM generators based on MRRs are ultracompact and can be flexibly engineered for high-order OAM. However, in these structures, OAM beams are generated at resonances, where the topological charge depends on the resonator's longitudinal mode [254-257]. As a result, they are wavelength selective and, therefore, generally not compatible with WDM transmission. In the study by Zhou et al. [258], the authors proposed a
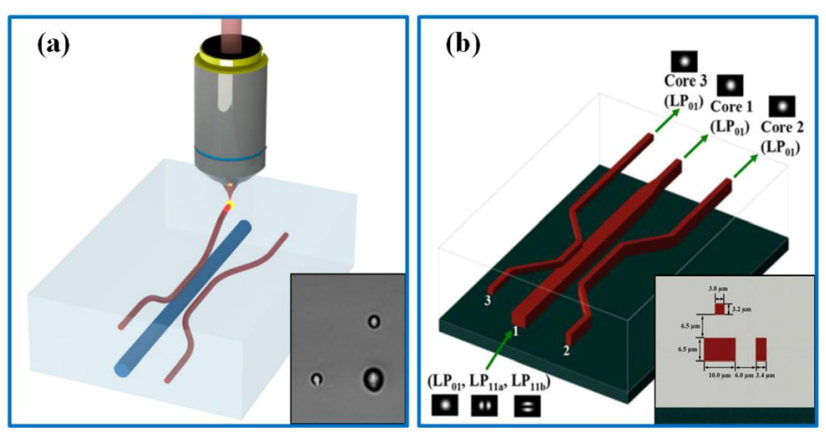

Figure 23: (a) Schematic of a laser-write 3D coupler for $\mathrm{LP}_{11 a}$ and $\mathrm{LP}_{11 b}$ multiplexing; insert: end-face microscope image of the threecore coupler [250]. (b) Schematic of a multilayer asymmetric coupler for $\mathrm{LP}_{11 a}$ and $\mathrm{LP}_{11 b}$ multiplexing; insert: cross section of the threecore coupler [251]. broadband OAM generator based on a 2D subwavelength surface (superposed holographic fork gratings). The generator has an ultracompact footprint of $3.6 \times 3.6 \mu \mathrm{m}^{2}$, by which 4 OAM modes $\left(x\right.$ - $/ y$-polarized $\left.\mathrm{OAM}_{+1} / \mathrm{OAM}_{-1}\right)$ can be generated from 1500 to $1630 \mathrm{~nm}$. It will be interesting to see if this method can support a larger number of OAM modes. Alternatively, in the study by Fontaine et al. [259], the authors proposed a scalable OAM generator using a star coupler and a circular grating coupler and experimentally observed 9 OAM states.

The state of polarization is essential for transmission via OAM fibers that carries modes in circular polarization states [263]. Most OAM generators demonstrated on silicon are in cylindrical polarization (either azimuthal or radial) $[254,256,257,259,264]$. Using two star couplers and 2D antennas, a circularly polarized OAM generator/multiplexer was proposed [261] and experimentally demonstrated [262]. The block diagram is illustrated in Figure 24(a). By injecting signals to the upper or lower star coupler, OAM modes with left circular polarization state or right circular polarization state can be excited, respectively. A DC array is used to control the polarization state of OAM, by which a circular polarization can be achieved at output. A series of 2D grating couplers are arranged in a circle matching the mode profile with a ring-core OAM fiber [243]. OAM orders ranging from -3 to +3 are successfully achieved, in both left and right circular polarization states, as shown in Figure 24(d).

Table 5 summarizes some of the experimentally demonstrated mode-division multiplexing (MDM) (LP and OAM) on the SOI platform. We can see that the insertion losses of these devices are very high and should be improved by optimization in design and fabrication.

\subsubsection{Optical interfaces for multicore transmission}

Compared to MMFs, single-mode MCFs have no or less requirement for multiple-input multiple-output DSP and, therefore, may be adopted earlier in real applications. They also provide an effective means of dense fiber-to-chip integration because they offer a small spacing and twodimensional arrangement for fiber-to-chip couplers. Using Si-PICs and MCF, several SDM links have been demonstrated in the past few years. A chip-to-chip interconnect with a capacity of $80 \mathrm{~Gb} / \mathrm{s}(4 \times 20 \mathrm{~Gb} / \mathrm{s})$ was presented in 2017, using 9 cores of a 19-core MCF (as illustrated in Figure 25(a)), alongside plasmonic modulators and germanium photodetectors [265]. Later on, an aggregate capacity of $100 \mathrm{~Gb} / \mathrm{s}$ over $1 \mathrm{~km}$ was demonstrated using 4 cores of an MCF with $25 \mathrm{~Gb} / \mathrm{s}$ modulation, as shown in Figure 25(b) [266]. Also in 2017, De Heyn et al. [267] 

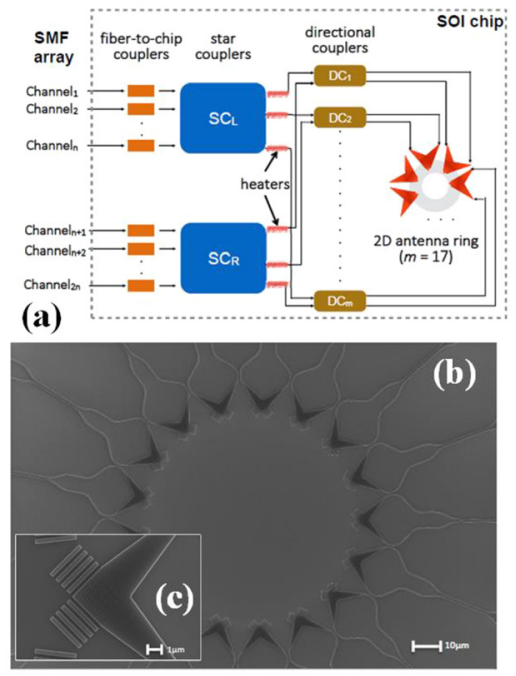

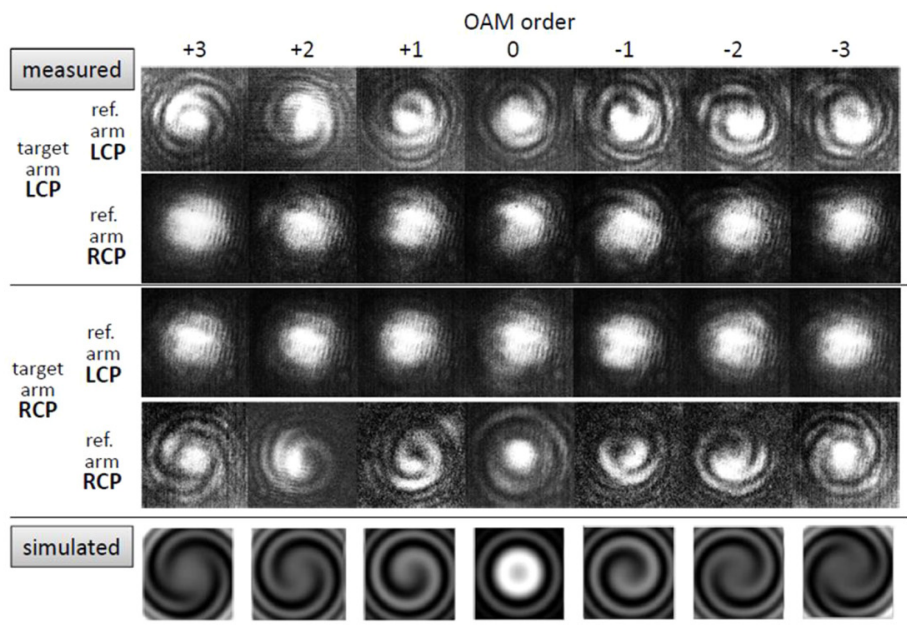

(d)

Figure 24: (a) Block diagram of an OAM generator/multiplexer; (b) Scanning eletron microscopy (SEM) image of the fabricated emitter section; (c) SEM image of a 2D grating coupler; (d) measured interferograms for different OAM orders [262]. OAM, orbital angular momentum.

Table 5: Survey of experimentally demonstrated multi-mode (de)multiplexers on SOI.

\begin{tabular}{llllrr}
\hline Ref. & Year & Structure & $\begin{array}{l}\text { Mode } \\
\text { type }\end{array}$ & $\begin{array}{r}\text { Channel } \\
\text { number }\end{array}$ & $\begin{array}{r}\text { Loss } \\
\text { (dB) }\end{array}$ \\
\hline$[244]$ & 2015 & GC array with back- & LP & 8 & $14.3^{\mathrm{a}}$ \\
& & side mirrors & & & \\
{$[245]$} & 2019 & 2D GC & LP & 4 & $6.1^{\mathrm{b}}$ \\
{$[258]$} & 2019 & Holographic GC & OAM & 4 & $8.27^{\mathrm{c}}$ \\
{$[259]$} & 2012 & SC + circular GC & OAM & 9 & - \\
{$[262]$} & 2019 & SC + GC array & OAM & 14 & $19.7^{\mathrm{c}}$ \\
\hline
\end{tabular}

${ }^{\mathrm{a}}$ Fiber-to-fiber.

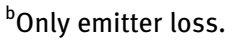

'On-chip loss and emitter loss.

OAM, orbital angular momentum; GCs, grating couplers; SOI, siliconon-insulator; SC, star couplers. demonstrated an MCF optical link with an aggregate capacity of $896 \mathrm{~Gb} / \mathrm{s}$. This 16-channel SDM system optical link utilizes a 37-core MCF coupled to a 2D array of grating couplers on a silicon chip where a dense array of $56 \mathrm{~Gb} / \mathrm{s}$ GeSi electroabsorption modulators and photodetectors are cointegrated, as shown in Figure 25(c).

Combining multimode and multicore techniques, FM-MCFs allow for an even higher capacity. GC-based solutions for LP or OAM (de)multiplexing take a relatively large footprint, making the mode matching a significant challenge for an FM-MCF that has a narrow core pitch of about $50 \mu \mathrm{m}$ [192]. In this case, optical interfaces via edge coupling may become crucial. A potential solution is to use hybrid integration with 3D waveguides. For instance, a monolithic FM-MCF multiplexer based on 3D waveguides was proposed in the study by Riesen et al. [268],

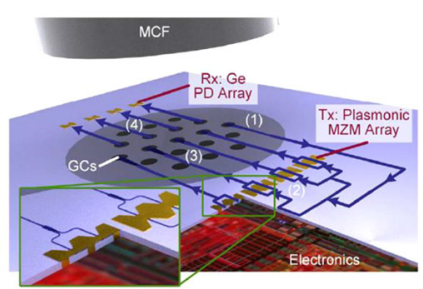

(a)

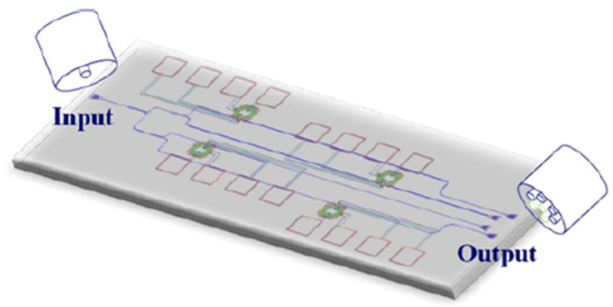

(b)

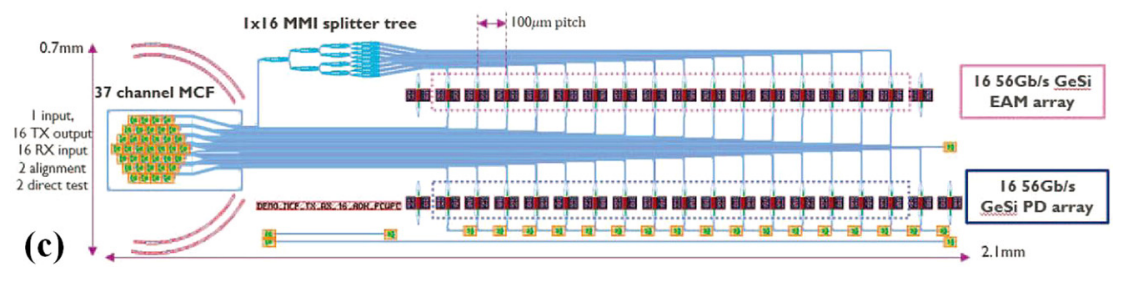

Figure 25: (a) Schematic of an $80 \mathrm{~Gb} / \mathrm{s}$ optical interconnect where 9 cores of a 19-core fiber were utilized: one core for CW input, 4 cores for transmitter outputs, and 4 for receiver inputs [265]. (b) Illustration of a silicon photonic SDM transmitter for $100 \mathrm{~Gb} / \mathrm{s}$ 4-channel MCF transmission [266]. (c) Schematic of a 16-channel transceiver with a capacity of $896 \mathrm{~Gb} / \mathrm{s}$ through 37 cores of a 61-channel fiber array: one core for CW input, 32 for transmitter outputs and receiver inputs, 2 for reference test, and 2 for alignment [267]. CW, continuous wave; SDM, space-division multiplexing; MCF, multicore fiber. 


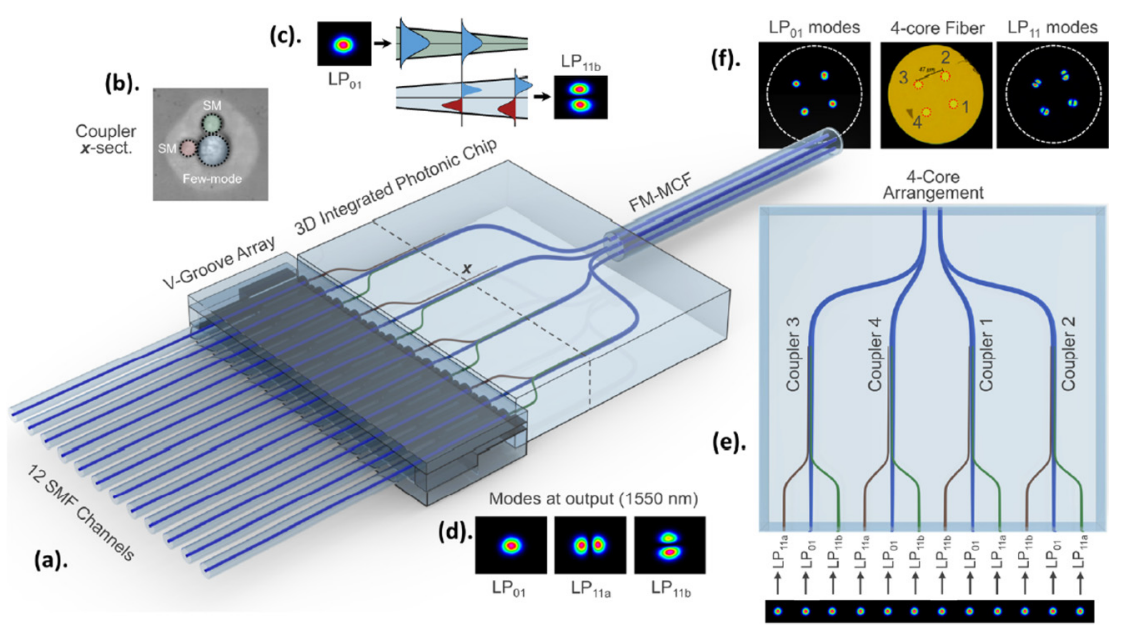

Figure 26: (a) 3D schematic of FM-MCF multiplexer. (b) Microscope image of cross section of few-mode coupler; (c) the adiabatic mode evolution in few-mode coupler; (d) infrared images of $\mathrm{LP}_{01}, \mathrm{LP}_{11 a}$, and $\mathrm{LP}_{11 b}$ modes of one core. (e) Top view of multiplexer; (f) cross section of a 3-mode 4-core fiber and modes excited in each core [268]. FM-MCF, few-mode multicore fiber. which addresses multiple SMFs, each coupled to a designated LP mode of one core of an FM-MCF fiber, as shown in Figure 26. In this study, 3 modes $\times 4$ cores were excited over $S+C+L$ band, with a low insertion loss of $1.8 \mathrm{~dB}$. Meanwhile, the device exhibits a high mode extinction ratio of around $22 \mathrm{~dB}$, along with a low intercore crosstalk of less than $-28 \mathrm{~dB}$. In addition, the multiplexing capability can be scaled in an increasing number of modes or cores. Considering its broad bandwidth, low loss, and volume scalability, such a hybrid integration strategy is attractive for future development of SDM systems.

\section{Conclusion}

The demand for low-cost, integrated solutions in optical communications has been a major driving force behind the emergence and rapid development of silicon photonics. This disruptive technology will continue evolving at a fast pace in the next decade to support the scaling of capacity of fiber-optic transmission systems. In the next few years, commercial $\mathrm{Tb} / \mathrm{s}$ silicon photonic transceivers will be available for various distances ranging from hundreds meters to thousands of kilometers for a vast diversity of application scenarios including intradata and interdata center interconnects, $5 \mathrm{G}$ access networks, terrestrial optical transport networks, and submarine cables. Silicon photonics will play an essential role in enabling massive WDM-SDM multiplexing by providing high-density, lowcost, and low-power optoelectronic functions (such as modulators, PDs, and VOAs) and compact wavelength and spatial multiplexing solutions.

Silicon photonics still faces severe challenges. Toward 100+ Gbaud transceivers, innovations in driving configuration and CMOS-photonics cointegration are needed to break the trade-off between modulation efficiency and bandwidth in silicon modulators. Densely integrated electronic-photonic circuits are essential for massively parallel transmission systems to realize the full potential of the WDM $\times$ SDM paradigm. With the everincreasing demands for bandwidth density and energy efficiency, commercial systems may eventually adopt novel photonic structures (e.g., resonators and slow-light modulators) and heterogeneously integrated devices using materials such as III-V compounds, $\mathrm{LiNO}_{3}$, plasmonic, polymer, graphene, etc. Instead of competing with silicon, these materials will more likely be incorporated onto the silicon platform and enrich the silicon photonics library. The performance of silicon multiplexers for DWDM is inferior to their counterparts on traditional platforms such as glass planar lightwave circuits. Their advantages lie in the prospect of monolithic integration with other active and passive functions. Owing to its extreme flexibility and wide accessibility, silicon photonics provides a unique platform for the exploration of novel concepts such as multiplexers for novel SDM fibers and systems.

Some silicon photonic devices have not been covered by this review but are also crucial for next-generation highcapacity tranmission systems. On-chip lasers and amplifiers via heterogeneous integration are under active development [190, 269]. Microresonator-based optical frequency combs have shown a great potential for multiwavelength sources in massively parallel optical communications [270-272]. Optical switching without the optical-electrical-optical conversion may enable highefficiency, low-cost optical networks. Large-scale integrated optical switches have been demonstrated in Si-PICs using thermo-optic [273, 274], electrostatic [275], and electro-optic [276] effects. 
Acknowledgment: The authors acknowledge the Natural Sciences and Engineering Research Council of Canada for the research grant RGPIN-2020-06382. The authors thank Drs. Jiachuan Lin and Zhiping Jiang with Huawei Canada for useful discussions.

Author contribution: All the authors have accepted responsibility for the entire content of this submitted manuscript and approved submission.

Research funding: This research was funded by the Natural Sciences and Engineering Research Council of Canada for the research grant RGPIN-2020-06382.

Conflict of interest statement: The authors declare no conflicts of interest regarding this article.

\section{References}

[1] P. J. Winzer, D. T. Neilson, and A. R. Chraplyvy, "Fiber-optic transmission and networking: the previous 20 and the next 20 years," Opt. Express, vol. 26, no. 18, pp. 24190-24239, 2018.

[2] D. J. Richardson, "Filling the light pipe," Science, vol. 330, no. 6002, pp. 327-328, 2010.

[3] 2020 Wikipedia, May [Online]. Available at: https://en. wikipedia.org/wiki/Internet_traffic.

[4] P. J. Winzer and D. T. Neilson, "From scaling disparities to integrated parallelism: A decathlon for a decade," J. Lightwave Technol., vol. 35, no. 5, pp. 1099-1115, 2017.

[5] 2020 Sandvine Updates, May [Online]. Available at: https:// www.sandvine.com/covid-19-trends.

[6] Cisco annual internet report (2018-2023). [Online]. Available at: https://www.cisco.com/c/en/us/solutions/executiveperspectives/annual-internet-report/index.html.

[7] K. Kikuchi, "Fundamentals of coherent optical fiber communications," J. Lightwave Technol., vol. 34, no. 1, pp. 157-179, Jan 2016.

[8] E. Agrell, M. Karlsson, A. Chraplyvy, et al., "Roadmap of optical communications," J. Opt., vol. 18, no. 6, p. 063002, 2016.

[9] F. Kish, R. Nagarajan, D. Welch, et al., "From visible lightemitting diodes to large-scale III-V photonic integrated circuits," Proc. IEEE, vol. 101, no. 10, 2013, pp. 2255-2270.

[10] B. Glover, History of the Atlantic Cable Undersea Communications, 2020 [Online]. Available at: https://atlanticcable.com/Cables/CableTimeLine/index2001.htm.

[11] V. Vusirikala, A Quick Hop across the Pond: Supercharging the Dunant Subsea Cable with Sdm Technology, 2019 [Online]. Available at: https://cloud.google.com/blog/products/ infrastructure/a-quick-hop-across-the-pond-superchargingthe-dunant-subsea-cable-with-sdm-technology.

[12] Huawei Launches Industry's First 800 G Tunable Ultra-highspeed Optical Module, 2020 [Online]. Available at: https://www. huawei.com/en/press-events/news/2020/2/800g-tunableultra-high-speed-optical-module.

[13] K. Fukuchi, T. Kasamatsu, M. Morie, et al., "10.92-Tb/s ( $273 \times 40-\mathrm{Gb} / \mathrm{s})$ triple-band/ultra-dense wdm optical-repeatered transmission experiment," in Optical Fiber Communication Conference, pp. PD24, Anaheim, Optical Society of America, 2001. https://doi.org/10.1364/OFC.2001.PD24.
[14] A. Sano, "14-Tb/s (140x111-Gb/s PDM/WDM) CSRZ-DQPSK transmission over $160 \mathrm{~km}$ using $7-\mathrm{THz}$ bandwidth extended L-band EDFAs," ECOC 2006 Proc., vol. 6, 2006.

[15] A. H. Gnauck, G. Charlet, P. Tran, et al., "25.6-Tb/s C + L-band transmission of polarization-multiplexed RZ-DQPSK signals," in Optical Fiber Communication Conference, pp. PDP19, Anaheim, Optical Society of America, 2007.

[16] B. Zhu, T. Taunay, M. Fishteyn, et al., "112-Tb/s space-division multiplexed DWDM transmission with $14-\mathrm{b} / \mathrm{s} / \mathrm{Hz}$ aggregate spectral efficiency over a 76.8-km seven-core fiber," Opt. Express, vol. 19, no. 17, pp. 16665-16671, 2011.

[17] H. Takara, A. Sano, et al., "1.01-Pb/s (12 SDM/222 WDM/456 Gb/ s) Crosstalk-managed Transmission with $91.4-\mathrm{b} / \mathrm{s} / \mathrm{Hz}$ Aggregate Spectral Efficiency," in European Conference and Exhibition on Optical Communication, Amsterdam, Optical Society of America, 2017, paper Th.3.C.1, https://doi.org/10.1364/ECEOC.2012.Th. 3.C.1.

[18] B. Puttnam, R. Luís, W. Klaus, et al., " $2.15 \mathrm{~Pb} / \mathrm{s}$ transmission using a 22 core homogeneous single-mode multi-core fiber and wideband optical comb," in 2015 European Conference on Optical Communication (ECOC), Valencia, IEEE, pp. 1-3 2015. https://doi.org/10.1109/ECOC.2015.7341685.

[19] D. Soma, Y. Wakayama, S. Beppu, et al., "10.16 peta-bit/s dense SDM/WDM transmission over low-DMD 6-mode 19-core fibre across C + L band," in 2017 European Conference on Optical Communication (ECOC), pp. 1-3, IEEE, Gothenburg, 2017. https://doi.org/10.1109/ECOC.2017.8346082.

[20] G. Rademacher, B. J. Puttnam, R. S. Luís, et al., " 10.66 peta-bit/s transmission over a 38-core-three-mode fiber," in Optical Fiber Communication Conference, p. Th3H-1, San Diego, Optical Society of America, 2020. https://doi.org/10.1364/OFC.2020. Th3H.1.

[21] X. Chen, M. M. Milosevic, S. Stanković, et al., "The emergence of silicon photonics as a flexible technology platform," Proc. IEEE, vol. 106, no. 12, 2018, pp. 2101-2116.

[22] A. Rahim, T. Spuesens, R. Baets, and W. Bogaerts, "Open-access silicon photonics: current status and emerging initiatives," Proc. IEEE, vol. 106, no. 12, 2018, pp. 2313-2330.

[23] “Simply silicon Editorial,” Nat. Photonics, vol. 4, p. 491, 2010.

[24] J. E. Bowers, R. Alferness, R. L. Clark, et al., "American institute for manufacturing integrated photonics (AIM photonics)," in Avionics and Vehicle Fiber-Optics and Photonics Conference, pp. 24-24, Santa Barbara, IEEE, 2015. https://doi.org/10.1109/ AVFOP.2015.7356648.

[25] M. Hochberg and T. Baehr-Jones, "Towards fabless silicon photonics," Nat. Photonics, vol. 4, p. 492, 2010.

[26] P. Dong, Y.-K. Chen, G.-H. Duan, and D. T. Neilson, "Silicon photonic devices and integrated circuits," Nanophotonics, vol. 3, no 4-5, pp. 215-228, 2014.

[27] H. Lin, Z. Luo, T. Gu, et al., "Mid-infrared integrated photonics on silicon: a perspective," Nanophotonics, vol. 7, no. 2, pp. 393-420, 2017.

[28] T. Rudolph, "Why I am optimistic about the silicon-photonic route to quantum computing," APL Photonics, vol. 2, no. 3, p. $030901,2017$.

[29] Y. Shen, N. C. Harris, S. Skirlo, et al., "Deep learning with coherent nanophotonic circuits," Nat. Photonics, vol. 11, no. 7, p. 441, 2017.

[30] C. E. Shannon, “Communication in the presence of noise," Proc. IRE, vol. 37, no. 1, 1949, pp. 10-21. 
[31] E. Ip, A. P. T. Lau, D. J. Barros, and J. M. Kahn, "Coherent detection in optical fiber systems," Opt. Express, vol. 16, no. 2, pp. 753-791, 2008.

[32] P. J. Winzer, "High-spectral-efficiency optical modulation formats," J. Lightwave Technol., vol. 30, no. 24, pp. 3824-3835, 2012.

[33] G. Bosco, V. Curri, A. Carena, P. Poggiolini, and F. Forghieri, “On the performance of Nyquist-WDM terabit superchannels based on PM-BPSK, PM-QPSK, PM-8QAM or PM-16QAM subcarriers," J. Lightwave Technol, vol. 29, no. 1, pp. 53-61, 2010.

[34] R.-J. Essiambre and R. W. Tkach, "Capacity trends and limits of optical communication networks," Proc. IEEE, vol. 100, no. 5, 2012, pp. 1035-1055.

[35] J. Lin, H. Sepehrian, L. Rusch, and W. Shi, "Single-carrier 720 Gb/ S DP-32QAM transmission using a silicon photonic IQ modulator," Optica, vol. 27, no. 4, pp. 5610-5619, 2018.

[36] J.-X. Cai, M. V. Mazurczyk, H. Batshon, et al., "Performance comparison of probabilistically shaped qam formats and hybrid shaped apsk formats with coded modulation," J. Lightwave Technol., vol. 38, no. 12, pp. 3280-3288, 2020.

[37] A. Pilipetskii and G. Mohs, "Technology evolution and capacity growth in undersea cables," in Optical Fiber Communication Conference, San Diego, IEEE, 2020, pp. W4E-2.

[38] J. Renaudier, A. C. Meseguer, A. Ghazisaeidi, et al., "First 100-nm continuous-band WDM transmission system with $115 \mathrm{~Tb} / \mathrm{s}$ transport over $100 \mathrm{~km}$ using novel ultra-wideband semiconductor optical amplifiers," in 2017 European Conference on Optical Communication (ECOC), Gothenburg, IEEE, 2017, pp. 1-3. https://doi.org/10.1109/ECOC.2017.8346084.

[39] A. Arnould, A. Ghazisaeidi, D. Le Gac, et al., "103 nm ultrawideband hybrid Raman/SOA transmission over $3 \times 100 \mathrm{~km}$ SSMF," J. Lightwave Technol., vol. 38, no. 2, pp. 504-508, 2020.

[40] M. Ionescu, D. Lavery, A. Edwards, et al., “74.38 Tb/s transmission over $6300 \mathrm{~km}$ single mode fibre enabled by $\mathrm{C}+\mathrm{L}$ amplification and geometrically shaped PDM-64QAM," J. Lightwave Technol., vol. 38, no. 2, pp. 531-537, 2020.

[41] T. Baehr-Jones, T. Pinguet, P. Lo Guo-Qiang, S. Danziger, D. Prather, and $M$. Hochberg, "Myths and rumours of silicon photonics," Nat. Photonics, vol. 6, no. 4, pp. 206-208, 2012.

[42] T.-J. Lu, M. Fanto, H. Choi, et al., "Aluminum nitride integrated photonics platform for the ultraviolet to visible spectrum," Opt. Express, vol. 26, no. 9, Apr 2018, p. 11147. [Online]. Available at: https://www.osapublishing.org/abstract.cfm?URI=0e-26-9-11147.

[43] J. Chiles and S. Fathpour, "Silicon photonics beyond silicon-oninsulator,"J. Opt., vol. 19, no. 5, 2017, https://doi.org/10.1088/ 2040-8986/aa5f5e.

[44] T. Hu, B. Dong, X. Luo, et al., "Silicon photonic platforms for midinfrared applications [Invited]," Photonics Res., vol. 5, no. 5, p. 417, 2017.

[45] D. X. Xu, J. H. Schmid, G. T. Reed, et al., "Silicon photonic integration platform-Have we found the sweet spot?," IEEEJ. Sel. Top. Quant. Electron., vol. 20, no. 4, 2014. https://doi.org/2014, 10.1109/JSTQE.2014.2299634.

[46] M. Hochberg, N. Harris, R. Ding, et al., "Silicon photonics: the next fabless semiconductor industry," IEEE Solid-State Circ. Mag., vol. 5, no. 1, pp. 48-58, 2013

[47] D. Thomson, A. Zilkie, J. E. Bowers, et al., "Roadmap on silicon photonics,” J. Opt., vol. 18, no. 7, pp. 1-20, 2016 [Online]. Available at:
[48] R. Marchetti, C. Lacava, L. Carroll, K. Gradkowski, and P. Minzioni, "Coupling strategies for silicon photonics integrated chips," Photonics Res., vol. 7, no. 2, Feb 2019, pp. 201-239 [Online]. Available at: http://www.osapublishing. org/prj/abstract.cfm?URI=pri-7-2-201.

[49] D. Vermeulen and C. V. Poulton, "Optical interfaces for silicon photonic circuits,” Proc. IEEE, vol. 106, no. 12, 2018, pp. 2270-2280.

[50] J. Sun, E. Timurdogan, A. Yaacobi, E. S. Hosseini, and M. R. Watts, "Large-scale nanophotonic phased array," Nature, vol. 493, no. 7431, pp. 195-199, 2013.

[51] L. Jia, C. Li, T.-Y. Liow, and G.-Q. Lo, “Efficient suspended coupler with loss less than- $1.4 \mathrm{~dB}$ between si-photonic waveguide and cleaved single mode fiber," J. Lightwave Technol., vol. 36, no. 2, pp. 239-244, 2018.

[52] Y. Painchaud, M.-j. Picard, C. Latrasse, and M. Poulin, "Spot-size converter for optical mode conversion and coupling between two waveguides," July 11 2017, US Patent 9,703,047.

[53] R. Halir, A. Ortega-Moñux, D. Benedikovic, et al., "Subwavelength-grating metamaterial structures for silicon photonic devices," Proc. IEEE, vol. 106, no. 12, 2018, pp. 2144-2157.

[54] S. Bhat, M. Harjanne, F. Sun, et al., "Low loss devices fabricated on the open Access $3 \mu \mathrm{m}$ SOI waveguide platform at VTT," in European Conference on Integrated Optics, Ghent, 2019.

[55] A. Rahim, E. Ryckeboer, A. Z. Subramanian, et al., "Expanding the silicon photonics portfolio with silicon nitride photonic integrated circuits," J. Lightwave Technol., vol. 35, no. 4, pp. 639-649, 2017.

[56] P. Muñoz, G. Micó, L. A. Bru, et al., "Silicon nitride photonic integration platforms for visible, near-infrared and mid-infrared applications," Sensors, vol. 17, no. 9, pp. 1-25, 2017.

[57] Y. Huang, J. Song, X. Luo, T.-Y. Liow, and G.-Q. Lo, "CMOS compatible monolithic multi-layer $\mathrm{Si}_{3} \mathrm{~N}_{4}$-on-SOI platform for lowloss high performance silicon photonics dense integration," Opt. Express, vol. 22, no. 18, p. 21859, 2014.

[58] W. D. Sacher, J. C. Mikkelsen, Y. Huang, et al., "Monolithically integrated multilayer silicon nitride-on-silicon waveguide platforms for 3-D photonic circuits and devices," Proc. IEEE, vol. 106, no. 12, 2018, pp. 2232-2245.

[59] S. Guerber, C. Alonso-Ramos, D. Benedikovic, et al., "Integrated SiN on SOI dual photonic devices for advanced datacom solutions," in Silicon Photonics: From Fundamental Research to Manufacturing, Strasbourg, Proc. SPIE, vol. 10686, 2018. https://doi.org/10.1117/12.2306160.

[60] E. Durán-Valdeiglesias, S. Guerber, D. Oser, et al., "Dualpolarization silicon nitride Bragg filters with low thermal sensitivity," Opt. Lett., vol. 44, no. 18, p. 4578, 2019.

[61] Q. Han, J. St-Yves, Y. Chen, M. Ménard, and W. Shi, "Polarizationinsensitive silicon nitride arrayed waveguide grating," Opt. Lett., vol. 44, no. 16, pp. 3976-3979, 2019.

[62] A. L. Gaeta, M. Lipson, and T. J. Kippenberg, "Photonic-chipbased frequency combs," Nat. Photonics, vol. 13, no. 3, pp. 158-169, 2019.

[63] T. Hiramoto, "Five nanometre CMOS technology," Nat. Electron., vol. 2, no. 12, pp. 557-558, 2019 [Online]. Available at:.

[64] J. S. Orcutt, A. Khilo, C. W. Holzwarth, et al., "Nanophotonic integration in state-of-the-art CMOS foundries," Opt. Express, vol. 19 , no. 3 , p. 2335,2011 
[65] Z. Yong, S. Shopov, J. C. Mikkelsen, et al., "Flip-chip integrated silicon Mach-Zehnder modulator with a $28 \mathrm{~nm}$ fully depleted silicon-on-insulator CMOS driver," Opt. Express, vol. 25, no. 6, p. 6112, 2017.

[66] M. Cignoli, G. Minoia, M. Repossi, et al., “A 1310nm 3Dintegrated silicon photonics Mach-Zehnder-based transmitter with $275 \mathrm{~mW}$ multistage CMOS driver achieving $6 \mathrm{~dB}$ extinction ratio at $25 \mathrm{~Gb} / \mathrm{s}$," in 2015 IEEE International Solid-State Circuits Conference, San Francisco, CA, IEEE, 2015, pp. 1-3. https://doi. org/10.1109/ISSCC.2015.7063103.

[67] H. D. Thacker, Y. Luo, J. Shi, et al., "Flip-chip integrated silicon photonic bridge chips for sub-picojoule per bit optical links," in 2010 Proceedings 60th Electronic Components and Technology Conference (ECTC), pp. 240-246, IEEE, Las Vegas, 2010. https:// doi.org/10.1109/ECTC.2010.5490965.

[68] K. T. Settaluri, S. Lin, S. Moazeni, et al., "Demonstration of an optical chip-to-chip link in a 3D integrated electronic-photonic platform," in European Solid-State Circuits Conference, vol. 2015-October, 2015, pp. 156-159.

[69] F. Boeuf, S. Cremer, E. Temporiti, et al., "Recent progress in Silicon Photonics R\&D and manufacturing on $300 \mathrm{~mm}$ wafer platform," in Conference on Optical Fiber Communication, Technical Digest Series, vol. 2015-June, pp. 7-9, 2015.

[70] Mounier Eric, Debray Alexis, Malinge Jean-Louis. Yole Developpement: Silicon Photonics 2020 - Market \& Technology Report. Technical Report 2020.

[71] L. Chrostowski, H. Shoman, M. Hammood, et al., "Silicon photonic circuit design using rapid prototyping foundry process design kits," IEEE J. Sel. Top. Quant. Electron., vol. 25, no. 5, pp. 1-26, 2019.

[72] R. Going, M. Lauermann, R. Maher, et al., “Multi-channel inp-based coherent pics with hybrid integrated sige electronics operating up to $100 \mathrm{GBd}$, 32QAM," in 2017 European Conference on Optical Communication (ECOC), Gothenburg, IEEE, 2017, pp. 1-3. https://doi.org/10.1109/ECOC.2017.8346096.

[73] Y. Ogiso, J. Ozaki, Y. Ueda, et al., "Over $67 \mathrm{GHz}$ bandwidth and $1.5 \mathrm{~V} \mathrm{~V}_{\pi}$ InP-based optical IQ modulator with nipn heterostructure," J. Lightwave Technol., vol. 35, no. 8, pp. 1450-1455, 2017.

[74] R. Going, S. Wolf, R. Maher, et al., "Inp-based coherent PICs for 100 Gbaud operation," in Signal Processing in Photonic Communications, Burlingame: Optical Society of America, 2019, pp. SpM4E-3. https://doi.org/10.1364/SPPCOM.2019.SpM4E.3.

[75] V. Lal, P. Studenkov, T. Frost, et al., "1.6 Tbps coherent 2-channel transceiver using a monolithic Tx/Rx InP PIC and single SiGe ASIC," in Optical Fiber Communication Conference, San Diego: Optical Society of America, 2020, pp. M3A-2. https://doi.org/ 10.1364/OFC.2020.M3A.2.

[76] T. Pinguet, S. Denton, S. Gloeckner, et al., "High-volume manufacturing platform for silicon photonics," Proc. IEEE, vol. 106, no. 12 , 2018, pp. 2281-2290.

[77] G. Li, "Recent advances in coherent optical communication," Adv. Opt. Photonics, vol. 1, no. 2, pp. 279-307, 2009.

[78] 2020 [Online]. Available at: http://ir.acacia-inc.com/newsreleases/news-release-details/first-400g-transmission-across$6600 \mathrm{~km}$-trans-atlantic-marea-cable.

[79] A. Novack, M. Streshinsky, T. Huynh, et al., "A silicon photonic transceiver and hybrid tunable laser for 64 Gbaud coherent communication," In Optical Fiber Communication Conference,
San Diego: Optical Society of America, 2018, pp. Th4D-4. https://doi.org/10.1364/OFC.2018.Th4D.4.

[80] S. Yamanaka, Y. Ikuma, T. Itoh, et al., "Silicon photonics coherent optical subassembly with EO and OE bandwidths of over $50 \mathrm{GHz}$," in Optical Fiber Communication Conference, San Diego: Optical Society of America, 2020, pp. Th4A-4. https:// doi.org/10.1364/OFC.2020.Th4A.4.

[81] C. Doerr, L. Chen, T. Nielsen, et al., "OESC,,,, and Lb and silicon photonics coherent modulator/receiver," in 2016 Optical Fiber Communications Conference and Exhibition (OFC), Anaheim: IEEE, 2016, pp. 1-3.

[82] C. Doerr and L. Chen, "Silicon photonics in optical coherent systems," Proc. IEEE, vol. 106, no. 12, Dec 2018, pp. 2291-2301.

[83] J. Michel, J. Liu, and L. C. Kimerling, "High-performance Ge-on-Si photodetectors," Nat. Photonics, vol. 4, no. 8, p. 527, 2010.

[84] M. Piels and J. E. Bowers, "Photodetectors for silicon photonic integrated circuits," Photodetectors, Woodhead Publishing, 2016, pp. 3-20. https://doi.org/10.1016/B978-1-78242-445-1. 00001-4.

[85] Y. Zhang, S. Yang, Y. Yang, et al., "A high-responsivity photodetector absent metal-germanium direct contact," Opt. Express, vol. 22, no. 9, pp. 11 367-11 375, 2014.

[86] D. Feng, S. Liao, P. Dong, et al., “High-speed Ge photodetector monolithically integrated with large cross-section silicon-oninsulator waveguide," Appl. Phys. Lett., vol. 95, no. 26, p. 261105, 2009.

[87] H. Chen, M. Galili, P. Verheyen, et al., "100-Gbps RZ data reception in 67-GHz Si-contacted germanium waveguide pin photodetectors," J. Lightwave Technol., vol. 35, no. 4, pp. 722-726, 2016.

[88] S. M. Sze and K. K. Ng, Physics of Semiconductor Devices, John Wiley \& Sons, 2006.

[89] B. Yang, J.-Y. J. Lin, S. Gupta, et al., “Low-contact-resistivity nickel germanide contacts on $\mathrm{n}+\mathrm{Ge}$ with phosphorus/antimony co-doping and Schottky barrier height lowering," in 2012 International Silicon-Germanium Technology and Device Meeting (ISTDM), Berkeley: IEEE, 2012, pp. 1-2. https://doi.org/ 10.1109/ISTDM.2012.6222472.

[90] A. Uetake, N. Yasuoka, T. Kurahashi, et al., "Wide-input-power dynamic range, 40-GHz waveguide pin germanium photodetector for photonic integrated circuit," in 2018 European Conference on Optical Communication (ECOC), Rome: IEEE, 2018, pp. 1-3. https://doi.org/10.1109/ECOC.2018.8535465.

[91] H. T. Chen, P. Verheyen, P. De Heyn, et al., “High-responsivity low-voltage $28-\mathrm{Gb} / \mathrm{s}$ Ge pin photodetector with silicon contacts," J. Lightwave Technol., vol. 33, no. 4, pp. 820-824, 2014.

[92] Y. Kang, H.-D. Liu, M. Morse, et al., “Monolithic germanium/silicon avalanche photodiodes with $340 \mathrm{GHz}$ gainbandwidth product," Nat. Photonics, vol. 3, no. 1, pp. 59-63, 2009.

[93] Z. Huang, C. Li, D. Liang, et al., " $25 \mathrm{Gbps}$ low-voltage waveguide Si-Ge avalanche photodiode," Optica, vol. 3, no. 8, pp. 793-798, 2016.

[94] N. J. Martinez, C. T. Derose, R. W. Brock, et al., "High performance waveguide-coupled Ge-on-Si linear mode avalanche photodiodes," Opt. Express, vol. 24, no. 17, pp. 19 072-19 081, 2016.

[95] J. C. Campbell, "Recent advances in avalanche photodiodes," J. Lightwave Technol., vol. 34, no. 2, pp. 278-285, 2016. 
[96] J. Zhang, B. P.-P. Kuo, and S. Radic, “64Gb/s PAM4 and 160Gb/ s 16QAM modulation reception using a low-voltage Si-Ge waveguide-integrated APD," Opt. Express, vol. 28, no. 16, pp. $23266-23273,2020$.

[97] B. Wang, Z. Huang, W. V. Sorin, et al., “A low-voltage Si-Ge avalanche photodiode for high-speed and energy efficient silicon photonic links," J. Lightwave Technol., vol. 38, no. 12, pp. 3156-3163, 2020.

[98] K. Voigt, L. Zimmermann, G. Winzer, H. Tian, B. Tillack, and K. Petermann, "C-band optical $90^{\circ}$ hybrids in silicon nanowaveguide technology," IEEE Photonics Technol. Lett., vol. 23, no. 23, pp. 1769-1771, 2011.

[99] R. Halir, G. Roelkens, A. Ortega-Moñux, and J. WangüemertPérez, "High-performance 90 hybrid based on a silicon-oninsulator multimode interference coupler," Opt. Lett., vol. 36, no. 2, pp. 178-180, 2011.

[100] M. Yin, W. Yang, Y. Huang, et al., "Compact and wideband optical $90^{\circ}$ hybrid based on silicon-on-insulator," in 10th International Conference on Group IV Photonics, Seoul: IEEE, 2013, pp. 57-58. https://doi.org/10.1109/Group4.2013. 6644480.

[101] W. Yang, M. Yin, Y. Li, X. Wang, and Z. Wang, "Ultra-compact optical $90^{\circ}$ hybrid based on a wedge-shaped $2 \times 4 \mathrm{MMI}$ coupler and a $2 \times 2 \mathrm{MMI}$ coupler in silicon-on-insulator," Opt. Express, vol. 21, no. 23, pp. 28 423-28 431, 2013.

[102] S. Faralli, G. Meloni, F. Gambini, J. Klamkin, L. Potì, and G. Contestabile, "A compact silicon coherent receiver without waveguide crossing," IEEE Photonics J., vol. 7, no. 4, pp. 1-6, 2015.

[103] H. Guan, Y. Ma, R. Shi, et al., "Compact and low loss $90^{\circ}$ optical hybrid on a silicon-on-insulator platform," Opt. Express, vol. 25, no. 23, pp. 28 957-28 968, 2017.

[104] A. Melikyan and P. Dong, "Adiabatic mode converters for silicon photonics: power and polarization broadband manipulators," APL Photonics, vol. 4, no. 3, p. 030803, 2019.

[105] S.-H. Jeong and K. Morito, "Optical $90^{\circ}$ hybrid with broad operating bandwidth of 94 nm," Opt. Lett., vol. 34, no. 22, pp. 3505-3507, 2009.

[106] R. Soref and B. Bennett, "Electrooptical effects in silicon," IEEE J. Quant. Electron, vol. 23, no. 1, pp. 123-129, 1987.

[107] W. Shi, Y. Xu, H. Sepehrian, S. LaRochelle, and L. A. Rusch, "Silicon photonic modulators for PAM transmissions," J. Opt., vol. 20, no. 8, p. 083002, 2018.

[108] J. Witzens, “High-speed silicon photonics modulators," Proc. IEEE, vol. 106, no. 12, Dec 2018, pp. 2158-2182.

[109] A. D. Bristow, N. Rotenberg, and H. M. Van Driel, "Two-photon absorption and Kerr coefficients of silicon for 850-2200 nm," Appl. Phys. Lett., vol. 90, no. 19, p. 191104, 2007.

[110] H. Sepehrian, A. Yekani, W. Shi, and L. A. Rusch, “Assessing performance of silicon photonic modulators for pulse amplitude modulation," IEEE J. Sel. Top. Quant. Electron., vol. 24, no. 6, pp. 1-10, 2018.

[111] H. Sepehrian, J. Lin, L. A. Rusch, and W. Shi, "Silicon photonic IQ modulators for $400 \mathrm{~Gb} / \mathrm{s}$ and beyond," J. Lightwave Technol., vol. 37, no. 13, pp. 3078-3086, 2019.

[112] S. Zhalehpour, M. Guo, J. Lin, et al., "All silicon IQ modulator with $1 \mathrm{~Tb} / \mathrm{s}$ line rate," in Optical Fiber Communication Conference, San Diego: Optical Society of America, 2020, p. W3D.6. https://doi.org/10.1364/OFC.2020.W3D.6.
[113] J. Zhou, J. Wang, and Q. Zhang, "Silicon photonics for 100Gbaud," in Optical Fiber Communication Conference, San Diego: Optical Society of America, 2020, p. T3H.4. https://doi. org/10.1364/OFC.2020.T3H.4.

[114] S. Zhalehpour, M. Guo, J. Lin, et al., "System optimization of an all-silicon IQ modulator: Achieving 100-Gbaud dualpolarization 32QAM," J. Lightwave Technol., vol. 38, no. 2, pp. 256-264, Jan 2020.

[115] S. Zhalehpour, J. Lin, W. Shi, and L. A. Rusch, "Reduced-size lookup tables enabling higher-order QAM with all-silicon IQ modulators," Opt. Express, vol. 27, no. 17, pp. 24 243-24 259, 2019.

[116] B. Milivojevic, S. Wiese, S. Anderson, T. Brenner, M. Webster, and B. Dama, "Demonstration of optical transmission at bit rates of up to $321.4 \mathrm{~Gb} / \mathrm{s}$ using compact silicon based modulator and linear BiCMOS MZM driver," J. Lightwave Technol., vol. 35, no. 4, pp. 768-774, Feb 2017.

[117] S. Wolf, H. Zwickel, C. Kieninger, et al., "Coherent modulation up to $100 \mathrm{GBd}$ 16QAM using silicon-organic hybrid (soh) devices," Opt. Express, vol. 26, no. 1, pp. 220-232, 2018.

[118] W. Heni, Y. Fedoryshyn, B. Baeuerle, et al., "Plasmonic IQ modulators with attojoule per bit electrical energy consumption," Nat. Commun., vol. 10, no. 1, pp. 1-8, 2019.

[119] H. Bahrami, H. Sepehrian, C. S. Park, L. A. Rusch, and W. Shi, "Time-domain large-signal modeling of traveling-wave modulators on SOI," J. Lightwave Technol., vol. 34, no. 11, pp. 2812-2823, 2016.

[120] R. Dubé-Demers, J. St-Yves, A. Bois, et al., “Analytical modeling of silicon microring and microdisk modulators with electrical and optical dynamics," J. Lightwave Technol., vol. 33, no. 20, pp. 4240-4252, 2015.

[121] S. Zhalehpour, J. Lin, H. Sepehrian, W. Shi, and L. A. Rusch, "Mitigating pattern dependent nonlinearity in SiP IQ-modulators via iterative learning control predistortion," Opt. Express, vol. 26, no. 21, Oct 2018, pp. 27 639-27 649 [Online]. Available at: http://www.opticsexpress.org/abstract.cfm? URI=oe-26-21-27639.

[122] L. Vivien, A. Polzer, D. Marris-Morini, et al., "Zero-bias 40Gbit/s germanium waveguide photodetector on silicon," Opt. Express, vol. 20, no. 2, Jan 2012, pp. 1096-1101 [Online]. Available at: http://www.opticsexpress.org/abstract.cfm?URI=0e-20-21096.

[123] A. Novack, M. Gould, Y. Yang, et al., “Germanium photodetector with $60 \mathrm{GHz}$ bandwidth using inductive gain peaking," Opt. Express, vol. 21, no. 23, pp. 28387-28393, 2013.

[124] J. Sun, R. Kumar, M. Sakib, J. B. Driscoll, H. Jayatilleka, and H. Rong, "A $128 \mathrm{~Gb} / \mathrm{s}$ PAM4 silicon microring modulator with integrated thermo-optic resonance tuning," J. Lightwave Technol., vol. 37, no. 1, pp. 110-115, 2019.

[125] D. Patel, S. Ghosh, M. Chagnon, et al., "Design, analysis, and transmission system performance of a $41 \mathrm{GHz}$ silicon photonic modulator," Opt. Express, vol. 23, no. 11, pp. 14263-14287, 2015.

[126] M. Li, L. Wang, X. Li, X. Xiao, and S. Yu, "Silicon intensity MachZehnder modulator for single lane $100 \mathrm{~Gb} / \mathrm{s}$ applications," Photonics Res., vol. 6, no. 2, pp. 109-116, 2018.

[127] A. Simard, B. Filion, D. Patel, D. Plant, and S. LaRochelle, "Segmented silicon MZM for PAM-8 transmissions at $114 \mathrm{~Gb} / \mathrm{s}$ 
with binary signaling," Opt. Express, vol. 24, no. 17, pp. 19467-19472, 2016.

[128] J. Lin, H. Sepehrian, L. A. Rusch, and W. Shi, "Joint digital and optical pre-compensation for $720 \mathrm{gb} / \mathrm{s}$ all-silicon IQ modulator single carrier transmission," in 2018 European Conference on Optical Communication (ECOC), Rome: IEEE, 2018, pp. 1-3. https://doi.org/10.1109/ECOC.2018.8535504.

[129] P. Dong, C. Xie, L. L. Buhl, Y.-K. Chen, J. H. Sinsky, and G. Raybon, "Silicon in-phase/quadrature modulator with onchip optical equalizer," J. Lightwave Technol., vol. 33, no. 6, pp. 1191-1196, 2015.

[130] C. R. Doerr, "Silicon-photonics-enabled low-cost and smallfootprint fiber-optic communications (Conference Presentation)," in Integrated Optics: Devices, Materials, and Technologies XXIV, vol. 11283, S. M. GarcÃa-Blanco and P. Cheben, International Society for Optics and Photonics. SPIE, 2020 [Online]. Available at: https://doi.org/10.1117/12. 2554478.

[131] N. Sambo, P. Castoldi, A. D’Errico, et al., "Next generation sliceable bandwidth variable transponders," IEEE Commun. Mag., vol. 53, no. 2, pp. 163-171, 2015.

[132] V. Lal, J. Summers, N. Kim, et al., "Extended C-band tunable multi-channel InP-based coherent transmitter PICs," J. Lightwave Technol., vol. 35, no. 7, pp. 1320-1327, 2017.

[133] M. Qiu, Q. Zhuge, X. Xu, M. Chagnon, M. Morsy-Osman, and D. V. Plant, "Subcarrier multiplexing using dacs for fiber nonlinearity mitigation in coherent optical communication systems," in Optical Fiber Communication Conference, San Francisco: Optical Society of America, 2014, pp. Tu3J-2. https://doi.org/10.1364/OFC.2014.M2A.5.

[134] J. Lin, H. Sepehrian, Y. Xu, L. A. Rusch, and W. Shi, “Optical frequency comb generation using a CMOS compatible silicon dual-drive MZM," IEEE Photonics Technol. Lett, vol. 30, no. 7, pp. 1495-1498, 2018.

[135] P. Abolghasem, L. Dardis, A. Diba, et al., "Monolithically integrated l-band pics and transceiver modules with $6 \lambda \times 200$ Gbps (1.2 Tbps) for C+ L band communication systems," Opt. Express, vol. 27, no. 12, pp. 16483-16492, 2019.

[136] P. S. Khodashenas, J. M. Rivas-Moscoso, D. Siracusa, et al., "Comparison of spectral and spatial super-channel allocation schemes for SDM networks," J. Lightwave Technol., vol. 34, no. 11, pp. 2710-2716, 2016.

[137] R. Soref, "Mid-infrared photonics in silicon and germanium," Nat. Photonics, vol. 4, no. 8, pp. 495-497, 2010 [Online]. Available at:.

[138] Z. Li, A. Heidt, N. Simakov, et al., "Diode-pumped wideband thulium-doped fiber amplifiers for optical communications in the 1800-2050 nm window," Opt. Express, vol. 21, no. 22, pp. 26 450-26 455, 2013.

[139] Y. Jung, P. C. Shardlow, M. Belal, et al., "First demonstration of a $2 \mu \mathrm{m}$ few-mode TDFA for mode division multiplexing," Opt. Express, vol. 22, no. 9, May 2014, pp. 10544-10549.

[140] D. Richardson, N. Wheeler, Y. Chen, et al., "Hollow core fibres and their applications," in Optical Fiber Communication Conference, Los Angeles: Optical Society of America, 2017, pp. Tu3H-1. https://doi.org/10.1364/OFC.2017.Tu3H.1.

[141] Z. Liu, Y. Chen, Z. Li, et al., "High-capacity directly modulated optical transmitter for $2-\mu \mathrm{m}$ spectral region," J. Lightwave Technol., vol. 33, no. 7, pp. 1373-1379, 2015.
[142] D. Kong, Y. Liu, Z. Ren, et al., "Generation and coherent detection of $2-\mu \mathrm{m}$-band WDM-QPSK signals by on-chip spectral translation," in Optical Fiber Communication Conference, San Diego: Optical Society of America, 2020, pp. M1l-4. https:// doi.org/10.1364/OFC.2020.M11.4.

[143] M. Nedeljkovic, R. Soref, and G. Z. Mashanovich, "Free-carrier electrorefraction and electroabsorption modulation predictions for silicon over the 1-14- $\mu \mathrm{m}$ infrared wavelength range," IEEE Photonics J., vol. 3, no. 6, pp. 1171-1180, 2011.

[144] W. Cao, D. Hagan, D. J. Thomson, et al., "High-speed silicon modulators for the $2 \mu \mathrm{m}$ wavelength band," Optica, vol. 5, no. 9, pp. 1055-1062, 2018.

[145] W. Li, M. Li, H. Zhang, et al., " $50 \mathrm{Gbit} / \mathrm{s}$ silicon modulator operated at $1950 \mathrm{~nm}$," in Optical Fiber Communication Conference, San Diego: Optical Society of America, 2020, p. M1D.4. https://doi.org/10.1364/OFC.2020.M1D.4.

[146] Z. Sheng, L. Liu, J. Brouckaert, S. He, and D. Van Thourhout, "InGaAs PIN photodetectors integrated on silicon-on-insulator waveguides," Opt. Express, vol. 18, no. 2, pp. 1756-1761, 2010.

[147] O. I. Dosunmu, D. D. Cannon, M. K. Emsley, L. C. Kimerling, and M. S. Unlu, "High-speed resonant cavity enhanced ge photodetectors on reflecting si substrates for 1550-nm operation," IEEE Photonics Technol. Lett., vol. 17, no. 1, pp. 175-177, 2004.

[148] Z. Su, E. S. Hosseini, E. Timurdogan, et al., "Whispering gallery germanium-on-silicon photodetector," Opt. Lett., vol. 42, no. 15, Aug 2017, pp. 2878-2881 [Online]. Available at: http://ol. osa.org/abstract.cfm?URI=ol-42-15-2878.

[149] H. Tran, T. Pham, J. Margetis, et al., "Si-based GeSn photodetectors toward mid-infrared imaging applications," ACS Photonics, vol. 6, no. 11, pp. 2807-2815, 2019.

[150] Y. Dong, W. Wang, S. Xu, et al., "Two-micron-wavelength germanium-tin photodiodes with low dark current and gigahertz bandwidth," Opt. Express, vol. 25, no. 14, pp. 15818-15827, 2017.

[151] J. J. Ackert, D. J. Thomson, L. Shen, et al., "High-speed detection at two micrometres with monolithic silicon photodiodes," Nat. Photonics, vol. 9, no. 6, p. 393, 2015.

[152] R. Anthony, D. E. Hagan, D. Genuth-Okon, et al., "Extended wavelength responsivity of a germanium photodetector integrated with a silicon waveguide exploiting the indirect transition," IEEE J. Sel. Top. Quant. Electron., vol. 26, no. 2, pp. 1-7, 2019.

[153] S. Fathololoumi, K. Nguyen, H. Mahalingam, et al., "1.6 Tbps silicon photonics integrated circuit for co-packaged optical-IO switch applications," in Optical Fiber Communication Conference, San Diego: Optical Society of America, 2020, pp. T3H-1. https://doi.org/10.1364/OFC.2020.T3H.1.

[154] B. Buscaino, B. D. Taylor, and J. M. Kahn, "Multi-Tb/s-per-fiber coherent co-packaged optical interfaces for data center switches," J. Lightwave Technol., vol. 37, no. 13, pp. 3401-3412, 2019.

[155] L. Jiang, X. Chen, K. Kim, G. de Valicourt, Z. R. Huang, and P. Dong, "Electro-optic crosstalk in parallel silicon photonic Mach-Zehnder modulators," J. Lightwave Technol., vol. 36, no. 9, pp. 1713-1720, 2018.

[156] W. Yao, G. Gilardi, N. Calabretta, M. K. Smit, and M. J. Wale, "Experimental and numerical study of electrical crosstalk in photonic-integrated circuits," J. Lightwave Technol., vol. 33, no. 4, pp. 934-942, 2015. 
[157] X. Chen, P. Dong, S. Chandrasekhar, et al., “Characterization and digital pre-compensation of electro-optic crosstalk in silicon photonics I/Q modulators," in 42nd European Conference on Optical Communication, Dusseldorf: VDE, 2016, pp. 1-3.

[158] Q. Xu, B. Schmidt, S. Pradhan, and M. Lipson, "Micrometrescale silicon electro-optic modulator," Nature, vol. 435, no. 7040, pp. 325-327, 2005.

[159] E. Timurdogan, C. M. Sorace-Agaskar, J. Sun, E. S. Hosseini, A. Biberman, and M. R. Watts, "An ultralow power athermal silicon modulator," Nat. Commun., vol. 5, no. 1, pp. 1-11, 2014.

[160] R. Dubé-Demers, S. LaRochelle, and W. Shi, “Ultrafast pulseamplitude modulation with a femtojoule silicon photonic modulator," Optica, vol. 3, no. 6, Jun 2016, pp. 622-627 [Online]. Available at: http://www.osapublishing.org/optica/ abstract.cfm?URI=optica-3-6-622.

[161] M. Rakowski, Y. Ban, P. De Heyn, et al., “Hybrid 14nm FinFET silicon photonics technology for low-power $\mathrm{Tb} / \mathrm{s} / \mathrm{mm} 2$ optical I/O," in 2018 IEEE Symposium on VLSI Technology, Honolulu: IEEE, 2018, pp. 221-222. https://doi.org/10.1109/VLSIT.2018. 8510668 .

[162] A. Moscoso-Mártir, A. Tabatabaei-Mashayekh, J. Müller, et al., "8-channel WDM silicon photonics transceiver with SOA and semiconductor mode-locked laser," Opt. Express, vol. 26, no. 19, pp. 25 446-25 459, 2018.

[163] Y. Xu, J. Lin, R. Dubé-Demers, S. LaRochelle, L. Rusch, and W. Shi, "Integrated flexible-grid wdm transmitter using an optical frequency comb in microring modulators," Opt. Lett., vol. 43, no. 7, pp. 1554-1557, 2018.

[164] P. Dong, C. Xie, L. L. Buhl, and Y.-K. Chen, "Silicon microring modulators for advanced modulation formats," in Optical Fiber Communication Conference, Anaheim: Optical Society of America, pp. OW4J.2, 2013. https://doi.org/10.1364/OFC.2013. OW4J.2.

[165] C.-M. Chang, P. Dong, C. Gui, and G. de Valicourt, "Low-chirp push-pull microring modulators," in Optical Fiber Communication Conference, Anaheim: Optical Society of America, p. Th4H.2., 2016. https://doi.org/10.1364/OFC.2016. Th4H.2.

[166] R. Li, D. Patel, E. El-Fiky, et al., "High-speed low-chirp PAM-4 transmission based on push-pull silicon photonic microring modulators," Opt. Express, vol. 25, no. 12, pp. 13222-13229, Jun 2017 [Online]. Available at: http://www.opticsexpress.org/ abstract.cfm?URI=0e-25-12-13222.

[167] H. Li, G. Balamurugan, M. Sakib, et al., "A 112 Gb/s PAM4 silicon photonics transmitter with microring modulator and CMOS driver," J. Lightwave Technol., vol. 38, no. 1, pp. 131-138, 2020

[168] M. J. Shin, Y. Ban, B.-M. Yu, J. Rhim, L. Zimmermann, and W.Y. Choi, "Parametric characterization of self-heating in depletion-type si micro-ring modulators," IEEE J. Sel. Top. Quant. Electron., vol. 22, no. 6, pp. 116-122, 2016.

[169] M. de Cea, A. H. Atabaki, and R. J. Ram, "Power handling of silicon microring modulators,” Opt. Express, vol. 27, no. 17, pp. 24274-24285, 2019.

[170] T. Baba, "Slow light in photonic crystals," Nat. Photonics, vol. 2, no. 8, p. 465, 2008.

[171] Y. Terada, K. Kondo, R. Abe, and T. Baba, "Full C-band si photonic crystal waveguide modulator," Opt. Lett., vol. 42, no. 24, pp. 5110-5112, 2017
[172] S. Romero-García, A. Moscoso-Mártir, S. S. Azadeh, et al., "High-speed resonantly enhanced silicon photonics modulator with a large operating temperature range," Opt. Lett., vol. 42, no. 1, pp. 81-84, 2017.

[173] O. Jafari, W. Shi, and S. Larochelle, "Mach-Zehnder silicon photonic modulator assisted by phase-shifted Bragg gratings," IEEE Photonics Technol. Lett., vol. 32, no. 8, pp. 445-448, 2020.

[174] K. Hojo, Y. Terada, N. Yazawa, T. Watanabe, and T. Baba, "Compact QPSK and PAM modulators with si photonic crysta slow-light phase shifters," IEEE Photonics Technol. Lett., vol. 28, no. 13, pp. 1438-1441, 2016.

[175] X. Wang, W. Shi, H. Yun, S. Grist, N. A. F. Jaeger, and L. Chrostowski, "Narrow-band waveguide Bragg gratings on SOI wafers with CMOS-compatible fabrication process," Opt. Express, vol. 20, no. 14, pp. 15547-15558, 2012.

[176] W. Shi, X. Wang, C. Lin, et al., "Silicon photonic gratingassisted, contra-directional couplers," Opt. Express, vol. 21, no. 3, pp. 3633-3650, 2013.

[177] A. Brimont, D. J. Thomson, F. Y. Gardes, et al., "High-contrast 40 $\mathrm{Gb} / \mathrm{s}$ operation of a $500 \mu \mathrm{m}$ long silicon carrier-depletion slow wave modulator," Opt. Lett., vol. 37, no. 17, Sep 2012, pp. 3504-3506 [Online]. Available at: http://ol.osa.org/ abstract.cfm?URI=ol-37-17-3504.

[178] O. Jafari, W. Shi, and S. LaRochelle, "Silicon photonic modulator using coupled Bragg grating resonators in a MachZehnder structure," in 2019 Conference on Lasers and ElectroOptics (CLEO), San Jose: IEEE, 2019, pp. 1-2. https://doi.org/ 10.1364/CLEO_SI.2019.STh3H.2

[179] S. Hughes, L. Ramunno, J. F. Young, and J. Sipe, “Extrinsic optical scattering loss in photonic crystal waveguides: role of fabrication disorder and photon group velocity," Phys. Rev. Lett., vol. 94, no. 3, p. 033903, 2005

[180] P. Jean, A. Gervais, S. LaRochelle, and W. Shi, "Slow light in subwavelength grating waveguides," IEEE J. Sel. Top. Quant. Electron., vol. 26, no. 2, pp. 1-8, 2019.

[181] J.-H. Han, F. Boeuf, J. Fujikata, S. Takahashi, S. Takagi, and M. Takenaka, "Efficient low-loss InGaAsP/Si hybrid MOS optical modulator," Nat. Photonics, vol. 11, no. 8, p. 486, 2017.

[182] T. Hiraki, T. Aihara, K. Hasebe, et al., "Heterogeneously integrated III-V/Si MOS capacitor Mach-Zehnder modulator," Nat. Photonics, vol. 11, no. 8, pp. 482-485, 2017.

[183] M. He, M. Xu, Y. Ren, et al., "High-performance hybrid silicon and lithium niobate Mach-Zehnder modulators for $100 \mathrm{Gbit} \mathrm{s}^{-1}$ and beyond," Nat. Photonics, vol. 13, no. 5, pp. 359-364, 2019.

[184] H. Zwickel, J. N. Kemal, C. Kieninger, et al., "Electrically packaged silicon-organic hybrid (soh) i/q-modulator for $64 \mathrm{gbd}$ operation," Opt. Express, vol. 26, no. 26, pp. 34580-34591, 2018.

[185] V. Sorianello, M. Midrio, G. Contestabile, et al., "Graphenesilicon phase modulators with gigahertz bandwidth," Nat. Photonics, vol. 12, no. 1, pp. 40-44, 2018.

[186] K. Alexander, J. P. George, J. Verbist, et al., "Nanophotonic pockels modulators on a silicon nitride platform," Nat. Commun., vol. 9, no. 1, p. 3444, 2018.

[187] S. Abel, F. Eltes, J. E. Ortmann, et al., "Large pockels effect in micro-and nanostructured barium titanate integrated on silicon," Nat. Mater., vol. 18, no. 1, pp. 42-47, 2019.

[188] R. Amin, R. Maiti, C. Carfano, et al., " $0.52 \mathrm{v} \mathrm{mm}$ ito-based machzehnder modulator in silicon photonics," APL Photonics, vol. 3, no. 12, p. 126104, 2018. 
[189] B. Zhou, E. Li, Y. Bo, and A. Wang, "High-speed plasmonicsilicon modulator driven by epsilon-near-zero conductive oxide," J. Lightwave Technol., vol. 38, no. 13, pp. 3338-3345, 2020.

[190] O. Marshall, M. Hsu, Z. Wang, B. Kunert, C. Koos, and D. Van Thourhout, "Heterogeneous integration on silicon photonics," Proc. IEEE, vol. 106, no. 12, 2018, pp. 2258-2269.

[191] T. Kobayashi, M. Nakamura, F. Hamaoka, et al., "1-Pb/s (32 SDM $/ 46$ WDM $/ 768 \mathrm{~Gb} / \mathrm{s}$ ) C-band dense SDM transmission over 205.6-km of single-mode heterogeneous multi-core fiber using 96-Gbaud PDM-16QAM channels," in Optical Fiber Communication Conference, Los Angeles: Optical Society of America, 2017, pp. Th5B-1. https://doi.org/10.1364/OFC.2017. Th5B.1.

[192] D. Soma, Y. Wakayama, S. Beppu, et al., “10.16-Peta-B/s dense SDM/WDM transmission over 6-mode 19-core fiber across the C+ L band," J. Lightwave Technol., vol. 36, no. 6, pp. 1362-1368, 2018.

[193] D. Dai and J. E. Bowers, "Silicon-based on-chip multiplexing technologies and devices for peta-bit optical interconnects," Nanophotonics, vol. 3, nos 4-5, pp. 283-311, 2014.

[194] P. Dong, "Silicon photonic integrated circuits for wavelengthdivision multiplexing applications," IEEE J. Sel. Top. Quant. Electron., vol. 22, no. 6, pp. 370-378, 2016.

[195] D. Dai, "Silicon nanophotonic integrated devices for on-chip multiplexing and switching,” J. Lightwave Technol., vol. 35, no. 4, pp. 572-587, 2016.

[196] Y. Tan, H. Wu, and D. Dai, "Silicon-based hybrid (de) multiplexer for wavelength-/polarization-division-multiplexing," J. Lightwave Technol., vol. 36, no. 11, pp. 2051-2058, 2018.

[197] J. Wang, CMOS-compatible Key Engineering Devices for HighSpeed Silicon-Based Optical Interconnections, Springer, 2018.

[198] H. Wu, Y. Tan, and D. Dai, "Ultra-broadband high-performance polarizing beam splitter on silicon," Opt. Express, vol. 25, no. 6 , pp. 6069-6075, 2017.

[199] Y. Tian, J. Qiu, C. Liu, S. Tian, Z. Huang, and J. Wu, “Compact polarization beam splitter with a high extinction ratio over S+ c+ I band," Opt. Express, vol. 27, no. 2, pp. 999-1009, 2019.

[200] H.-C. Chung and S.-Y. Tseng, "Ultrashort and broadband silicon polarization splitter-rotator using fast quasiadiabatic dynamics," Opt. Express, vol. 26, no. 8, pp. 9655-9665, 2018.

[201] Y. Ding, H. Ou, and C. Peucheret, "Wideband polarization splitter and rotator with large fabrication tolerance and simple fabrication process," Opt. Lett., vol. 38, no. 8, pp. 1227-1229, 2013.

[202] W. D. Sacher, T. Barwicz, B. J. Taylor, and J. K. Poon, "Polarization rotator-splitters in standard active silicon photonics platforms," Opt. Express, vol. 22, no. 4, pp. 3777-3786, 2014.

[203] H. Guan, A. Novack, M. Streshinsky, et al., "Cmos-compatible highly efficient polarization splitter and rotator based on a double-etched directional coupler," Opt. Express, vol. 22, no. 3, pp. 2489-2496, 2014

[204] K. Tan, Y. Huang, G.-Q. Lo, C. Yu, and C. Lee, "Ultra-broadband fabrication-tolerant polarization splitter and rotator," in Optical Fiber Communication Conference, Los Angeles: Optical Society of America, 2017, pp. Th1G-7. https://doi.org/10. 1364/OFC.2017.Th1G.7.

[205] Y. Zhang, Q. Zhu, Y. He, and Y. Su, "Silicon polarization splitter and rotator with tolerance to width variations using a nonlinearly-tapered and partially-etched directional coupler," in Optical Fiber Communication Conference, San Diego: Optical Society of America, 2019, pp. W1E-4. https://doi.org/10.1364/ OFC.2019.W1E.4.

[206] H. Xu and Y. Shi, "Ultra-broadband silicon polarization splitterrotator based on the multi-mode waveguide," Opt. Express, vol. 25, no. 15, pp. 18 485-18 491, 2017.

[207] Y. He, Y. Zhang, X. Wang, et al., "Silicon polarization splitter and rotator using a subwavelength grating based directional coupler," in 2017 Optical Fiber Communications Conference and Exhibition (OFC), Los Angeles: IEEE, 2017, pp. 1-3. https://doi. org/10.1364/OFC.2017.Th1G.6.

[208] D. Guo and T. Chu, "Broadband and low-crosstalk polarization splitter-rotator with optimized tapers," OSA Contin., vol. 1, no. 3, pp. 841-850, 2018.

[209] Corporate Headquarters, Introduction to Dwdm Technology, San Jose: Cisco Systems, Inc., 2000.

[210] ITU-T. G.694.1: Spectral grids for WDM applications: DWDM frequency grid, ITU, 2012.

[211] P. Cheben, J. Schmid, A. Delâge, et al., “A high-resolution silicon-on-insulator arrayed waveguide grating microspectrometer with sub-micrometer aperture waveguides," Opt. Express, vol. 15, no. 5, pp. 2299-2306, 2007.

[212] S. Pathak, D. Van Thourhout, and W. Bogaerts, "Design tradeoffs for silicon-on-insulator-based awgs for (de) multiplexer applications,” Opt. Lett., vol. 38, no. 16, pp. 2961-2964, 2013.

[213] S. Cheung, T. Su, K. Okamoto, and S. Yoo, "Ultra-compact silicon photonic $512 \times 51225 \mathrm{ghz}$ arrayed waveguide grating router," IEEE J. Sel. Top. Quant. Electron., vol. 20, no. 4, pp. 310-316, 2013.

[214] M. Gehl, D. Trotter, A. Starbuck, A. Pomerene, A. Lentine, and C. DeRose, "Active phase correction of high resolution silicon photonic arrayed waveguide gratings," Opt. Express, vol. 25, no. 6, pp. 6320-6334, 2017.

[215] X. Pommarede, K. Hassan, P. Billondeau, et al., “ $16 \times 100$ ghz echelle grating-based wavelength multiplexer on silicon-oninsulator platform," IEEE Photonics Technol. Lett., vol. 29, no. 6, pp. 493-495, 2017.

[216] D. Munk, M. Katzman, Y. Kaganovskii, et al., "Eight-channel silicon-photonic wavelength division multiplexer with $17 \mathrm{ghz}$ spacing," IEEE J. Sel. Top. Quant. Electron., vol. 25, no. 5, pp. 1-10, 2019.

[217] R. Boeck, M. Caverley, L. Chrostowski, and N. A. Jaeger, "Experimental demonstration of a silicon-on-insulator highperformance double microring filter using mzi-based coupling," Opt. Lett., vol. 40, no. 2, pp. 276-279, 2015.

[218] S. Bélanger-de Villers, D. Hould, and W. Shi, "Ultra-compact dwdm filter tunable across the c-band," in Optical Fiber Communication Conference, San Diego: Optical Society of America, 2019, pp. W2A-4. https://doi.org/10.1364/OFC.2019. W2A.4.

[219] J. St-Yves, H. Bahrami, P. Jean, S. LaRochelle, and W. Shi, "Widely bandwidth-tunable silicon filter with an unlimited freespectral range," Opt. Lett., vol. 40, no. 23, Dec 2015, pp. 5471-5474 [Online]. Available at: http://ol.osa.org/ abstract.cfm?URI=0l-40-23-5471.

[220] S. Pathak, P. Dumon, D. Van Thourhout, and W. Bogaerts, "Comparison of awgs and echelle gratings for wavelength division multiplexing on silicon-on-insulator," IEEE Photonics J., vol. 6, no. 5, pp. 1-9, 2014. 
[221] W. Bogaerts, S. Pathak, A. Ruocco, and S. Dwivedi, "Silicon photonics non-resonant wavelength filters: comparison between awgs, echelle gratings, and cascaded mach-zehnder filters," in Integrated Optics: Devices, Materials, and Technologies XIX, vol. 9365, International Society for Optics and Photonics, 2015, p. $93650 \mathrm{H}$.

[222] Y. Yang, X. Hu, J. Song, et al., "Thermo-optically tunable silicon awg with above 600 ghz channel tunability," IEEE Photonics Technol. Lett., vol. 27, no. 22, pp. 2351-2354, 2015.

[223] X. Chen, M. M. Milosevic, D. J. Thomson, et al., "Post-fabrication phase trimming of mach-zehnder interferometers by laser annealing of germanium implanted waveguides," Photonics Res., vol. 5, no. 6, pp. 578-582, 2017.

[224] M. M. Milosevic, X. Chen, W. Cao, et al., "Ion implantation in silicon for trimming the operating wavelength of ring resonators," IEEE J. Sel. Top. Quant. Electron., vol. 24, no. 4, pp. 1-7, 2018.

[225] S. Chen, X. Fu, J. Wang, Y. Shi, S. He, and D. Dai, “Compact dense wavelength-division (de) multiplexer utilizing a bidirectional arrayed-waveguide grating integrated with a mach-zehnder interferometer," J. Lightwave Technol., vol. 33, no. 11, pp. 2279-2285, 2015.

[226] K. Takada, M. Abe, T. Shibata, and K. Okamoto, “A. 2.5 ghz-spaced 1080-channel tandem multi/demultiplexer covering the. s-, c-, and l-bands using an arrayed-waveguide grating with Gaussian passbands as a primary filter," IEEE Photonics Technol. Lett., vol. 14, no. 5, pp. 648-650, 2002.

[227] B. Akca, C. Doerr, G. Sengo, K. Wörhoff, M. Pollnau, and R. de Ridder, "Broad-spectral-range synchronized flat-top arrayed-waveguide grating applied in a 225-channel cascaded spectrometer," Opt. Express, vol. 20, no. 16, pp. 18313-18318, 2012.

[228] B. I. Akca and C. R. Doerr, "Interleaved silicon nitride awg spectrometers," IEEE Photonics Technol. Lett., vol. 31, no. 1, pp. 90-93, 2018.

[229] W. Bogaerts, P. De Heyn, T. Van Vaerenbergh, et al., "Silicon microring resonators," Laser Photonics Rev., vol. 6, no. 1, pp. 47-73, 2012

[230] S. Xiao, M. H. Khan, H. Shen, and M. Qi, "A highly compact thirdorder silicon microring add-drop filter with a very large free spectral range, a flat passband and a low delay dispersion," Opt. Express, vol. 15, no. 22, pp. 14 765-14 771, 2007.

[231] P. Dong, A. Melikyan, and K. Kim, "Commercializing silicon microring resonators: technical challenges and potential solutions," in 2018 Conference on Lasers and Electro-Optics (CLEO), San Diego: IEEE, 2018, pp. 1-2.

[232] D.-X. Xu, J. Schmid, G. Reed, et al., "Silicon photonic integration platform-have we found the sweet spot? " IEEE J. Sel. Top. Quant. Electron., vol. 20, no. 4, pp. 189-205, July 2014.

[233] K. Padmaraju and K. Bergman, "Resolving the thermal challenges for silicon microring resonator devices," Nanophotonics, vol. 3, no. 4-5, pp. 269-281, 2014.

[234] H. Jayatilleka, H. Shoman, L. Chrostowski, and S. Shekhar, "Photoconductive heaters enable control of large-scale silicon photonic ring resonator circuits," Optica, vol. 6, no. 1, pp. 84-91, 2019.
[235] H. Jayatilleka, H. Shoman, R. Boeck, N. A. Jaeger, L. Chrostowski, and S. Shekhar, "Automatic configuration and wavelength locking of coupled silicon ring resonators," J. Lightwave Technol., vol. 36, no. 2, pp. 210-218, 2017.

[236] Y. Yu, L. Jin, Y. Lu, Y. Huang, and L. Li, “ $400 \times 377.6 \mathrm{gb} / \mathrm{s} \mathrm{c} \mathrm{+} \mathrm{I}$ band seamless transmission over $200 \mathrm{~km}$ with Raman amplifier using pdm-256qam," in 2018 European Conference on Optical Communication (ECOC), Rome: IEEE, 2018, pp.1-3. https://doi. org/10.1109/ECOC.2018.8535505.

[237] Y. Sasaki, K. Takenaga, K. Aikawa, Y. Miyamoto, and T. Morioka, "Single-mode 37-core fiber with a cladding diameter of $248 \mu \mathrm{m}$," in 2017 Optical Fiber Communications Conference and Exhibition (OFC), Los Angeles: IEEE, 2017, pp. 1-3. https://doi. org/10.1364/OFC.2017.Th1H.2.

[238] R. Ryf, N. K. Fontaine, S. Wittek, et al., "High-spectral-efficiency mode-multiplexed transmission over graded-index multimode fiber," in 2018 European Conference on Optical Communication (ECOC), Rome: IEEE, 2018, pp. 1-3. https://doi.org/10.1109/ ECOC.2018.8535536.

[239] T. Sakamoto, T. Matsui, K. Saitoh, et al., "Low-loss and lowdmd 6-mode 19-core fiber with cladding diameter of less than 250 um," J. Lightwave Technol., vol. 35, no. 3, pp. 443-449, 2016.

[240] T. Sakamoto, K. Saitoh, S. Saitoh, et al., "120 spatial channel few-mode multi-core fibre with relative core multiplicity factor exceeding 100," in 2018 European Conference on Optical Communication (ECOC), Rome: IEEE, 2018, pp. 1-3. https://doi. org/10.1109/ECOC.2018.8535257.

[241] W. Wang, H.-D. Xu, Q.-H. Yang, et al., "Large mode area microstructured fiber supporting 56 super-oam modes," Opt. Express, vol. 27, no. 20, pp. 27991-28008, 2019.

[242] S. Hong, Y. S. Lee, H. Choi, et al., "Hollow silica photonic crystal fiber guiding 101 orbital angular momentum modes without phase distortion in C + L band," J. Lightwave Technol., vol. 38, no. 5, pp. 1010-1018, 2019.

[243] C. Brunet, P. Vaity, Y. Messaddeq, S. LaRochelle, and L. A. Rusch, "Design, fabrication and validation of an oam fiber supporting 36 states," Opt. Express, vol. 22, no. 21, pp. 26117-26127, 2014.

[244] Y. Ding and K. Yvind, "Efficient silicon pic mode multiplexer using grating coupler array with aluminum mirror for few-mode fiber," in 2015 Conference on Lasers and Electro-Optics (CLEO), San Diego: IEEE, 2015, pp.1-2. https://doi.org/10.1364/CLEO_ SI.2015.STh1F.1.

[245] Y. Tong, W. Zhou, X. Wu, and H. K. Tsang, “Efficient mode multiplexer for few-mode fibers using integrated silicon-oninsulator waveguide grating coupler," IEEE J. Quant. Electron., vol. 56, no. 1, pp. 1-7, 2019.

[246] D. Dai and M. Mao, "Mode converter based on an inverse taper for multimode silicon nanophotonic integrated circuits," Opt. Express, vol. 23, no. 22, pp. 28376-28388, 2015.

[247] S. B. Yoo, B. Guan, and R. P. Scott, "Heterogeneous 2d/3d photonic integrated microsystems," Microsyst. Nanoeng., vol. 2, p. 16030, 2016.

[248] N. Lindenmann, G. Balthasar, D. Hillerkuss, et al., "Photonic wire bonding: a novel concept for chip-scale interconnects," Opt. Express, vol. 20, no. 16, pp. 17667-17677, 2012. 
[249] M. Piels, J. F. Bauters, M. L. Davenport, M. J. Heck, and J. E. Bowers, "Low-loss silicon nitride awg demultiplexer heterogeneously integrated with hybrid iii-v/silicon photodetectors," J. Lightwave Technol., vol. 32, no. 4, pp. 817-823, 2013.

[250] S. Gross, N. Riesen, J. D. Love, and M. J. Withford, "C-band mode-selective couplers fabricated by the femtosecond laser direct-write technique," in Optical Fiber Communication Conference, Los Angeles: Optical Society of America, 2015, pp. W3B-2. https://doi.org/10.1364/OFC.2015.W3B.2.

[251] J. Dong, K. S. Chiang, and W. Jin, "Compact three-dimensional polymer waveguide mode multiplexer," J. Lightwave Technol., vol. 33, no. 22, pp. 4580-4588, 2015.

[252] Y. Wu and K. S. Chiang, "Ultra-broadband mode multiplexers based on three-dimensional asymmetric waveguide branches," Opt. Lett., vol. 42, no. 3, pp. 407-410, 2017.

[253] N. Hanzawa, K. Saitoh, T. Sakamoto, et al., "Demonstration of plc-based six-mode multiplexer for mode division multiplexing transmission," in 2015 European Conference on Optical Communication (ECOC), Valencia: IEEE, 2015, pp. 1-3. https:// doi.org/10.1109/ECOC.2015.7341717.

[254] X. Cai, J. Wang, M. J. Strain, et al., "Integrated compact optical vortex beam emitters," Science, vol. 338, no. 6105, pp. 363-366, 2012.

[255] D. Zhang, X. Feng, and Y. Huang, "Encoding and decoding of orbital angular momentum for wireless optical interconnects on chip," Opt. Express, vol. 20, no. 24, pp. 26986-26995, 2012.

[256] G. Rui, B. Gu, Y. Cui, and Q. Zhan, "Detection of orbital angular momentum using a photonic integrated circuit," Sci. Rep., vol. 6, p. 28262, 2016

[257] S. Li, Z. Nong, X. Wu, et al., "Demonstration of chip-to-chip communication based on ultra-compact orbital angular momentum (de) multiplexers," in CLEO: Science and Innovations, San Diego: Optical Society of America, 2018, pp. STu3B-2. https://doi.org/10.1364/CLEO_SI.2018. STu3B.2.

[258] N. Zhou, S. Zheng, X. Cao, et al., "Ultra-compact broadband polarization diversity orbital angular momentum generator with 3.6× $3.6 \mu \mathrm{m} 2$ footprint," Sci. Adv., vol. 5, no. 5, 2019, Art no. eaau9593.

[259] N. K. Fontaine, C. R. Doerr, and L. L. Buhl, "Efficient multiplexing and demultiplexing of free-space orbital angular momentum using photonic integrated circuits," in OFC/NFOEC, Los Angeles: IEEE, 2012, pp. 1-3. https://doi.org/10.1364/OFC. 2012.0Tu1l.2.

[260] Y. Chen, L. A. Rusch, and W. Shi, "Design of an integrated circular-polarized oam generator/multiplexer," in 2016 IEEE Photonics Society Summer Topical Meeting Series (SUM), Newport Beach: IEEE, 2016, pp. 205-206. https://doi.org/10. 1109/PHOSST.2016.7548810.

[261] Y. Chen, L. A. Rusch, and W. Shi, “Integrated circularly polarized oam generator and multiplexer for fiber transmission," IEEE J. Quant. Electron., vol. 54, no. 2, pp. 1-9, 2017.

[262] Y. Chen, Z. Lin, S. Bélanger-de Villers, L. A. Rusch, and W. Shi, "Wdm-compatible polarization-diverse oam generator and multiplexer in silicon photonics," IEEE J. Sel. Top. Quant. Electron., vol. 26, no. 2, pp. 1-7, 2019.

[263] L. A. Rusch, M. Rad, K. Allahverdyan, I. Fazal, and E. Bernier, "Carrying data on the orbital angular momentum of light," IEEE Commun. Mag., vol. 56, no. 2, pp. 219-224, 2018.

[264] J. Sun, A. Yaacobi, M. Moresco, D. Coolbaugh, and M. R. Watts, "Chip-scale continuously tunable optical orbital angular momentum generator," 2014, arXiv preprint arXiv:1408.3315, https://doi.org/10.1364/cleo_at.2014.jth5b.4.

[265] C. Hössbacher, Y. Salamin, Y. Fedoryshyn, et al., “Optical interconnect solution with plasmonic modulator and ge photodetector array," IEEE Photonics Technol. Lett., vol. 29, no. 21, pp. 1760-1763, 2017.

[266] J. Du, L. Zheng, K. Xu, et al., "High speed and small footprint silicon micro-ring modulator assembly for space-divisionmultiplexed 100-gbps optical interconnection," Opt. Express, vol. 26, no. 11, pp. 13 721-13 729, 2018.

[267] P. De Heyn, V. I. Kopp, S. A. Srinivasan, et al., "Ultra-dense $16 \times 56 \mathrm{gb} / \mathrm{s}$ nrz gesi eam-pd arrays coupled to multicore fiber for short-reach $896 \mathrm{gb} / \mathrm{s}$ optical links," in Optical Fiber Communication Conference, Los Angeles: Optical Society of America, 2017, pp. Th1B-7. https://doi.org/10.1364/OFC.2017. Th1B.7.

[268] N. Riesen, S. Gross, J. D. Love, Y. Sasaki, and M. J. Withford, "Monolithic mode-selective few-mode multicore fiber multiplexers," Sci. Rep., vol. 7, no. 1, pp. 1-9, 2017.

[269] S. Chen, W. Li, J. Wu, et al., "Electrically pumped continuouswave iii-v quantum dot lasers on silicon," Nat. Photonics, vol. 10, no. 5, p. 307, 2016.

[270] T. J. Kippenberg, R. Holzwarth, and S. A. Diddams, "Microresonator-based optical frequency combs," Science, vol. 332, no. 6029, pp. 555-559, 2011.

[271] P. Marin-Palomo, J. N. Kemal, M. Karpov, et al., "Microresonator-based solitons for massively parallel coherent optical communications," Nature, vol. 546, no. 7657, pp. 274-279, 2017.

[272] B. Stern, X. Ji, Y. Okawachi, A. L. Gaeta, and M. Lipson, "Batteryoperated integrated frequency comb generator," Nature, vol. 562, no. 7727, pp. 401-405, 2018.

[273] K. Suzuki, R. Konoike, J. Hasegawa, et al., "Low-insertion-loss and power-efficient $32 \times 32$ silicon photonics switch with extremely high-ठ silica plc connector," J. Lightwave Technol., vol. 37, no. 1, pp. 116-122, 2019.

[274] P. Dumais, D. J. Goodwill, D. Celo, et al., "Silicon photonic switch subsystem with 900 monolithically integrated calibration photodiodes and 64-fiber package," J. Lightwave Technol., vol. 36, no. 2, pp. 233-238, 2017.

[275] T. J. Seok, K. Kwon, J. Henriksson, J. Luo, and M. C. Wu, "Waferscale silicon photonic switches beyond die size limit," Optica, vol. 6, no. 4, pp. 490-494, 2019.

[276] N. Dupuis, J. E. Proesel, N. Boyer, et al., “An $8 \times 8$ silicon photonic switch module with nanosecond-scale reconfigurability," in 2020 Optical Fiber Communications Conference and Exhibition (OFC), San Diego: IEEE, 2020, pp. Th4A.6. https://doi.org/10.1364/OFC.2020.Th4A.6. 